\title{
Integrable, oblique travelling waves in quasi-charge-neutral two-fluid plasmas
}

\author{
G. M. Webb ${ }^{1}$, C. M. Ko ${ }^{1,2, *}$, R. L. Mace ${ }^{3}$, J. F. McKenzie ${ }^{1,3}$, and G. P. Zank ${ }^{1}$ \\ ${ }^{1}$ Institute of Geophysics and Planetary Physics, University of California Riverside, Riverside CA 92521, USA \\ ${ }^{2}$ Institute of Astronomy, Department of Physics and Centre for Complex Systems, National Central University, Chung-Li, \\ 32054, Taiwan, Republic of China \\ ${ }^{3}$ School of Physics, University of KwaZulu-Natal, Westville Campus, Private Bag X54001, Durban 4000, South Africa \\ * work carried out on leave at: IGPP, University of California Riverside, CA, USA
}

Received: 13 November 2007 - Revised: 9 January 2008 - Accepted: 9 January 2008 - Published: 25 February 2008

\begin{abstract}
A Hamiltonian description of oblique travelling waves in a two-fluid, charge-neutral, electron-proton plasma reveals that the transverse momentum equations for the electron and proton fluids are exactly integrable in cases where the total transverse momentum flux integrals, $P_{y}^{(d)}$ and $P_{z}^{(d)}$, are both zero in the de Hoffman Teller (dHT) frame. In this frame, the transverse electric fields are zero, which simplifies the transverse momentum equations for the two fluids. The integrable travelling waves for the case $P_{y}^{(d)}=P_{z}^{(d)}=0$, are investigated based on the Hamiltonian trajectories in phase space, and also on the longitudinal structure equation for the common longitudinal fluid velocity component $u_{x}$ of the electron and proton fluids. Numerical examples of a variety of travelling waves in a cold plasma, including oscillitons, are used to illustrate the physics. The transverse, electron and proton velocity components $u_{j y}$ and $u_{j z}(j=e, p)$ of the waves exhibit complex, rosette type patterns over several periods for $u_{x}$. The role of separatrices in the phase space, the rotational integral and the longitudinal structure equation on the different wave forms are discussed.
\end{abstract}

\section{Introduction}

In a recent paper (Webb et al., 2007, hereinafter referred to as paper I), we developed a dual variational principle for nonlinear travelling waves in a charge neutral, non-relativistic, electron-proton plasma. It was shown that travelling waves in this multi-fluid plasma system could be described by two different, but equivalent Hamiltonian formulations. In the first formulation, the Hamiltonian is identified with the total conserved longitudinal $x$-momentum integral of the system, $P_{x}$, in which the energy flux integral $\varepsilon=$ const. is a constraint, and for which $d / d x$ is the Hamiltonian evolution operator. In the

Correspondence to: G. M. Webb

(gmwebb@ucr.edu) second Hamiltonian formulation, the Hamiltonian is the energy flux integral $\varepsilon$, and the $x$-momentum integral $P_{x}=$ const. is a constraint. In the latter formulation the Hamiltonian evolution operator is the advective or Lagrangian time derivative $d / d \tau=u_{x} d / d x$. These dual variational principles are analogous to the dual or multi-symplectic variational principles obtained by Bridges (1992) in a study of travelling water waves. Related work on a Hamiltonian formulation of parallel propagating whistler waves in multi-fluid plasmas has been investigated by Webb et al. (2005), whereas McKenzie, Mace and Doyle (2007) show that the spatial evolution equations for solitary travelling waves in Hall current plasmas can be cast in a Hamiltonian form in which the energy flux integral $\varepsilon$ is the Hamiltonian and the longitudinal momentum integral $P_{x}=$ const. acts as a constraint. The multi-symplectic variational principles in general do not imply integrability of the equations, that are obtained for bi-Hamiltonian systems such as the KdV equation and the nonlinear Schrödinger equation (e.g. Magri, 1978), which possess an infinite number of conservation laws and Lie symmetries.

In a multi-symplectic Hamiltonian system both space and time variables can be used as Hamiltonian evolution variables, and the evolution of both the space and time variables are associated with skew symmetric matrices (see e.g. Bridges, 1997a,b, Hydon, 2005, and Cotter, Holm and Hydon, 2007 for further details). The conditions that are required for integrability is a subject of ongoing debate (e.g. Zakharov, 1991). For ordinary differential equation systems, integrability means that the number of known integrals matches exactly the number of integration constants. For nonlinear differential equations it was conjectured (Ramani et al., 1982), that integrability can be related to whether the equations possess the Painlevé property. The definition of the Painlevé property for ordinary differential equations, is that the only movable singularities its solution can exhibit are poles (see e.g. Steeb and Euler, 1988). For Hamiltonian systems, integrability is associated with the existence

Published by Copernicus Publications on behalf of the European Geosciences Union and the American Geophysical Union. 
of closed orbits in the phase-space, which in turn are associated with integrals and Lie symmetries of the equations. If the system does not have a complete set of integrals, it is non-integrable and the phase space trajectories may be chaotic. It turns out that the system of ordinary differential equations governing the travelling waves in the quasi-neutral, two-fluid plasma system is integrable if the integration constants $P_{y}^{(d)}=0$ and $P_{z}^{(d)}=0$, where $P_{y}^{(d)}$ and $P_{z}^{(d)}$ are the total transverse momentum integration constants for the system in the dHT frame. In this case, the transverse electron and proton momentum differential equations admit an extra integral, the so-called rotational integral, which allows the equations to be completely integrated by quadrature over the longitudinal flow velocity $u_{x}$. In the cases where $P_{y}^{(d)} \neq 0$ and/or $P_{z}^{(d)} \neq 0$, there is no rotational integral, and if there are no other integrals, the system is non-integrable, and may exhibit chaotic trajectories.

The emphasis in the present paper is on travelling wave solutions of the two-fluid equations, mainly because these are the simplest possible solutions. The solutions are a function of one variable, the travelling wave variable $x^{(w)}=x-w t$ (in our analysis we take $w=-U$ ), where $w$ is the constant velocity of the wave. Such waves can be generated in a steady flow past a stationary object (e.g. such as the magnetosphere in the solar wind flow past the Earth). These standing waves in the frame of the object are analogous to the bow wave of a ship, or stationary wave patterns generated by a rock in a stream. Other wave solution forms are in general possible, but will not be investigated in the present paper.

In Webb et al. (2007), the integration constants for the system of differential equations and conservation laws were specified at a fixed point $x=x_{0}$ in the travelling wave frame, or equivalently in the de Hoffman Teller (dHT) frame. It was found that if the total, transverse momentum flux integral in the dHT frame $P_{+0}^{(d)}=P_{y 0}^{(d)}+i P_{z 0}^{(d)}$ is zero, then the system is exactly integrable (the superscript $d$ denotes the dHT frame). The total, transverse momentum flux was specified by the parameter $\delta_{0} \equiv-M_{A e}^{2} \sec \theta P_{+0}^{(d)}$, where $M_{A e}=U / V_{A e}$ is the electron Alfvén Mach number of the wave, $U$ is the travelling wave speed, $V_{A e}$ is the electron fluid Alfvén speed and $\theta$ is the angle between the propagation direction (the $x$-axis) and the reference magnetic field $\mathbf{B}_{0}$ at $\mathbf{x}_{0}$. Because of the assumption of charge neutrality, the number densities of the electron and proton fluids $n_{p}=n_{e}=n$ throughout the wave, and the two fluids have a common longitudinal fluid velocity $u_{x}$ in the travelling wave frame. The parameter $\delta_{0}$ can in general, be written in the form:

$$
\begin{aligned}
\delta_{0}= & -\frac{M_{\mathrm{Ae} 0}^{2}}{u_{x 0} \cos \theta}\left(u_{e y 0}+\mu u_{p y 0}\right. \\
& \left.+i\left[u_{e z 0}+\mu u_{p z 0}-\frac{u_{x 0} \sin \theta \cos \theta}{M_{\mathrm{Ae} 0}^{2}}\right]\right),
\end{aligned}
$$

where $u_{\mathrm{jy} 0}$ and $u_{\mathrm{jz} 0}(j=e, p)$ refer to the transverse electron and proton fluid velocities in the dHT frame, and $M_{\mathrm{Ae} 0}=u_{x 0} / V_{A e}$ is the electron Alfvén Mach number of the flow at $x=x_{0}$. In Eq. (1), all fluid velocities are normalized to the travelling wave speed and $\mu=m_{p} / m_{e}$ is the ratio of the proton and electron masses $m_{p}$ and $m_{e}$.

The Hamiltonian equations describing parallel propagating waves $(\theta=0)$ in a cold plasma, in which $u_{\mathrm{jy} 0}=0$ and $u_{\mathrm{jz} 0}=0(j=e, p)$ as $x_{0} \rightarrow-\infty$ were studied by Webb et al. (2005). They found that the waves were either (i) periodic waves at Mach numbers $0<M<1 / 2$, (ii) oscillitons if $1 / 2<M<1 / \sqrt{2}$, and (iii) periodic waves for $M>1 / \sqrt{2}$, where $M$ is the Alfvén Mach number of the wave based on half the harmonic mean of the electron and proton masses (i.e., the mean particle mass $\bar{m}=m_{p} m_{e} /\left(m_{e}+m_{p}\right)$, where $m_{e}$ and $m_{p}$ denote the electron and proton masses: note that $M \approx M_{\mathrm{Ae} 0}$ as $m_{e} / m_{p}<<1$, where $M_{\mathrm{Ae} 0}$ is the electron Alfvén Mach number). The analysis of Webb et al. (2005) was based in part on the earlier analyses of Sauer et al. (2001, 2002, 2003), Dubinin et al. (2003), and McKenzie et al. (2004). In this paper, we study the class of oblique, integrable, travelling waves, with $\theta \neq 0$, satisfying the condition $\delta_{0}=0$.

Mace et al. (2007) have derived the conservation laws underlying the analysis of McKenzie et al. (2004) for travelling waves in multi-fluid plasmas, by using the frozen in generalized vorticity for barotropic flows for each species. This approach provides a direct and elegant derivation of conservation laws for both multi-dimensional flows and one dimensional flows.

Dubinin et al. (2007) have analyzed the spectrograms of whistler emissions observed by the four CLUSTER spacecraft at $R \sim 14-16 R_{E}$ in the northern dusk magnetosphere in December 2001, at frequencies of $f \sim 20-100 \mathrm{~Hz}$. The wavelet spectrograms of the data suggests that the emissions can be explained by nonlinear, travelling whistler waves of the type investigated in the present paper. For these waves, the protons and the electrons have comparable Reynolds stresses which are mediated by the Maxwell magnetic stresses. Parallel propagating whistlers are shown to undergo nonlinear resonant amplification at one half the electron gyrofrequency, where the phase speed of the wave has a maximum of $V_{A e} / 2\left(V_{A e}\right.$ is the Alfvén speed based on the electron fluid), where the group velocity of the wave matches the phase speed (the group velocity $V_{g}$ exceeds the phase speed of the waves at frequencies $\omega<\Omega_{e}$ ).

In principle, the dual Hamiltonian structure of the travelling waves investigated by Webb et al. $(2005,2007)$ could be related to the general, Hamiltonian, Poisson bracket description of multi-fluid plasmas developed in a more general context by Spencer (1982), Spencer and Kaufman (1982), Holm and Kupershmidt (1983) Sahraoui, Belmont and Rezeau (2003) and Elsässer (1994). Zakharov (1971) developed a Clebsch variable, Hamiltonian description of a magnetized electron plasma, with stationary ions, including the effects 
of charge separation electric fields. This model was used to study nonlinear, three wave resonant interactions. Sahraoui, Belmont and Rezeau (2003) develop a Hamiltonian canonical formalism for two-fluid plasmas, in which the displacement current is neglected, but electron inertia still plays a role and show how this system is related to Hall MHD, when the electron mass is neglected.

The main aim of the present paper is to investigate the nature of the integrable travelling waves for which $\delta_{0}=0$ in Eq. (1). We use the same model as in paper I. The analysis is carried out in the de Hoffman Teller (dHT) frame of MHD shock theory, which is different than the travelling wave frame used by Dubinin et al. (2003). We restrict our analysis to cases where the transformation speed between the travelling wave frame and the $\mathrm{dHT}$ frame, $U \tan \theta<c$, where $U$ is the travelling wave speed and $c$ is the speed of light. The analysis is also restricted to cases where the electron Alfvén speed $V_{A e}<<c$, for which the displacement current in Maxwell's equations can be neglected, and charge neutrality is a good approximation (see Verheest et al., 2004; McKenzie, Dubinin and Sauer, 2005; Webb et al., 2007 for extensive discussion of this very important point).

The basic travelling wave model is outlined in Sect. 2. In Sect. 3, we discuss the action principle and the first Hamiltonian formulation in which the Hamiltonian $H$ is identified with the longitudinal $x$-momentum integral $P_{x}$ and in which the energy integral $\varepsilon=\varepsilon_{0}=$ const. acts as a constraint. In Sect. 4, Hamilton's equations are expressed in terms of the Poisson bracket. It is shown that in the integrable cases $\left(\delta_{0}=0\right)$, a reduction of the phase space from a four dimensional phase space to a two dimensional phase space is possible by exploiting the integrals of the system. The integrability condition $\delta_{0}=0$, further restricts the solutions of interest to a particular Hamiltonian contour in the phase space. The reduction of the phase space from a four to a two dimensional phase space is an example of Hamiltonian reduction (e.g. Marsden and Ratiu, 1994, Olver, 1993. Section 5 presents representative examples of oblique travelling waves for the integrable case $\delta_{0}=0$ in a cold electron-proton plasma. Sect. 6 concludes with a summary and discussion.

\section{The model}

As in paper I, we use a multi-fluid, charge neutral, electronproton model, in which all physical quantities depend only on the position coordinate $X=x+U t$ in the travelling wave frame, where $U$ is the speed of the wave in the lab. frame. We use the dimensionless physical variables:

$$
\bar{x}=\frac{X}{L_{e}}, \quad \overline{\mathbf{B}}=\frac{\mathbf{B}}{B_{0}}, \quad \overline{\mathbf{u}}_{j}=\frac{\mathbf{u}_{j}}{U}, \quad \bar{n}_{j}=\frac{n_{j}}{n_{0}}, \quad j=e, p,
$$

where $B_{0}$ and $n_{0}$ are the constant values of the magnetic field and the number density of the electrons at the fidu- cial point $X=X_{0}$ where the magnetic field $\mathbf{B}$ has the form $\mathbf{B}_{0}=B_{0}(\cos \theta, 0, \sin \theta)^{t}$. We specify the integration constants for the system of conservation laws and differential equations at $X=X_{0}$. The $\mathbf{u}_{j}$ denote the fluid velocity of species $j$. We use the physical parameters:

$$
\begin{aligned}
& L_{e}=\frac{U}{\Omega_{e}}, \quad \Omega_{e}=\frac{e B_{0}}{m_{e}}, \quad \mu=\frac{m_{p}}{m_{e}}, \\
& M_{A e}^{2}=\frac{U^{2}}{V_{A e}^{2}}, \quad V_{A e}^{2}=\frac{B_{0}^{2}}{\mu_{0} m_{e} n_{e}}, \\
& M_{j}^{2}=\frac{U^{2}}{c_{j 0}^{2}}, \quad c_{j 0}^{2}=\frac{\gamma_{j} p_{j 0}}{n_{0} m_{j}}, \quad j=e, p,
\end{aligned}
$$

to characterize the travelling wave. Here, $\Omega_{e}$ is the electron cyclotron frequency, $V_{A e}$ is the Alfvén speed based on the electron number density $n_{e}$, and $M_{A e}$ is the Alfvén Mach number of the wave based on the electron fluid; $\mu$ is the ratio of the proton and electron masses $(\mu=1836) ; M_{j}$ are sonic Mach numbers of the travelling wave, based on the sound speed $c_{j 0}$ of the different plasma species (here $j=e, p$ ), and $L_{e}=U / \Omega_{e}$ is the characteristic scale length for the wave associated with the electron fluid and $\Omega_{e}$ is the electron gyrofrequency. For a charge neutral plasma $n_{e}=n_{p}=n$.

The electron and proton fluids are assumed to have polytropic equations of state of the form:

$p_{j}=p_{j 0}\left(\frac{n_{j}}{n_{0}}\right)^{\gamma_{j}}, \quad j=e, p$,

where $\gamma_{e}$ and $\gamma_{p}$ are the polytropic indices of the electron and proton fluids.

The basic equations for the system consist of the momentum and energy equations, and the number density continuity equations for each species, and the overall momentum and energy equations for the system, coupled with Maxwell's equations. We use a Galilean transformation of the transverse velocities for the two species, relative to that used by Dubinin et al. (2003) and McKenzie et al. (2004) of the form:

$u_{j y}=u_{j y}^{w}, \quad u_{j z}=u_{j z}^{w}+\tan \theta, \quad j=e, p$.

The $\mathbf{u}_{j}^{w}(j=e, p)$ are the velocities in the travelling wave frame used by Dubinin et al. (2003), normalized to the travelling wave speed $U$. The transformation Eq. (5), is from the travelling wave frame to the de-Hoffman Teller (dHT) frame, used in the theory of MHD shocks (de Hoffman and Teller, 1950, Drury, 1983, Webb, Axford and Terasawa, 1983). We assume that the de Hoffman Teller speed $U \tan \theta<c$.

In the further development, (in an abuse of mathematical notation) we omit the over-bar notation for normalized quantitities, unless stated otherwise.

In terms of the variables Eq. (5), the transverse electron and proton momentum equations for the system may be written in the form: 


$$
\begin{aligned}
E_{1}= & \frac{d u_{e}^{+}}{d x}-i \frac{u_{e}^{+} \cos \theta}{u_{x}} \\
& +i\left(\frac{M_{A e}^{2} u_{x 0}}{\cos \theta}\left(u_{e}^{+}+\mu u_{p}^{+}\right)+\delta_{0}\right)=0, \\
E_{2}= & \frac{d u_{e}^{-}}{d x}+i \frac{u_{e}^{-} \cos \theta}{u_{x}} \\
& -i\left(\frac{M_{A e}^{2} u_{x 0}}{\cos \theta}\left(u_{e}^{-}+\mu u_{p}^{-}\right)+\delta_{0}^{*}\right)=0, \\
E_{3}= & \frac{d u_{p}^{+}}{d x}+i \frac{u_{p}^{+} \cos \theta}{\mu u_{x}} \\
& -\frac{i}{\mu}\left(\frac{M_{A e}^{2} u_{x 0}}{\cos \theta}\left(u_{e}^{+}+\mu u_{p}^{+}\right)+\delta_{0}\right)=0, \\
E_{4}= & \frac{d u_{p}^{-}}{d x}-i \frac{u_{p}^{-} \cos \theta}{\mu u_{x}} \\
& +\frac{i}{\mu}\left[\frac{M_{A e}^{2} u_{x 0}}{\cos \theta}\left(u_{e}^{-}+\mu u_{p}^{-}\right)+\delta_{0}^{*}\right]=0 .
\end{aligned}
$$

where

$u_{j}^{ \pm}=u_{j y} \pm i u_{j z}, \quad j=e, p$,

are the complex transverse velocities of the electrons and protons. We note, for later reference, that:

$M_{A}^{2}=(1+\mu) M_{A e}^{2} \sec ^{2} \theta \equiv \frac{U^{2}}{V_{A}^{2} \cos ^{2} \theta}$,

is the square of the total Alfvén Mach number $M_{A}$, based on the total plasma density $\rho=m_{e} n_{e}+m_{p} n_{p} \equiv m_{e}(1+\mu) n$, and the Alfvén phase velocity $V_{A n}=V_{A} \cos \theta$. In Eq. (6) $u_{x}$ is the common, normalized $x$-component of the velocity of the proton and electron fluids and $x$ refers to the normalized position coordinate in the travelling wave frame (i.e. to $\bar{x}$ of Eq. 2). The parameter $\delta_{0}$ in the above equations is given by:

$$
\begin{aligned}
\delta_{0} & =-\frac{M_{A e}^{2}}{\cos \theta} P_{+}^{(d)} \\
& \equiv-\frac{M_{A e}^{2}}{\cos \theta}\left(P_{+}^{(w)}+i u_{x 0}(1+\mu) \tan \theta\right),
\end{aligned}
$$

where $P_{+}^{(d)}=P_{y}^{(d)}+i P_{z}^{(d)}$ is the total, complex, transverse, momentum integral in the dHT frame. It may be expressed in the form:

$$
\begin{aligned}
\delta_{0}= & -\frac{M_{A e}^{2}}{\cos \theta} u_{x 0}\left(u_{e y 0}^{w}+\mu u_{p y 0}^{w}\right) \\
& +i \sin \theta\left[1-u_{x 0} M_{A}^{2}\left(1+\frac{\cot \theta}{\mu+1}\left(u_{e z 0}^{w}+\mu u_{p z 0}^{w}\right)\right)\right] .
\end{aligned}
$$

If $\delta_{0}=0$, the transverse momentum Eqs. (6), are invariant under rotations about the $x$ axis, and admit an extra integral due to this Lie symmetry, and the equations are exactly integrable. The condition $\delta_{0}=0$ is equivalent to the conditon that the total transverse momentum integral in the dHT frame is zero.

We use the dHT frame fluid velocities in the transverse momentum Eq. (6) whereas Dubinin et al. (2003) use the travelling wave frame velocities. The Dubinin et al. (2003) Eqs. (57) are equivalent to Eq. (6) in the special case where

$\delta_{0}=i \sin \theta\left(1-M_{A}^{2} u_{x 0}\right), \quad u_{j 0}^{w+}=0, \quad j=e, p$,

and $u_{x 0}=1$. Note that $\delta_{0}=0$ is satisfied for the case of parallel propagation $(\theta=0)$ and for $u_{e y 0}^{w}=u_{p y 0}^{w}=0$. Webb et al. (2005), studied this integrable case, and obtained compact travelling waves, whistler oscillitons and periodic travelling wave solutions. If $\theta \neq 0$ then the equations are integrable if $\delta_{0}=0$ i.e., if $M_{A}^{2}=1 / u_{x 0}$ in Eq. (11). More generally, $\delta_{0}$ may be expressed in the form:

$\delta_{0}=-\frac{M_{\mathrm{Ae} 0}^{2}}{u_{x 0} \cos \theta}\left[u_{e y 0}+\mu u_{p y 0}+i\left(u_{e z 0}+\mu u_{p z 0}-b\right)\right]$,

where

$b=\frac{u_{x 0} \sin \theta \cos \theta}{M_{\mathrm{Ae} 0}^{2}} \equiv \frac{(\mu+1) \tan \theta}{M_{A}^{2} u_{x 0}}, \quad M_{\mathrm{Ae} 0}^{2}=\frac{u_{x 0}^{2}}{V_{A e}^{2}}$.

In Eq. (12) and Eq. (13) $M_{\mathrm{Ae} 0}^{2}=u_{x 0}^{2} / V_{A e}^{2}$ is the electron, Alfvén Mach number of the flow. Thus, the integrability condition $\delta_{0}=0$ requires:

$u_{e y 0}+\mu u_{p y 0}=0, \quad$ and $\quad u_{e z 0}+\mu u_{p z 0}=b$.

The expression Eq. (12) for $\delta_{0}$ is the same as that in Eq. (1). The conditions Eq. (14), which are equivalent to $\delta_{0}=0$, can be solved for the transverse electron fluid velocity $\left(0, u_{e y 0}, u_{e z 0}\right)$ in terms of the transverse proton velocity $\left(0, u_{p y 0}, u_{p z 0}\right)$ and vice-versa. Conditions Eq. (14) are central to the study of the integrable travelling wave solutions studied in this paper.

The total transverse momentum equations for the electrons and the protons can be solved for the complex transverse magnetic field $B^{+}=\left(B_{y}+i B_{z}\right) / B_{0}$ in the wave as:

$B^{+}=\frac{M_{A e}^{2}}{\cos \theta} u_{x 0}\left(u_{e}^{+}+\mu u_{p}^{+}\right)+\delta_{0}$,

where the $u_{j}^{+}(j=e, p)$ are the fluid velocities in the de Hoffman Teller frame (cf. 7).

Dubinin et al. (2003) assumed that $u_{j}^{w}=\left|u_{j}^{ \pm w}\right|(j=e, p)$ vanish and $\mathbf{B} \rightarrow B_{0}(\cos \theta, 0, \sin \theta)^{t}$ as $x \rightarrow-\infty$. The boundary conditions $u_{j}^{w} \rightarrow 0$ correspond to the boundary conditions $u_{j} \equiv\left|u_{j}^{ \pm}\right| \rightarrow \tan \theta$ as $|x| \rightarrow \infty$ in the dHT frame. 
The longitudinal momentum equation for the system may be written in the form (Webb et al., 2007):

$P_{x}^{(w)}=P+P_{B}=P_{x 0}^{(w)}$,

where

$P=\frac{\rho u_{x}^{2}+p_{e}+p_{p}}{\rho_{0} U^{2}}, \quad P_{B}=\frac{B_{\perp}^{2}}{2 \mu_{0} \rho_{0} U^{2}}$,

are the normalized fluid dynamical and magnetic pressure contributions repectively to the total longitudinal momentum flux, $\rho=\left(m_{e}+m_{p}\right) n$ is the total plasma and $u_{x}$ in Eq. (17) is the non-normalized longitudinal flow speed of the electron and proton fluids. Here, $P$ and $P_{B}$ can be expressed in the form:

$$
\begin{aligned}
P\left(\hat{u}_{x}\right) & =u_{x 0}^{2}\left[\hat{u}_{x}+\frac{1}{\mu+1}\left(\frac{\hat{u}_{x}^{-\gamma_{e}}}{\gamma_{e} M_{e 0}^{2}}+\frac{\mu \hat{u}_{x}^{-\gamma_{p}}}{\gamma_{p} M_{p 0}^{2}}\right)\right], \\
P_{B} & =\frac{u_{x 0}^{2}}{2(\mu+1) M_{\mathrm{Ae} 0}^{2}}\left|\frac{M_{\mathrm{Ae} 0}^{2}}{\cos \theta}\left(\hat{u}_{e}^{+}+\mu \hat{u}_{p}^{+}\right)+\delta_{0}\right|^{2},
\end{aligned}
$$

where

$\hat{u}_{x}=\frac{u_{x}}{u_{x 0}}, \quad \hat{u}_{j}^{ \pm}=\frac{u_{j}^{ \pm}}{u_{x 0}}, \quad M_{j 0}^{2}=\frac{u_{x 0}^{2}}{c_{j 0}^{2}}, \quad M_{\mathrm{Ae} 0}^{2}=\frac{u_{x 0}^{2}}{V_{A e}^{2}}$,

$(j=e, p)$ are the normalized fluid velocities and Mach numbers based on the longitudinal flow speed $u_{x 0}$ at $x=x_{0}$. Note that

$$
M_{j 0}^{2}=u_{x 0}^{2} M_{j}^{2}, \quad M_{A j 0}^{2}=u_{x 0}^{2} M_{A j}^{2}, \quad \hat{u}_{j}=\frac{u_{j}}{u_{x 0}}, \quad j=e, p .
$$

The Mach numbers $M_{j 0}$ and $M_{A j 0}$ based on $u_{x 0}$ are more physically relevant than the Mach numbers $M_{j}$ and $M_{A j}$ based on the travelling wave speed $U$. However, both normalizations are useful in describing the system. Using the normalized variables Eq. (20), the energy integral for the system may be written in the form:

$$
\begin{aligned}
\varepsilon= & u_{x 0}^{2}\left[\frac{1}{2}(\mu+1) \hat{u}_{x}^{2}+\frac{\hat{u}_{x}^{1-\gamma_{e}}}{\left(\gamma_{e}-1\right) M_{e 0}^{2}}+\frac{\mu \hat{u}_{x}^{1-\gamma_{p}}}{\left(\gamma_{p}-1\right) M_{p 0}^{2}}\right. \\
& \left.+\frac{1}{2}\left(\hat{u}_{e}^{2}+\mu \hat{u}_{p}^{2}\right)-\frac{1}{2}(\mu+1) \hat{V}_{H T}^{2}\right]=\varepsilon_{0},
\end{aligned}
$$

where $\hat{V}_{H T}=U \tan \theta / u_{x 0}$ is the normalized transformation speed between the travelling wave frame and the de-Hoffman Teller frame.

\subsection{Amplitude and phase equations}

In this section, we list the amplitude and phase form of the transverse electron and proton momentum Eq. (6) in which $u_{j}^{ \pm}=u_{j} \exp \left( \pm i \phi_{j}\right), j=e, p$. The equations may be written in the form:

$$
\begin{aligned}
\frac{d u_{e}}{d x}= & \frac{M_{A e}^{2} u_{x 0}}{\cos \theta} \mu u_{p} \sin \phi-\operatorname{Re}\left[i \delta_{0} \exp \left(-i \phi_{e}\right)\right], \\
\frac{d \phi_{e}}{d x}= & -\frac{M_{A e}^{2} u_{x 0}}{\cos \theta}\left(1+\frac{\mu u_{p}}{u_{e}} \cos \phi\right)+\frac{\cos \theta}{u_{x}} \\
& -\frac{1}{u_{e}} \operatorname{Im}\left[i \delta_{0} \exp \left(-i \phi_{e}\right)\right], \\
\frac{d u_{p}}{d x}= & \frac{M_{A e}^{2} u_{x 0}}{\mu \cos \theta} u_{e} \sin \phi+\frac{1}{\mu} \operatorname{Re}\left[i \delta_{0} \exp \left(-i \phi_{p}\right)\right], \\
\frac{d \phi_{p}}{d x}= & \frac{M_{A e}^{2} u_{x 0}}{\cos \theta}\left(1+\frac{u_{e}}{\mu u_{p}} \cos \phi\right)-\frac{\cos \theta}{\mu u_{x}} \\
& +\frac{1}{\mu u_{p}} \operatorname{Im}\left[i \delta_{0} \exp \left(-i \phi_{p}\right)\right] .
\end{aligned}
$$

From Eqs. (23-26) we obtain auxiliary equations for $\phi=\phi_{p}$ $\phi_{e}$ and $\tilde{\phi}=\phi_{p}+\phi_{e}$ as:

$$
\begin{aligned}
\frac{d \phi}{d x}= & \frac{M_{A e}^{2} u_{x 0}}{\cos \theta}\left[2+\left(\frac{u_{e}}{\mu u_{p}}+\frac{\mu u_{p}}{u_{e}}\right) \cos \phi\right] \\
& -\frac{(\mu+1) \cos \theta}{\mu u_{x}} \\
& +\operatorname{Im}\left[i \delta_{0}\left(\frac{\exp \left(-i \phi_{p}\right)}{\mu u_{p}}+\frac{\exp \left(-i \phi_{e}\right)}{u_{e}}\right)\right], \\
\frac{d \tilde{\phi}}{d x}= & \frac{M_{A e}^{2} u_{x 0}}{\cos \theta}\left(\frac{u_{e}}{\mu u_{p}}-\frac{\mu u_{p}}{u_{e}}\right) \cos \phi+\frac{(\mu-1) \cos \theta}{\mu u_{x}} \\
& +\operatorname{Im}\left[i \delta_{0}\left(\frac{\exp \left(-i \phi_{p}\right)}{\mu u_{p}}-\frac{\exp \left(-i \phi_{e}\right)}{u_{e}}\right)\right] .
\end{aligned}
$$

\section{Variational and Hamiltonian formulation}

In this section we provide a brief synopsis of the first variational principle of paper I and the corresponding Hamiltonian formulation of the transverse momentum Eqs. (6) for the electron and proton fluids. These results are used in Sects. 4 and 5 to investigate the class of integrable waves with $\delta_{0}=0$.

The transverse momentum equations for the electron and proton fluids Eq. (6) can be combined to give the equation:

$$
\frac{d}{d x}\left(u_{e}^{2}-\mu^{2} u_{p}^{2}\right)=2 \operatorname{Im}\left[\delta_{0}\left(u_{e}^{-}+\mu u_{p}^{-}\right)\right] .
$$

Thus, if $\delta_{0}=0$, we obtain the integral:

$\mathcal{R}=u_{e}^{2}-\mu^{2} u_{p}^{2}=$ const.

The integral Eq. (30) was also derived by Dubinin et al. (2003), McKenzie et al. (2004) and Webb et al. (2005) for the case of parallel propagating waves for $\theta=0$ and $u_{j y 0}^{w}=u_{j y 0}^{w}=0$ as $x_{0} \rightarrow-\infty$. The integral Eq. (30) also applies for oblique, travelling waves with $\delta_{0}=0$. The integral Eq. (30) is related to invariance of the transverse momentum 
equations and the travelling wave system under rotation of the $y$ and $z$ components of the transverse fields. We show in Sect. 4.1 how this Lie symmetry and the space translation symmetry allows a reduction of the system for $\delta_{0}=0$ to a completely integrable system in which the Hamiltonian dynamics takes place on a two-dimensional reduced phase space (it turns out that the $\delta_{0}=0$ condition restricts the dynamics to a particular contour of the Hamiltonian in the 2D phase space).

Using the total energy integral Eq. (22) to compute $\partial u_{x} / \partial u_{j}^{ \pm}(j=e, p)$, and using the transverse momentum Eqs. (6) we obtain the longitudinal structure equation (see paper I):

$$
\begin{aligned}
\frac{d u_{x}}{d x}= & -\frac{u_{x}}{(\mu+1)\left(u_{x}^{2}-c_{s}^{2}\right)} \\
& \left(\frac{M_{A e}^{2} u_{x 0}(1+\mu)}{\cos \theta} u_{e} u_{p} \sin \phi+\operatorname{Im}\left[\delta_{0}\left(u_{e}^{-}-u_{p}^{-}\right)\right]\right),
\end{aligned}
$$

where

$c_{s}^{2}=\frac{u_{x 0}^{2}}{\mu+1}\left(\frac{\hat{u}_{x}^{1-\gamma_{e}}}{M_{e 0}^{2}}+\frac{\mu \hat{u}_{x}^{1-\gamma_{p}}}{M_{p 0}^{2}}\right) \equiv \frac{\gamma_{e} p_{e}+\gamma_{p} p_{p}}{\left(m_{e}+m_{p}\right) n U^{2}}$.

Note that $c_{s}$ is a normalized version of the sound speed for the combined electron-proton fluid. When the numerator and denominator in Eq. (31) are simultaneously zero defines the sonic critical point(s) of the flow. The locus $u_{x}=c_{s}$ in general defines values of $u_{x}$ where $d u_{x} / d x \rightarrow \infty$ and is related to shock formation phenomena.

Below we describe the variational and Hamiltonian formulations of the transverse electron and proton momentum Eqs. (6) obtained in paper I, which are used in the analysis of the integrable solutions $\left(\delta_{0}=0\right)$ in Sects. 4 and 5.

\section{Proposition 3.1}

The transverse electron and proton momentum Eqs. (6) can be obtained by extremizing the action:

$A=\int_{-\infty}^{\infty} \mathcal{L} d x$

with respect to $u_{e}^{ \pm}$and $u_{p}^{ \pm}$, where the Lagrangian density $\mathcal{L}$ is given by:

$\mathcal{L}=u_{e}^{-} \frac{d u_{e}^{+}}{d x}-\mu^{2} u_{p}^{-} \frac{d u_{p}^{+}}{d x}+\frac{2 i(\mu+1) \cos \theta}{u_{x 0}}\left(P_{x}^{(w)}-P_{x 0}^{(w)}\right)$,

subject to the constraint that the energy integral Eq. (22): $\varepsilon=\varepsilon_{0}$ is satisfied. In other words, $u_{x}=u_{x}\left(u_{j}^{ \pm}\right)$is an implicit function of the $u_{j}^{ \pm}(j=e, p)$ given by solving the energy integral Eq. (22) for $u_{x}$ in terms of the $u_{j}^{ \pm}$.
Comment: The Lagrangian $\mathcal{L}$ in Eq. (34) is equivalent to the Lagrangian $\mathcal{L}^{\prime}$ (meaning it has the same Euler-Lagrange equations) given by:

$$
\begin{aligned}
\mathcal{L}^{\prime}= & i\left(\mu^{2} \mathbf{u}_{p} \cdot \nabla \times \mathbf{u}_{p}-\mathbf{u}_{e} \cdot \nabla \times \mathbf{u}_{e}\right. \\
& \left.+\frac{2(\mu+1) \cos \theta}{u_{x 0}}\left(P_{x}^{(w)}-P_{x 0}^{(w)}\right)\right) .
\end{aligned}
$$

The Lagrangians $\mathcal{L}$ and $\mathcal{L}^{\prime}$ differ by a perfect derivative. The Lagrangian Eq. (35) consists of three terms representing contributions from the proton fluid helicity $\mu^{2} \mathbf{u}_{p} \cdot \nabla \times \mathbf{u}_{p}$, the electron helicity $-\mathbf{u}_{e} \cdot \nabla \times \mathbf{u}_{e}$, and the longitudinal momentum flux integral $P_{x}^{(w)}$. It is assumed in Eq. (33)-Eq. (35) that the energy integral $\varepsilon=\varepsilon_{0}$ acts a constraint on the dynamics. One can also write down a Lagrangian $\mathcal{L}^{\prime \prime}$ equivalent to Eq. (35), by using the generalized helicities of each species (see paper I, Appendix C).

\section{Proposition 3.2}

The transverse electron and proton momentum Eqs. (6) can be expressed in the Hamiltonian form:

$\frac{d q_{j}}{d x}=\frac{\partial \mathcal{H}}{\partial p_{j}}, \quad \frac{d p_{j}}{d x}=-\frac{\partial \mathcal{H}}{\partial q_{j}}, \quad j=1,2$,

where

$\left(q_{1}, p_{1}\right)=\left(u_{e}^{+}, u_{e}^{-}\right), \quad\left(q_{2}, p_{2}\right)=\left(u_{p}^{+},-\mu^{2} u_{p}^{-}\right)$,

are the canonical coordinates and

$\mathcal{H}=-\frac{2 i(\mu+1) \cos \theta}{u_{x 0}}\left(P_{x}^{(w)}-P_{x 0}^{(w)}\right)$.

Thus, the Hamiltonian Eq. (38) is equivalent, modulo a trivial scaling constant of $-2 i(\mu+1) \cos \theta / u_{x 0}$ to the total, longitudinal momentum flux $P_{x}^{(w)}$ (note that the momentum flux constant $P_{x 0}^{(w)}$ is not essential). Here the $x$-component of the fluid velocity $u_{x}$ is given implicitly by solving the energy integral Eq. (22) for $u_{x}=u_{x}\left(u_{j}^{ \pm}\right)$in terms of the $u_{j}^{ \pm}(j=e, p)$. The result Eq. (38) is reminiscent of the work of Bridges (1992), who showed that the spatial Hamiltonian for nonlinear travelling water waves is the $x$-momentum flux integral.

\section{Poisson brackets and integrable dynamics}

In this section, we discuss the role of Poisson brackets in reducing the Hamiltonian system Eq. (36)-Eq. (38) governing the transverse electron and proton dynamics (i.e. Eqs. 6) in the non-integrable and integrable cases. In the integrable cases (i.e. $\delta_{0}=0$ ), there is a reduction of the phase space possible from a four dimensional phase space to a two dimensional phase space, which is associated with the two Lie symmetries $X_{1}$ ( $x$-translation symmetry) and $X_{2}$ (rotational symmetry) (see paper I). The fourth order Hamiltonian system Eq. (6) for $\delta_{0}=0$ has two Lie symmetries and hence 
is completely integrable by Darboux's theorem (e.g. Olver, 1993). The condition $\delta_{0}=0$ in fact forces the dynamics to be restricted to a single Hamiltonian contour in the $(\phi, w)$ phase plane, where $w=u_{e}^{2}$ and $\phi=\phi_{p}-\phi_{e}$.

Using the amplitude and phase form of the complex transverse velocities $u_{j}^{ \pm}=u_{j} \exp \left( \pm i \phi_{j}\right)(j=e, p)$, the Hamiltonian density Eq. (38) may be written in the form:

$\mathcal{H}=-\frac{2 i(\mu+1) \cos \theta}{u_{x 0}}\left(P\left(\hat{u}_{x}\right)+P_{B}-P_{x 0}^{(w)}\right)$,

where

$$
\begin{aligned}
P_{B}= & \frac{M_{\mathrm{Ae} 0}^{2}}{2(\mu+1) \cos ^{2} \theta}\left(u_{e}^{2}+\mu^{2} u_{p}^{2}+2 \mu u_{p} u_{e} \cos \phi\right) \\
& +\frac{u_{x 0}}{(\mu+1) \cos \theta} \operatorname{Re}\left[\delta_{0}^{*}\left(u_{e}^{+}+\mu u_{p}^{+}\right)\right] \\
& +\frac{u_{x 0}^{2}\left|\delta_{0}\right|^{2}}{2(\mu+1) M_{\mathrm{Ae} 0}^{2}},
\end{aligned}
$$

and $P\left(\hat{u}_{x}\right)$ is the fluid dynamical momentum flux given by Eq. (18). In the general case, $\delta_{0} \neq 0$, the Hamiltonian density Eq. (39) can be written in terms of the four variables $\left(u_{e}, u_{p}, \phi, \tilde{\phi}\right)$ where $\phi=\phi_{p}-\phi_{e}$ and $\tilde{\phi}=\phi_{p}+\phi_{e}$ [note that $\phi_{p}=(\phi+\tilde{\phi}) / 2$ and $\left.\phi_{e}=(\tilde{\phi}-\phi) / 2\right]$. The $x$-component of the fluid velocity $u_{x}=u_{x}\left(u_{e}, u_{p}\right)$ can be determined by solving the energy integral Eq. (22) for $u_{x}$ in terms of $u_{e}$ and $u_{p}$.

In the integrable case $\delta_{0}=0$, the Hamiltonian density Eq. (39) depends only on $\left(u_{e}, u_{p}, \phi\right)$. However, by using the rotational integral $\mathcal{R}=u_{e}^{2}-\mu^{2} u_{p}^{2}, \mathcal{H}$ may be written in the form $\mathcal{H}=\hat{\mathcal{H}}(\phi, w)$ where $\phi=\phi_{p}-\phi_{e}$ and $w=u_{e}^{2}$ are the canonical variables in a reduced phase space.

In Sect. 4.1, we show how the phase space shrinks from a four dimensional phase space to a two dimensional phase space in the integrable cases, by using appropriate transformations of the phase space variables describing the Poisson bracket. Section 4.2 discusses the integration of the equations in the integrable cases when $\delta_{0}=0$.

\subsection{Poisson brackets and variable transformations}

Hamilton's Eqs. (36-37) can be written in the Poisson bracket form:

$\frac{d q_{k}}{d x}=\left\{q_{k}, H\right\}, \quad \frac{d p_{k}}{d x}=\left\{p_{k}, H\right\}$.

Here

$H=\int_{-\infty}^{\infty} d x \mathcal{H}$

is the Hamiltonian functional and

$\{F, G\}=\int_{-\infty}^{\infty} \sum_{k=1}^{2}\left(\frac{\delta F}{\delta q_{k}} \frac{\delta G}{\delta p_{k}}-\frac{\delta F}{\delta p_{k}} \frac{\delta G}{\delta q_{k}}\right) d x$, is the Poisson bracket for functionals. In this formulation

$$
\begin{aligned}
& p_{j}(x)=\int_{-\infty}^{\infty} p_{j}\left(x^{\prime}\right) \delta\left(x^{\prime}-x\right) d x^{\prime}, \\
& q_{j}(x)=\int_{-\infty}^{\infty} q_{j}\left(x^{\prime}\right) \delta\left(x^{\prime}-x\right) d x^{\prime}
\end{aligned}
$$

define the functionals $p_{j}(x)$ and $q_{j}(x)$. From Eq. (44)

$$
\frac{\delta p_{j}(x)}{\delta p_{k}\left(x^{\prime}\right)}=\delta_{j k} \delta\left(x^{\prime}-x\right), \quad \frac{\delta q_{j}(x)}{\delta q_{k}\left(x^{\prime}\right)}=\delta_{j k} \delta\left(x^{\prime}-x\right),
$$

where $\delta_{j k}$ is the Kronecker-delta symbol and $\delta\left(x^{\prime}-x\right)$ is the Dirac-delta distribution.

Using the definition Eq. (43) for the Poisson bracket in Eq. (41) we obtain:

$\frac{d q_{k}}{d x}=\frac{\delta H}{\delta p_{k}} \quad$ and $\quad \frac{d p_{k}}{d x}=-\frac{\delta H}{\delta q_{k}}$.

In the present example, $\delta H / \delta p_{k}=\partial \mathcal{H} / \partial p_{k}$ and $\delta H / \delta q_{k}=\partial \mathcal{H} / \partial q_{k}$, and hence Eq. (46) are equivalent to the classical Hamiltonian Eq. (36).

The Poisson bracket Eq. (43), written in terms of the canonical variables Eq. (37) reduces to:

$$
\begin{aligned}
\{F, G\}= & \int_{-\infty}^{\infty}\left[\left(\frac{\delta F}{\delta u_{e}^{+}} \frac{\delta G}{\delta u_{e}^{-}}-\frac{\delta F}{\delta u_{e}^{-}} \frac{\delta G}{\delta u_{e}^{+}}\right)\right. \\
& \left.-\frac{1}{\mu^{2}}\left(\frac{\delta F}{\delta u_{p}^{+}} \frac{\delta G}{\delta u_{p}^{-}}-\frac{\delta F}{\delta u_{p}^{-}} \frac{\delta G}{\delta u_{p}^{+}}\right)\right] d x .
\end{aligned}
$$

The Poisson bracket Eq. (47) can be transformed into a variety of different forms, by changing the physical variables used in the Poisson bracket. This is useful in describing the reduced Hamiltonian dynamics associated with the integrable cases where $\delta_{0}=0$, where a reduction in the number of phase space variables can be effected. To this end, we use the amplitude and phase form of the transverse fluid velocities $u_{j}^{ \pm}=u_{j} \exp \left( \pm i \phi_{j}\right),(j=e, p)$. Using the variational derivative transformations:

$\frac{\delta F}{\delta u_{j}^{ \pm}}=\frac{1}{2} \exp \left(\mp i \phi_{j}\right)\left(\frac{\delta F}{\delta u_{j}} \mp \frac{i}{u_{j}} \frac{\delta F}{\delta \phi_{j}}\right)$,

the Poisson bracket Eq. (47) becomes:

$$
\begin{aligned}
\{F, G\}= & \frac{1}{2 i} \int\left[\frac{1}{u_{e}}\left(\frac{\delta F}{\delta \phi_{e}} \frac{\delta G}{\delta u_{e}}-\frac{\delta F}{\delta u_{e}} \frac{\delta G}{\delta \phi_{e}}\right)\right. \\
& \left.-\frac{1}{\mu^{2} u_{p}}\left(\frac{\delta F}{\delta \phi_{p}} \frac{\delta G}{\delta u_{p}}-\frac{\delta F}{\delta u_{p}} \frac{\delta G}{\delta \phi_{p}}\right)\right] d x .
\end{aligned}
$$

Similarly, using the variables:

$\tilde{\phi}=\phi_{p}+\phi_{e}, \quad \phi=\phi_{p}-\phi_{e}, \quad \chi_{j}=u_{j}^{2}, \quad j=e, p$, 
and the variational derivative transformations

$$
\begin{aligned}
& \frac{\delta F}{\delta u_{j}}=2 u_{j} \frac{\delta F}{\delta \chi_{j}}, \quad \frac{\delta F}{\delta \phi_{p}}=\frac{\delta F}{\delta \tilde{\phi}}+\frac{\delta F}{\delta \phi}, \\
& \frac{\delta F}{\delta \phi_{e}}=\frac{\delta F}{\delta \tilde{\phi}}-\frac{\delta F}{\delta \phi}, \quad j=e, p,
\end{aligned}
$$

the bracket Eq. (49) transforms to the form:

$$
\begin{aligned}
\{F, G\}= & i \int_{-\infty}^{\infty}\left\{\frac{\delta F}{\delta \phi}\left(\frac{\delta G}{\delta \chi_{e}}+\frac{1}{\mu^{2}} \frac{\delta G}{\delta \chi_{p}}\right)\right. \\
& -\frac{\delta G}{\delta \phi}\left(\frac{\delta F}{\delta \chi_{e}}+\frac{1}{\mu^{2}} \frac{\delta F}{\delta \chi_{p}}\right) \\
& -\left[\frac{\delta F}{\delta \tilde{\phi}}\left(\frac{\delta G}{\delta \chi_{e}}-\frac{1}{\mu^{2}} \frac{\delta G}{\delta \chi_{p}}\right)\right. \\
& \left.\left.-\frac{\delta G}{\delta \tilde{\phi}}\left(\frac{\delta F}{\delta \chi_{e}}-\frac{1}{\mu^{2}} \frac{\delta F}{\delta \chi_{p}}\right)\right]\right\} d x .
\end{aligned}
$$

Introducing the variables:

$$
\chi_{ \pm}=\chi_{e} \pm \mu^{2} \chi_{p}
$$

to replace $\chi_{e}$ and $\chi_{p}$ (note that $\chi_{-}=u_{e}^{2}-\mu^{2} u_{p}^{2} \equiv \mathcal{R}=$ const. is the rotational integral of Eq. (6) if $\delta_{0}=0$ ), and using the transformations:

$$
\frac{\delta F}{\delta \chi_{e}}=\frac{\delta F}{\delta \chi_{+}}+\frac{\delta F}{\delta \chi_{-}}, \quad \frac{\delta F}{\delta \chi_{p}}=\mu^{2}\left(\frac{\delta F}{\delta \chi_{+}}-\frac{\delta F}{\delta \chi_{-}}\right),
$$

the bracket Eq. (52) reduces to the canonical form:

$$
\begin{aligned}
\{F, G\}= & 2 i \int_{-\infty}^{\infty}\left(\frac{\delta F}{\delta \chi_{-}} \frac{\delta G}{\delta \tilde{\phi}}-\frac{\delta F}{\delta \tilde{\phi}} \frac{\delta G}{\delta \chi_{-}}\right. \\
& \left.+\frac{\delta F}{\delta \phi} \frac{\delta G}{\delta \chi_{+}}-\frac{\delta F}{\delta \chi_{+}} \frac{\delta G}{\delta \phi}\right) d x
\end{aligned}
$$

which shows that $\left(\chi_{-}, \tilde{\phi}\right)$ and $\left(\phi, \chi_{+}\right)$are canonically conjugate coordinates.

Using the Poisson bracket Eq. (55) we obtain the alternative Hamiltonian formulation of Eq. (36):

$\frac{d \phi}{d x}=\frac{\partial H_{2}}{\partial \chi_{+}}, \quad \frac{d \chi_{+}}{d x}=-\frac{\partial H_{2}}{\partial \phi}$,
$\frac{d \chi_{-}}{d x}=\frac{\partial H_{2}}{\partial \tilde{\phi}}, \quad \frac{d \tilde{\phi}}{d x}=-\frac{\partial H_{2}}{\partial \chi_{-}}$,

where

$H_{2}=\frac{4(\mu+1) \cos \theta}{u_{x 0}} P_{x}^{(w)}$,

is the Hamiltonian (i.e. $H_{2}=2 i \mathcal{H}$ where we neglect $P_{x 0}^{(w)}$ in Eq. 38).

In the integrable case, $\chi_{-}=u_{e}^{2}-\mu^{2} u_{p}^{2}=$ const., $\delta \chi_{-}=0$, and the bracket Eq. (55) reduces to the simplified form:

$\{F, G\}=2 i \int_{-\infty}^{\infty}\left(\frac{\delta F}{\delta \phi} \frac{\delta G}{\delta \chi_{+}}-\frac{\delta F}{\delta \chi_{+}} \frac{\delta G}{\delta \phi}\right) d x$.
The result Eq. (58) is an example of Hamiltonian reduction (e.g. Marsden and Ratiu, 1994). It shows that the four dimensional phase space spanned by $\left(\chi_{-}, \tilde{\phi}\right)$ and $\left(\phi, \chi_{+}\right)$in the integrable case reduces to a two dimensional submanifold governed by the variables $\phi$ and $\chi_{+}$, on which both $\mathcal{H}$ and $\chi_{-}$are constant. To make it more explicit, that the manifold is two dimensional, we note that

$$
\frac{\delta G}{\delta \chi_{+}}=\frac{1}{2}\left(\frac{\delta G}{\delta \chi_{e}}+\frac{1}{\mu^{2}} \frac{\delta G}{\delta \chi_{p}}\right)=\frac{1}{2} \frac{\delta \hat{G}}{\delta \chi_{e}},
$$

where $\hat{G}$ is the functional obtained by setting $\chi_{p}=\left(\chi_{e}-\right.$ $\left.\chi_{-}\right) / \mu^{2}$ in $G\left(\chi_{e}, \chi_{p}, \phi, \tilde{\phi}\right)$ where $\chi_{-}=$const. (i.e. $\hat{G}$ is a functional of $\chi_{e}$ and $\phi$, and $\tilde{\phi}$ does not play a role in the Hamiltonian dynamics). Using Eq. (59), the Poisson bracket Eq. (58) reduces to:

$\{F, G\}=i \int_{-\infty}^{\infty}\left(\frac{\delta \hat{F}}{\delta \phi} \frac{\delta \hat{G}}{\delta \chi_{e}}-\frac{\delta \hat{F}}{\delta \chi_{e}} \frac{\delta \hat{G}}{\delta \phi}\right) d x$.

The bracket Eq. (60) describes the integrable dynamics on the $\left(\phi, \chi_{e}\right)$ phase space, where the functionals $\hat{F}$ and $\hat{G}$ depend on $\phi$ and $\chi_{e}$.

\subsection{Integrable Cases: $\delta_{0}=0$}

In general, for a spatial Hamiltonian system, the evolution of a physical variable $\psi$ is given by the Poisson bracket equation $\psi_{x}=\{\psi, H\}$. Thus, $w_{x}=\{w, H\}$ and $\phi_{x}=\{\phi, H\}$ where $w=u_{e}^{2} \equiv \chi_{e}$. Using the Poisson bracket Eq. (60) we obtain Hamilton's equations:

$$
\begin{aligned}
\frac{d w}{d x}=-\frac{\partial H_{0}}{\partial \phi}= & 2 \frac{M_{\mathrm{Ae} 0}^{2}}{u_{x 0} \cos \theta} \mu u_{p} u_{e} \sin \phi \\
\frac{d \phi}{d x}=\frac{\partial H_{0}}{\partial w}= & \frac{M_{\mathrm{Ae} 0}^{2}}{u_{x 0} \cos \theta}\left[2+\left(\frac{\mu u_{p}}{u_{e}}+\frac{u_{e}}{\mu u_{p}}\right) \cos \phi\right] \\
& -\frac{(\mu+1) \cos \theta}{\mu u_{x}}
\end{aligned}
$$

for $w$ and $\phi$, where $H_{0}=i \mathcal{H}$ and $\mathcal{H}$ is the Hamiltonian density for $\delta_{0}=0$. If we omit the non-essential integration constant $P_{x 0}^{(w)}$ then

$$
\begin{aligned}
H_{0}= & \frac{M_{\mathrm{Ae} 0}^{2}}{u_{x 0} \cos \theta}\left(u_{e}^{2}+\mu^{2} u_{p}^{2}+2 \mu u_{p} u_{e} \cos \phi\right) \\
& +\frac{2(\mu+1) \cos \theta}{u_{x 0}} P\left(\hat{u}_{x}\right) .
\end{aligned}
$$

Consider the longitudinal velocity structure Eq. (31):

$\frac{d u_{x}}{d x}=-\frac{M_{\mathrm{Ae} 0}^{2} u_{x} u_{e} u_{p} \sin \phi}{u_{x 0} \cos \theta\left(u_{x}^{2}-c_{s}^{2}\right)}$.

We show below that the righthand side of Eq. (64) can be expressed solely as a function of $u_{x}$, and hence can be integrated to give $x$ as a function of $u_{x}$. The solution for $u_{x}$ 
as a function of $x$ is not necessarily a 1-1 function. For example, $u_{x}$ may become a double valued function of $x$ if the solution passes through the sonic point where $u_{x}=c_{s}$. In this case a shock must be inserted into the flow in order to obtain a single valued weak solution.

\section{Proposition 4.1}

The longitudinal structure Eq. (64) may be reduced to the separable form:

$\frac{d u_{x}}{d x}=-\frac{\sigma(\mu+1) u_{x 0} \cos \theta u_{x} \sqrt{R\left(u_{x}\right)}}{\mu\left(u_{x}^{2}-c_{s}^{2}\right)}$.

where $\sigma=\operatorname{sgn}(\tan (\phi / 2)$. In particular, the integral of Eq. (65) is of the form:

$x=X\left(u_{x}\right)=-\int^{u_{x}} \frac{\mu\left(u_{x}^{2}-c_{s}^{2}\right) d u_{x}}{\sigma(\mu+1) u_{x 0} \cos \theta u_{x} \sqrt{R\left(u_{x}\right)}}+$ const.

where it is assumed that the integral in Eq. (66) is integrable.

The function $R\left(u_{x}\right)$ is defined by the equations:

$$
\begin{aligned}
& R\left(u_{x}\right)=N\left(u_{x}\right) D\left(u_{x}\right), \\
& N\left(u_{x}\right)=\frac{M_{\mathrm{Ae} 0}^{2}\left(u_{e}+\mu u_{p}\right)^{2}}{2(\mu+1) \cos ^{2} \theta}-\left[H-P\left(\hat{u}_{x}\right)\right], \\
& D\left(u_{x}\right)=H-P\left(\hat{u}_{x}\right)-\frac{M_{\mathrm{Ae} 0}^{2}\left(u_{e}-\mu u_{p}\right)^{2}}{2(\mu+1) \cos ^{2} \theta}, \\
& \tau^{2}=\tan ^{2}\left(\frac{\phi}{2}\right)=\frac{N\left(u_{x}\right)}{D\left(u_{x}\right)}, \quad \sigma=\operatorname{sgn}(\tau), \\
& \hat{u}_{e}^{2}=\mu\left(\frac{\hat{\mathcal{R}}+2 \mu \hat{\varepsilon}_{H T}}{\mu(\mu+1)}-\hat{u}_{x}^{2}-\frac{2 W\left(\hat{u}_{x}\right)}{\mu+1}\right), \\
& \hat{u}_{p}^{2}=\frac{1}{\mu}\left(\frac{2 \hat{\varepsilon}_{H T}-\hat{\mathcal{R}}}{\mu+1}-\hat{u}_{x}^{2}-\frac{2 W\left(\hat{u}_{x}\right)}{\mu+1}\right) .
\end{aligned}
$$

where

$$
\begin{aligned}
\hat{\mathcal{R}} & \equiv \frac{\mathcal{R}}{u_{x 0}^{2}}=\hat{u}_{e}^{2}-\mu^{2} \hat{u}_{p}^{2}, \\
\hat{\varepsilon}_{H T} & \equiv \frac{\varepsilon_{H T}}{u_{x 0}^{2}}=\frac{\varepsilon_{0}}{u_{x_{0}}^{2}}+\frac{1}{2}(\mu+1) \hat{V}_{H T}^{2},
\end{aligned}
$$

are the normalized rotational integral Eq. (30) and the energy integral in the dHT frame respectively. The explicit form for $R\left(u_{x}\right)$ from Eqs. (67-72) is:

$$
\begin{aligned}
R\left(u_{x}\right)= & \frac{2 \mu M_{\mathrm{Ae} 0}^{2} u_{x 0}^{2}}{(\mu+1) \cos ^{2} \theta}\left[H-P\left(\hat{u}_{x}\right)\right] \\
& {\left[\frac{4 \mu \hat{\varepsilon}_{H T}+(1-\mu) \hat{\mathcal{R}}}{2 \mu(\mu+1)}-\hat{u}_{x}^{2}-\frac{2 W\left(\hat{u}_{x}\right)}{\mu+1}\right] } \\
& -\left[H-P\left(\hat{u}_{x}\right)\right]^{2}-\frac{M_{\mathrm{Ae} 0}^{4} u_{x 0}^{4} \hat{\mathcal{R}}^{2}}{4(\mu+1)^{2} \cos ^{4} \theta},
\end{aligned}
$$

where

$$
\begin{aligned}
H=\frac{H_{0} u_{x 0}}{2(\mu+1) \cos \theta} \equiv & P\left(\hat{u}_{x}\right)+\frac{M_{\mathrm{Ae} 0}^{2}}{2(\mu+1) \cos ^{2} \theta} \\
& \left(u_{e}^{2}+\mu^{2} u_{p}^{2}+2 \mu u_{p} u_{e} \cos \phi\right),
\end{aligned}
$$

is a renormalised version of the Hamiltonian integral $H_{0}$ and

$W\left(\hat{u}_{x}\right)=\frac{\hat{u}_{x}^{1-\gamma_{e}}}{\left(\gamma_{e}-1\right) M_{e 0}^{2}}+\frac{\mu \hat{u}_{x}^{1-\gamma_{p}}}{\left(\gamma_{p}-1\right) M_{p 0}^{2}}$,

is the enthalpy contribution to the energy integral Eq. (22), $P\left(\hat{u}_{x}\right)$ is the longitudinal, fluid dynamical $x$-momentum flux of the proton and electron fluids Eq. (18), and $c_{s}^{2}$ is the square of the combined sound speed Eq. (32) which is a function of $u_{x}$.

The value of $\cos \phi$ throughout the wave from Eq. (75) is given by

$\cos \phi=\frac{2(\mu+1) \cos ^{2} \theta M_{\mathrm{Ae} 0}^{-2}\left[H-P\left(\hat{u}_{x}\right)\right]-u_{e}^{2}-\mu^{2} u_{p}^{2}}{2 \mu u_{p} u_{e}}$,

which can be written solely as a function of $u_{x}$.

Proof: The main idea behind the proof is to express $u_{e}, u_{p}$ and $\sin \phi$ in the $x$-structure Eq. (64) in terms of $u_{x}$. To derive Eq. (71) and (72) for $u_{e}\left(u_{x}\right)$ and $u_{p}\left(u_{x}\right)$ we note that the energy integral Eq. (22) may be written in the form:

$\hat{\varepsilon}_{H T}=\frac{1}{2}(\mu+1) \hat{u}_{x}^{2}+W\left(\hat{u}_{x}\right)+\frac{1}{2}\left(\hat{u}_{e}^{2}+\mu \hat{u}_{p}^{2}\right)$,

Simultaneously solving the first equation in Eq. (73) and Eq. (78) for $\hat{u}_{e}^{2}$ and $\hat{u}_{p}^{2}$ gives Eq. (71) and Eq. (72).

To derive Eq. (70) for $\tau=\tan (\phi / 2)$, substitute the half angle trignometric formula $\cos \phi=\left(1-\tau^{2}\right) /\left(1+\tau^{2}\right)$ for $\cos \phi$ in the Hamiltonian integral Eq. (75), and solve for $\tau^{2}$ in terms of $H$ and the other variables, to obtain $\tau^{2}=N\left(u_{x}\right) / D\left(u_{x}\right)$ where $N$ and $D$ are given by Eq. (68) and Eq. (69). A straightforward calculation gives:

$\sin \phi=\frac{2 \tau}{1+\tau^{2}}=\frac{\sigma(\mu+1) \cos ^{2} \theta \sqrt{N\left(u_{x}\right) D\left(u_{x}\right)}}{M_{\mathrm{Ae} 0}^{2} \mu u_{p} u_{e}}$.

Using the identity Eq. (79) in the structure Eq. (64) gives the differential Eq. (65) in which $d u_{x} / d x$ is a function of $u_{x}$.

In the integrable case, the differential equation for $\tilde{\phi}$ Eq. (28) reduces to:

$$
\frac{d \tilde{\phi}}{d x}=\frac{M_{\mathrm{Ae} 0}^{2} u_{x 0}}{\cos \theta}\left(\frac{u_{e}}{\mu u_{p}}-\frac{\mu u_{p}}{u_{e}}\right) \cos \phi+\frac{(\mu-1) \cos \theta}{\mu u_{x}} .
$$


The right-hand side of Eq. (80) can be written solely in terms of $u_{x}$. Thus, $d \tilde{\phi} / d u_{x}=d \tilde{\phi} / d x /\left(d u_{x} / d x\right)=F\left(u_{x}\right)$ is also a function $F\left(u_{x}\right)$ of $u_{x}$, and hence $\tilde{\phi}=\int F\left(u_{x}\right) d u_{x}$ is also a function of $u_{x}$. The net upshot of the above analysis is that $x=X\left(u_{x}\right), \phi=\phi\left(u_{x}\right), u_{e}=u_{e}\left(u_{x}\right), u_{p}=u_{p}\left(u_{x}\right)$ and $\tilde{\phi}=\tilde{\phi}\left(u_{x}\right)$, and hence the system Eq. (6) for the integrable case can be expressed in terms of integrals of functions of $u_{x}$ and in terms of ordinary functions of $u_{x}$. Note that the proton and electron phases $\phi_{p}=(\tilde{\phi}+\phi) / 2$ and $\phi_{e}=(\tilde{\phi}-\phi) / 2$ can be expressed in terms of $\phi$ and $\tilde{\phi}$. One can easily show that $N$ and $D$ are non-negative. This completes the proof.

\section{Solution examples}

In this section we give numerical solution examples of oblique travelling waves for the integrable cases for which $\delta_{0}=0$. We concentrate on the cold plasma solutions. We give a discussion of the nature of the critical points for the hot plasma case as well. For the integrable cases, the integrability condition $\delta_{0}=0$, (i.e. Eq. 14), is equivalent to setting the total complex, transverse momentum flux in the dHT frame, $P_{+}^{(d)}$, equal to zero. A detailed discussion of the integrability condition $\delta_{0}=0$ is given in Appendix A.

In general, the Hamiltonian $H$ in the cold plasma, integrable case $\delta_{0}=0$ has the form:

$H=u_{x} u_{x 0}+A\left(u_{e}^{2}+\mu^{2} u_{p}^{2}+2 \mu u_{p} u_{e} \cos \phi\right)$,

where

$$
\begin{aligned}
u_{e}^{2} & =u_{e 0}^{2}+\mu\left(u_{x 0}^{2}-u_{x}^{2}\right), \quad u_{p}^{2}=u_{p 0}^{2}+\frac{\left(u_{x 0}^{2}-u_{x}^{2}\right)}{\mu}, \\
A & =\frac{M_{\mathrm{Ae} 0}^{2} \sec ^{2} \theta}{2(\mu+1)} \equiv \frac{M_{A}^{2} u_{x 0}^{2}}{2(\mu+1)^{2}} .
\end{aligned}
$$

Alternatively, $H$ can be written in terms of the canonical variables $w=u_{e}^{2}$ and $\phi$ in the form:

$$
\begin{aligned}
H= & u_{x 0} u_{x}(w) \\
& +A\left(w+\mu^{2} u_{p}(w)^{2}+2 \mu w^{1 / 2} u_{p}(w) \cos \phi\right),
\end{aligned}
$$

where

$$
\begin{aligned}
\mu u_{p}(w) & =\left(\mu^{2} u_{p 0}^{2}-u_{e 0}^{2}+w\right)^{1 / 2}, \\
u_{x}(w) & =\left(u_{x 0}^{2}+\frac{\left(u_{e 0}^{2}-w\right)}{\mu}\right)^{1 / 2} .
\end{aligned}
$$

However, the integrability constraints Eq. (14) (i.e. $\delta_{0}=0$ ) require that the initial transverse electron and proton fluid velocities satisfy the Eqs.

$u_{e y 0}+\mu u_{p y 0}=0, \quad u_{e z 0}+\mu u_{p z 0}=b$, corresponding to zero, total transverse momentum fluxes for the system in the dHT frame. Using Eq. (86) (see Appendix A), it follows, that the Hamiltonian integral $H$ can only have one value, namely $H_{1}$, where

$H_{1}=u_{x 0}^{2}+\frac{\tan ^{2} \theta}{2 M_{A}^{2}}=u_{x 0}^{2}\left(1+\frac{\sin ^{2} \theta}{2(\mu+1) M_{\mathrm{Ae} 0}^{2}}\right)$.

Thus, the system trajectories in phase space satisfy the equation

$H(\phi(x), w(x))=H_{1}$,

which essentially fixes the solutions to lie on a particular invariant torus. Put another way, the solution trajectories in phase space consist of the locus of phase space points $(\phi, w)$ satisfying $H(\phi, w)=H_{1}$ (i.e. an $H_{1}$ contour level).

In the present paper, we restrict our attention to the class of integrable solutions with $\delta_{0}=0$ satisfying the initial conditions:

$$
\begin{aligned}
& \phi_{e 0}=\phi_{p 0}=\frac{\pi}{2}, \quad \phi_{0}=0, \quad \tilde{\phi}_{0}=\pi \\
& u_{e 0}+\mu u_{p 0}=\frac{(\mu+1) \tan \theta}{u_{x 0} M_{A}^{2}}=\frac{u_{x 0} \sin \theta \cos \theta}{M_{\mathrm{Ae} 0}^{2}}=b .
\end{aligned}
$$

A more general class of initial data with $\phi_{0} \neq 0$, satisfying the integrability condition $\delta_{0}=0$ is discussed in Appendix A.

In general, the class of solutions satisfying Eq. (87) and Eq. (89) includes solutions for which the transverse, complex velocities $u_{j 0}^{(w)+}=u_{j 0}^{(w) y}+i u_{j 0}^{(w) z}$ in the travelling wave frame are non-zero. However, in the special case of Eq. (89) for which

$$
\begin{aligned}
& u_{e 0}=u_{p 0}=\tan \theta, \quad M_{A}^{2} u_{x 0}=1, \\
& \phi_{e 0}=\phi_{p 0}=\frac{\pi}{2}, \quad \phi_{0}=0, \quad \tilde{\phi}_{0}=\pi .
\end{aligned}
$$

we obtain an integrable class of solutions satisfying $u_{j 0}^{(w)+}=0$ at $x=x_{0}$ in the wave frame. These solutions have similar boundary conditions to the oscilliton solutions investigated by Dubinin et al. (2003), for which $u_{j 0}^{(w)+}=0$ as $x_{0} \rightarrow-\infty$. For the initial data Eq. (90), the Hamiltonian integral Eq. can be written in the $(87)$ reduces to:

$$
H_{1}=u_{x 0}^{2}+\frac{1}{2} u_{x 0} \tan ^{2} \theta
$$

We first discuss the critical points of the differential equation system Eq. (61) and Eq. (62) for $w=u_{e}^{2}$ and $\phi$ governing the Hamiltonian dynamics, as well as the related Eqs. (64) and (80) for $d u_{x} / d x$ and $d \tilde{\phi} / d x$. We discuss the sonic points and critical points for the hot plasma case, followed by a more detailed discussion of the cold plasma case, which is the main focus of the present paper. This is followed by examples of the phase trajectories in $(\phi, w)$ phase space, obtained by plotting the contours of the Hamiltonian in the 
phase plane. We discuss in detail the longitudinal structure equation for $d u_{x} / d x$, and how the solutions depend on the roots of the cubic equation, $R\left(u_{x}\right)=0$ determining the values of $u_{x}$ for which $d u_{x} / d x=0$. Examples of the solutions for different initial data are presented.

\subsection{Critical points}

The basic differential equations governing the two-fluid travelling waves consist of the Hamiltonian Eqs. (61-62):

$$
\begin{aligned}
\frac{d w}{d x} & =-\frac{\partial H_{0}}{\partial \phi}=2 \frac{M_{\mathrm{Ae} 0}^{2}}{u_{x 0} \cos \theta} \mu u_{p} u_{e} \sin \phi \\
\frac{d \phi}{d x} & =\frac{\partial H_{0}}{\partial w} \\
& =\frac{M_{\mathrm{Ae} 0}^{2}}{u_{x 0} \cos \theta}\left[2+\left(\frac{\mu u_{p}}{u_{e}}+\frac{u_{e}}{\mu u_{p}}\right) \cos \phi\right] \\
& -\frac{(\mu+1) \cos \theta}{\mu u_{x}},
\end{aligned}
$$

where $w=u_{e}^{2}$,

$$
\begin{aligned}
& u_{e}^{2}=u_{e 0}^{2}+\mu\left(u_{x 0}^{2}-u_{x}^{2}-\frac{2 \Delta W}{\mu+1}\right), \\
& u_{p}^{2}=u_{p 0}^{2}+\frac{1}{\mu}\left(u_{x 0}^{2}-u_{x}^{2}-\frac{2 \Delta W}{\mu+1}\right), \\
& u_{e 0}^{2}=\mu^{2}\left[u_{p y 0}^{2}+\left(u_{p z 0}-\frac{\mu+1}{\mu} \frac{\tan \theta}{u_{x 0} M_{A}^{2}}\right)^{2}\right],
\end{aligned}
$$

and $W\left(u_{x}\right)$ is the combined enthalpy of the electron-proton plasma, given by Eq. (76), and $\Delta W=W\left(u_{x}\right)-W\left(u_{x 0}\right)$. Note that for the integrable case $\delta_{0}=0$, and $u_{e 0}^{2}$ can be expressed in terms of $u_{p y 0}$ and $u_{p z 0}$ as in Eq. (96).

For hot plasmas, with $\Delta W\left(u_{x}\right)$ a non-trivial function of $u_{x}, u_{e}^{2}$ and $u_{p}^{2}$ consist of a single hump-like function of $u_{x}$, that have maxima at the sonic point where $u_{x}^{2}=c_{s}^{2}$ and $c_{s}$ is the combined electron and proton sound speed given by Eq. (32). Thus, for a given value of $w=u_{e}^{2}$, there are in general two values of $u_{x}^{2}$, one with $u_{x}^{2}<c_{s}^{2}$ and one with $u_{x}^{2}>c_{s}^{2}$. Hence, there is a subsonic solution branch of the Hamiltonian, $H_{0}^{-}$with $u_{x}<c_{s}$ and a supersonic branch $H_{0}^{+}$, with $u_{x}>c_{s}$, in which the sonic line $u_{x}=c_{s}$ separates the two branches.

It is useful to supplement the Hamiltonian Eqs. (92-93) with equations for $d u_{x} / d x$ and $d \tilde{\phi} / d x$ (Eqs. (64 and 80)):

$$
\begin{aligned}
\frac{d u_{x}}{d x}= & -\frac{M_{\mathrm{Ae} 0}^{2} u_{x} u_{e} u_{p} \sin \phi}{u_{x 0} \cos \theta\left(u_{x}^{2}-c_{s}^{2}\right)}, \\
\frac{d \tilde{\phi}}{d x}= & \frac{M_{\mathrm{Ae} 0}^{2} u_{x 0}}{\cos \theta}\left(\frac{u_{e}^{2}-\mu^{2} u_{p}^{2}}{\mu u_{p} u_{e}}\right) \cos \phi \\
& +\frac{(\mu-1) \cos \theta}{\mu u_{x}} .
\end{aligned}
$$

Introducing the state vector (Ko et al. 2007, in preparation):

$$
\mathbf{W}=\left(x, u_{x}^{2}, \phi, \tilde{\phi}\right)^{t}
$$

Equations (92)-(93) and (97)-(98) can be written in the form:

$$
\frac{d \mathbf{W}}{d \lambda}=\left(\begin{array}{c}
u_{x}^{2}-c_{s}^{2} \\
-2 M_{\mathrm{Ae} 0}^{2} u_{x}^{2} u_{e} u_{p} \sin \phi /\left(u_{x 0} \cos \theta\right) \\
\left(u_{x}^{2}-c_{s}^{2}\right) d \phi / d x \\
\left(u_{x}^{2}-c_{s}^{2}\right) d \tilde{\phi} / d x
\end{array}\right) \equiv \mathbf{N},
$$

where $\mathbf{N}$ is the column vector on the right hand side of Eq. (100). In the first equation in Eq. (100), $d x / d \lambda=\left(u_{x}^{2}-c_{s}^{2}\right)$ defines a convenient parameter $\lambda$ along the solution curves, which enables one to pass through the sonic point, without encountering an infinite derivative during numerical integration. The critical points of the system Eq. (100) are points at which the components of the column vector $\mathbf{N}$ are simultaneously zero. This is the approach used by Ko et al. (2007) in a study of the integrable, travelling waves in the above model of hot electron and proton plasmas with $p_{e} \neq 0$ and $p_{p} \neq 0$.

In the present paper, we restrict our attention to cold electron-proton plasmas, in which the entropy $W\left(u_{x}\right)=W\left(u_{x 0}\right)=0$ and hence $\Delta W=0$ in Eqs. (94-95). In this case, there is a one-to-one relation between $u_{e}^{2}$ and $u_{x}^{2}$. In the cold plasma limit, the sound speeds $c_{j 0} \rightarrow 0$ and the sonic Mach numbers $M_{j 0} \rightarrow \infty(j=e, p)$, and the flow is supersonic throughout. The critical points in this case, are simply the points in $(\phi, w)$ phase space where

$\frac{d w}{d x}=-\frac{\partial H_{0}}{\partial \phi}=0 \quad$ and $\quad \frac{d \phi}{d x}=\frac{\partial H_{0}}{\partial w}=0$.

We note

(i). $d w / d x=0$ when $\phi=0, \pm \pi$ or $u_{e}=0$ or $u_{p}=0$,

(ii). $d \phi / d x=0$ when the right hand side of (93) is zero.

Consider the possibility of a critical point on the line $\phi=0$, for which $d w / d x=0$. For $\phi=0$,

$$
\frac{d \phi}{d x}=\frac{M_{A}^{2} u_{x 0} \cos \theta}{1+\mu} \frac{\left(u_{e}+\mu u_{p}\right)^{2}}{\mu u_{p} u_{e}}-\frac{(\mu+1) \cos \theta}{\mu u_{x}} \equiv f\left(u_{x}\right),
$$

where $f\left(u_{x}\right)$ is the function of $u_{x}$ obtained by using the solutions Eq. (94) and Eq. (95) for $u_{e}^{2}$ and $u_{p}^{2}$. Note that $u_{e} \geq 0$ and $u_{p} \geq 0$ are required for physical solutions for $u_{e}$ and $u_{p}$. From Eq. (94)

$u_{e}^{2}=0 \quad$ when $\quad u_{x}^{2}=u_{x 1}^{2}=u_{x 0}^{2}+u_{e 0}^{2} / \mu$.

Similarly from Eq. (95):

$u_{p}^{2}=0 \quad$ when $\quad u_{x}^{2}=u_{x 2}^{2}=u_{x 0}^{2}+\mu u_{p 0}^{2}$. 
From Eqs. (103-104):

$u_{x 1}^{2}-u_{x 2}^{2}=\frac{u_{e 0}^{2}-\mu^{2} u_{p 0}^{2}}{\mu} \equiv \frac{\mathcal{R}}{\mu}$,

where $\mathcal{R}$ is the rotational integral. In general $\mathcal{R}$ can be positive or negative. For the conditions Eq. (90) $\mathcal{R}=\left(1-\mu^{2}\right) \tan ^{2} \theta<0$ and $u_{x 2}>u_{x 1}$.

\section{Proposition 5.1}

There is a critical point on the line $\phi=0$ in the $\left(\phi, u_{x}\right)$ plane at $u_{x}=u_{x c}$ where $0<u_{x c}<u_{m}, u_{m}=\min \left(u_{x 1}, u_{x 2}\right)$, and $u_{x 1}$ and $u_{x 2}$ refer to the values of $u_{x}$ for which $u_{e}=0$ and $u_{p}=0$ respectively (note $u_{m}>u_{x 0}$ ). Furthermore, the critical point is a centre.

Proof: The proof follows by noting that

$$
\frac{d f}{d u_{x}}=\frac{M_{A}^{2} u_{x 0} \cos \theta u_{x} \mathcal{R}^{2}}{2(\mu+1) \mu^{2} u_{e}^{3} u_{p}^{3}}+\frac{(\mu+1) \cos \theta}{\mu u_{x}^{2}}>0,
$$

and by noting that $f\left(u_{x}\right) \rightarrow-\infty$ as $u_{x} \rightarrow 0$ and $f\left(u_{x}\right) \rightarrow \infty$ as $u \rightarrow u_{m}$. The proof that $\left(\phi, u_{x}\right)=\left(0, u_{x c}\right)$ is a centre critical point is given in Appendix B.

\section{Comment:}

The exact location of the centre critical point in Proposition 5.1 depends on the value of the parameters. For example, if $u_{e 0}=u_{p 0}=\tan \theta$ and $M_{A}^{2} u_{x 0}=1$ as in Eq. (90) then

$f\left(u_{x 0}\right)=\frac{(\mu+1) \cos \theta}{\mu u_{x 0}}\left(u_{x 0}-1\right)$.

Thus, if $u_{x 0}>1$ (i.e. for a sub-Alfvénic travelling wave with $\left.M_{A}<1\right)$ then $u_{x c}<u_{x 0}$. However, for a super-Alfvénic travelling wave with $M_{A}>1$ and $u_{x 0}<1, u_{x c}>u_{x 0}$. For an Alfvénic wave with $M_{A}=1$ and $u_{x 0}=1$, then $u_{x c}=1$.

\section{Proposition 5.2}

There are no critical points along the lines $\phi= \pm \pi$ in the $(\phi, w)$ phase plane.

Proof: For $\phi= \pm \pi, d w / d x=0$ in Eq. (92). Also from Eq. (93) on the lines $\phi= \pm \pi$ :

$$
\frac{d \phi}{d x}=-\left(\frac{M_{\mathrm{Ae} 0}^{2}\left(u_{e}-\mu u_{p}\right)^{2}}{u_{x 0} \cos \theta}+\frac{(\mu+1) \cos \theta}{\mu u_{x}}\right)<0 .
$$

Hence $d \phi / d x \neq 0$ along $\phi= \pm \pi$, and there are no critical points along $\phi= \pm \pi$. This completes the proof.

\subsubsection{Non-standard critical points and separatrices}

The critical point $\left(\phi_{1}, w_{1}\right)=\left(0, u_{e 1}^{2}\right)$ in Proposition 5.1 is a centre critical point. Inspection of Eqs. (92) and (93) for $d w / d x$ and $d \phi / d x$ reveals that points where $\phi \rightarrow \pm \pi / 2$ and either $u_{p} \rightarrow 0$ or $u_{e} \rightarrow 0$ may behave like critical points if approached from a specific direction in the $(\phi, w)$ phase space. These points are not standard critical points, since the behaviour of $d w / d x$ and $d \phi / d x$ near these points diverges if the points are approached from other directions. Below we discuss the behaviour of the solutions in the vicinity of the $\left(\phi_{2}, w_{2}\right)=(-\pi / 2,0)$ and at $\left(\phi_{3}, w_{3}\right)=(\pi / 2,0)$ in the $(\phi, w)$ plane. We restrict our attention to the critical points $(\phi, w)=( \pm \pi / 2,0)$ (it is straightforward to carry out a similar analysis for the points for which $\phi= \pm \pi / 2$ and $u_{p}=0$ ). It turns out that the Hamiltonian contours passing through these "critical points" act as separatrices in the $(\phi, w)$ phase space, separating those solutions which are bounded in $\phi$ from those which are not. Discussion of the conditions for separatrices to appear in the phase space, associated with these critical points are given in Appendix C. Note that as $u_{e} \rightarrow 0$, $d w / d x \rightarrow 0$ near these points. However, the behaviour of $d \phi / d x$ in Eq. (93) is strongly dependent on the direction of approach at these points, and depends on the limit of the ratio of $\mu u_{p} \cos \phi / u_{e}$ as both $\cos \phi \rightarrow 0$ and $u_{e} \rightarrow 0$ simultaneously. Using the perturbation expansion $\phi=-\pi / 2+\alpha_{2} u_{e}$ where $u_{e}<<1$, one finds that $d w / d x \rightarrow 0$ and and $d \phi / d x \rightarrow 0$ as one approaches $\left(\phi_{2}, w_{2}\right)$ along the ray

$$
\frac{\delta \phi}{\delta u_{e}}=\alpha_{2}=\frac{\left[(\mu+1)^{2}-2 \mu u_{x c 2}\left(M_{A}^{2} u_{x 0}\right)\right]}{\mu^{2} u_{x c 2} u_{p c 2}\left(M_{A}^{2} u_{x 0}\right)},
$$

where

$u_{x c 2}=\left(u_{x 0}^{2}+u_{e 0}^{2} / \mu\right)^{1 / 2}, \quad u_{p c 2}=\frac{\left(\mu^{2} u_{p 0}^{2}-u_{e 0}^{2}\right)^{1 / 2}}{\mu}$,

However, along the ray $\delta u_{e}=0, \delta \phi \neq 0$ and $d \phi / d x$ is unbounded. Hence in general, the solution trajectories skirt around $\left(\phi_{2}, w_{2}\right)$ except along the ray Eq. (109). Similarly, for $\left(\phi_{3}, w_{3}\right)=(\pi / 2,0), d \phi / d x \rightarrow 0$ and $d w / d x \rightarrow 0$ along the ray $\phi=\pi / 2-\alpha_{2} u_{e}$ (i.e. $\delta \phi / \delta u_{e}=-\alpha_{2}$ ). The above results Eqs. (109-110) apply to the case of a cold plasma (similar behaviour applies for a hot plasma), and it is assumed that the rotational integral $\mathcal{R}<0$.

\section{Proposition 5.3}

The separatrix passing through $(\phi, w)=( \pm \pi / 2,0)$ for cold plasma solutions, satisfying the integrability constraints Eq. (89) also passes through the points $(\phi, w)=(0,0)$ and $(\phi, w)=\left(0, u_{e 0}^{2}\right)$ where

$u_{e 0}=\frac{2(\mu+1) u_{x 0} \tan \theta}{(\mu+1)^{2} / \mu-\tan ^{2} \theta}$.

The proof is given in Appendix C. Note that $u_{e 0}+\mu u_{p 0}=b$, $u_{e 0}>0$ and $u_{p 0}>0$ must also be satisfied (see Appendix $\mathrm{C}$ for details).

\section{Comment:}

It is also possible to have a separatrix passing through the points $\phi= \pm \pi / 2$ and $u_{p}=0$. The conditions for this to apply are discussed in Appendix C.

\section{Proposition 5.4}

The condition for the centre critical point $\left(\phi_{1}, w_{1}\right)=\left(0, w_{1}\right)$ to be a stationary point of the differential equation system Eqs. (92-93) is that $d \phi / d x=0$ and 
$d w / d x=0$ simultaneously at the initial point $\left(\phi_{0}, u_{e 0}^{2}\right)$ where $\phi_{0}=0$ and $u_{x}=u_{x 0}$. This condition is satisfied if

$\tan ^{2} \theta=M_{A}^{2} u_{p 0} u_{e 0}$.

\section{Proposition 5.5}

The conditions Eq. (89) for an integrable solution and the condition Eq. (112) for $\left(\phi_{1}, w_{1}\right)=\left(0, w_{1}\right)$ to be a stationary critical point may be written in the form:

$$
\begin{aligned}
u_{e 0}+\mu u_{p 0} & =\frac{u_{x 0} \sin \theta \cos \theta}{M_{\mathrm{Ae} 0}^{2}}=b, \\
\mu u_{e 0} u_{p 0} & =\frac{\mu u_{x 0}^{2} \sin ^{2} \theta}{(\mu+1) M_{\mathrm{Ae} 0}^{2}} .
\end{aligned}
$$

Equations (113-114) are satisfied simultaneously if $u_{e 0}$ satisfies the quadratic equation:

$$
D \equiv u_{e 0}^{2}-\frac{u_{x 0} \sin \theta \cos \theta}{M_{\mathrm{Ae} 0}^{2}} u_{e 0}+\frac{\mu \sin ^{2} \theta}{(\mu+1) M_{\mathrm{Ae} 0}^{2}} u_{x 0}^{2}=0
$$

From Eqs. (113-114) we obtain:

$$
\begin{aligned}
u_{e 0}^{ \pm} & =\frac{u_{x 0} \sin \theta \cos \theta}{2 M_{\mathrm{Ae} 0}^{2}}\left(1 \pm \sqrt{\delta_{1}}\right), \\
\mu u_{p 0}^{ \pm} & =\frac{u_{x 0} \sin \theta \cos \theta}{2 M_{\mathrm{Ae} 0}^{2}}\left(1 \mp \sqrt{\delta_{1}}\right),
\end{aligned}
$$

as the solutions for $u_{e 0}$ and $u_{p 0}$, where

$\delta_{1}=1-\frac{4 \mu}{(\mu+1)} M_{\mathrm{Ae} 0}^{2} \sec ^{2} \theta$.

Comment: 1

The conditions for the stationary point for integrable solutions in Proposition 5.5 requires $\delta_{1}>0$. This condition requires that

$M_{\mathrm{Ae} 0} \sec \theta<\frac{1}{2} \sqrt{\frac{\mu+1}{\mu}}$.

\section{Comment: 2}

It turns out that the integrable solutions of the system of differential Eqs. (92-98) for the cold plasma case, can be classified in terms of whether $D>0, D<0$ or $D=0$, and on whether $\delta_{1}>0, \delta_{1}<0$ or $\delta_{1}=0$, where $D$ is given by Eq. (115) and $\delta_{1}$ by Eq. (117). The condition $D=0$ is in fact a condition for the function $R\left(u_{x}\right)$ in the longitudinal structure Eq. (65) for $d u_{x} / d x$ to have a double zero $u_{x}=u_{x 0}$ in the cold plasma case.

Comment: 3

It is instructive to write the function $D\left(u_{e 0}\right)$ in Eq. (115) in terms of the rotational integral $\mathcal{R}$. Taking into account the integrability constraint Eq. (89) for $\phi_{0}=0$, we obtain the two equations:

$u_{e 0}+\mu u_{p 0}=b, \quad \mathcal{R}=u_{e 0}^{2}-\mu^{2} u_{p 0}^{2}$.
From Eq. (120), we obtain the equations:

$u_{e 0}=\frac{\mathcal{R}+b^{2}}{2 b}, \quad \mu u_{p 0}=\frac{b^{2}-\mathcal{R}}{2 b}$,

for $u_{e 0}$ and $\mu u_{p 0}$. Because, we require $u_{e 0} \geq 0$ and $u_{p 0} \geq 0$ for a physical solution, then the rotational integral $\mathcal{R}$ in Eq. (121) must lie in the range:

$-b^{2} \leq \mathcal{R} \leq b^{2}$

The separatrix solutions associated with $u_{e 0}=0$ and $\mu u_{p 0}=0$ (Appendix C) correspond to $\mathcal{R}=-b^{2}$ and $\mathcal{R}=b^{2}$ respectively. Using Eq. (121) for $u_{e 0}$ in the expression Eq. (115) for $D\left(u_{e 0}\right)$ we obtain:

$D=\frac{\mathcal{R}^{2}-b^{4} \delta_{1}}{4 b^{2}}$

where $\delta_{1}$ is given by Eq. (118). The result Eq. (123) shows the important role played by the rotational integral $\mathcal{R}$ and $M_{\mathrm{Ae} 0} \sec \theta$ (i.e. $\left.\delta_{1}\right)$ in determining the roots of $R\left(u_{x}\right)$ in the longitudinal structure Eq. (65) for $d u_{x} / d x$ in the cold plasma limit.

\subsection{Hamiltonian contours}

In this section we give examples of the Hamiltonian trajectories in the $(\phi, w)$ phase space, for the integrable cold plasma solution cases satisfying one of the initial conditions Eq. (89) or Eq. (90). The integrability constraints Eq. (86) force the system trajectories to lie on a specific Hamiltonian contour, $H=H_{1}$, where $H_{1}$ depends on the initial data. We plot the system trajectories in phase space (the contours $H=H_{1}$ ), for a family of different Hamiltonian functions Eq. (84) obtained by varying a particular parameter in the Hamiltonian $H$ (e.g. $u_{x 0}$ ) whilst keeping the other parameters fixed.

Figure 1 shows a family of contours $H=H_{1}$ in the $(\phi, w)$ phase-plane, for a cold plasma satisfying the initial conditions Eq. (90), i.e., $M_{A}^{2} u_{x 0}=1, u_{e 0}=u_{p 0}=\tan \theta$, where $\theta=60^{\circ}$. For this initial data, the transverse velocities of the electrons and protons are zero at $x=x_{0}$ in the travelling wave frame, which is similar to the boundary conditions used by Dubinin et al. (2003). The figure shows the effect of varying the longitudinal flow speed $u_{x 0}$ from $u_{x 0}=0.0001$ in steps of 0.1 up to $u_{x 0}=2.0$. The $u_{x 0}=0.0001$ curve is the nearly horizontal curve passing through $w=3$. The parameter $u_{x 0}$ increases moving clockwise and downwards across the curves on the right hand side of the figure $\left(u_{x 0}=0.1(0.1) 0.4\right)$ until one encounters the separatrix $\left(u_{x 0} \approx 0.5\right)$, which is the contour with the cusps at $(\phi, w)=( \pm \pi / 2,0)$. For the separatrix,

$u_{x 0}=\frac{u_{e 0}\left[(\mu+1)^{2} / \mu-\tan ^{2} \theta\right]}{2(\mu+1) \tan \theta}$

(see Appendix C). Setting $u_{e}=\tan \theta=\sqrt{3}$ and $\mu=1836$ in Eq. (124) gives $u_{x 0}=0.4997 \approx 0.5$, for the value of $u_{x 0}$ for 


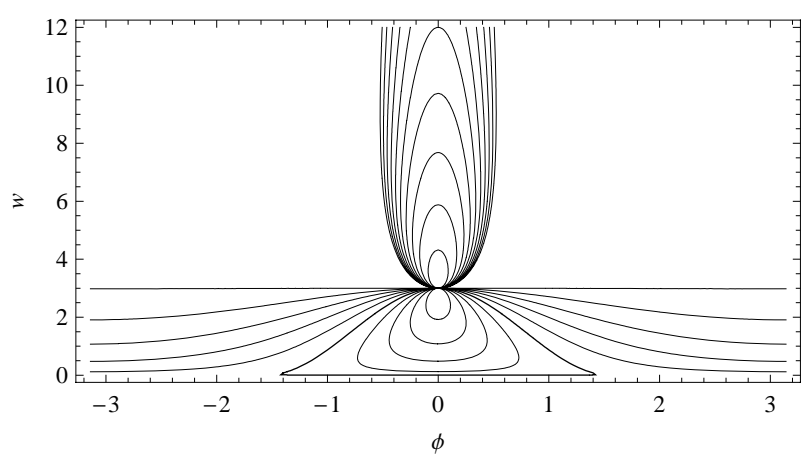

Fig. 1. $H_{1}$-level Hamiltonian contours for $\theta=60^{\circ}$ and $M_{A}^{2} u_{x 0}=1$. In this case $u_{e 0}=u_{p 0}=\tan \theta$ while $u_{x 0}$ takes the values $u_{x 0}=0.0001,0.1(0.1) 2.0$. (and hence $M_{A}$ varies). The horizontal contour corresponds to a $u_{x 0}$ value of 0.0001 . The separatrix (bold curve) is the contour corresponding to $u_{x 0}=0.5$.

the separatrix. The curves in $w<3: u_{x 0}=0.6(0.1) 1.0$ for increasing $u_{x 0}$ correspond to a sequence of closed orbits of decreasing area that converge onto the centre critical point $(\phi, w)=(0,3)$ for $u_{x 0}=1$. Note that all the orbits pass through the same initial point $(\phi, w)=(0,3)$ in the phase plane. The contour for $u_{x 0}=1$ consists of a single isolated point $\left(\phi_{0}, w_{0}\right)=(0,3)$. It is an isolated, centre critical point of $H$. The curves in $w>3$ correspond to $u_{x 0}=1.1(0.1) 2.0$, in which $u_{x 0}$ increases monotonically moving outward from $\phi=0$ in both directions. The tops of the curves are not shown. They consist of a sequence of closed ellipsoidal shaped curves in the region $w \geq 3$, where the topmost points of the curves rise with increasing $u_{x 0}$. Because $M_{A}^{2} u_{x 0}=1$, the Alfvén Mach number of the travelling waves decreases as $u_{x 0}$ increases. Thus, the largest value of $M_{A}$ is the curve $u_{x 0}=0.0001$ for which $M_{A}=100$ and the smallest value of $M_{A}$ is $M_{A}=0.7071$ obtained when $u_{x 0}=2.0$.

Figure 2 shows phase space trajectories $H(\phi, w)=H_{1}$ for $\theta=30^{\circ}, M_{\mathrm{Ae} 0}=0.45, \mu=1836$ in which $u_{x 0}$ is changed in steps of 0.02 from $u_{x 0}=0.0001$ to $u_{x 0}=0.4$. The value of $u_{e 0}$ is fixed by the equation:

$u_{e 0}=\frac{2 u_{x 00}(\mu+1) \tan \theta}{(\mu+1)^{2} / \mu-\tan ^{2} \theta}$

where $u_{x 00}=0.1$. the curve $u_{x 0}=0.1$ corresponds to the separatrix solution and $u_{p 0}$ is chosen to satisfy the integrability constraints Eq. (89). Note that $u_{p 0} \neq u_{e 0}$ in this example (in Fig. $\left.1, u_{e 0}=u_{p 0}=\tan \theta\right)$. In the lower plane $(w<0.014)$, the contours split into two families. The family with $u_{x 0}<0.1$ lies outside the separatrix, with the near horizontal curve corresponding to $u_{x 0}=0.0001$, and $u_{x 0}$ increases monotonically moving downward across the curves until the separatrix $u_{x 0}=0.1$ is obtained. Inside the separatrix $u_{x 0}$, the closed loop contours decrease in size with increasing $u_{x 0}$ until the centre critical point solution is reached for which $u_{x 0}=0.2$.

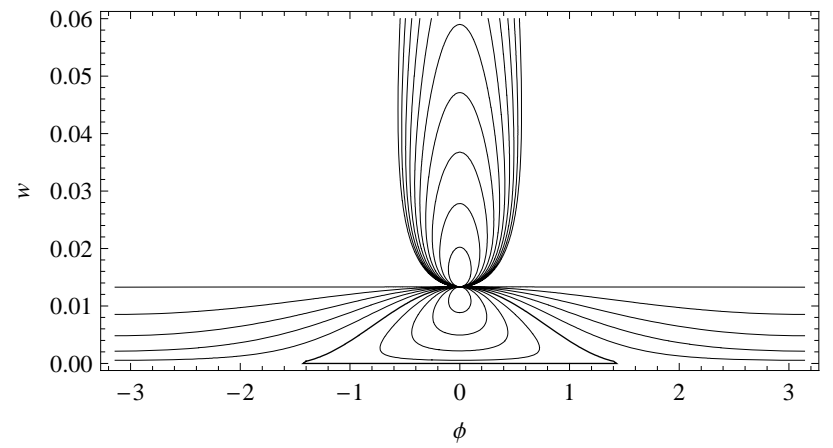

Fig. 2. $H_{1}$-level Hamiltonian contours generated by varying $u_{x 0}$ from 0.02 to 0.4 in steps of 0.02 . The value of $u_{e 0}$ was fixed by evaluating the condition for a separatrix $u_{e 0}=2 u_{x 0}(\mu+1) \tan \theta /\left[(\mu+1)^{2} / \mu-\tan ^{2} \theta\right]$ for the particular case of $u_{x 0}=0.1$. In other words the bell-shaped separatrix-like contour (bold curve) corresponds to the case $u_{x 0}=0.1$. The horizontal contour corresponds to a value of $u_{x 0}$ equal to 0.0001 . Other parameters are: $\theta=30^{\circ}, M_{A e}=0.45$

These contours are bounded in $\phi$ (i.e. $|\phi|<\phi_{m}<\pi / 2$ where $\phi_{m}$ is the maximum value of $\phi$ ). The contours in the upper plane in the region $w>0.014$ are closed curves with $u_{x 0}$ increasing moving upward and outward away from the critical point $\left(u_{x 0}=0.4\right.$ is the outermost curve in $\left.w>0.014\right)$. If one imagines the phase space trajectories $(\phi(x), w(x))$ as wrapped around the surface of a cylinder, where $\phi$ is the azimuthal angle, then the solutions are either: (i) bounded in $|\phi|<\phi_{m}<\pi$ (i.e. the contours inside the separatrix, and in the upper half plane $w>0.014$ ) or (ii) the trajectories stretch from $\phi=-\pi$ to $\phi=\pi$ and wrap around the cylinder in a continuous, periodic fashion as $x$ changes.

The above two examples of Hamiltonian trajectories $H(\phi(x), w(x))=H_{1}$ in the $(\phi, w)$ phase plane illustrate phase trajectories in cases where there is a separatrix. However, the existence of a separatrix depends on whether conditions Eqs. (C5 and C6) in Appendix C are satisfied.

\subsection{The longitudinal structure equation for $u_{x}$}

There are further constraints imposed on the travelling wave solutions that are related to the longitudinal structure Eq. (65) describing the dependence of $u_{x}$ on $x$, which in present case reduces to:

$\frac{d u_{x}}{d x}=-\frac{\sigma(\mu+1) u_{x 0} \cos \theta \sqrt{R\left(u_{x}\right)}}{\mu u_{x}}$,

where $R\left(u_{x}\right)$ is given by Eq. (74) but with $P=u_{x 0} u_{x}, H=H_{1}$, and $\Delta W=0$, namely:

$R\left(u_{x}\right)=\frac{2 \mu\left(M_{A}^{2} u_{x 0}\right) u_{x 0}^{2}}{(\mu+1)^{2}}\left(u_{x}-u_{x 0}\right)\left(u_{x}^{2}+\beta u_{x}+\gamma\right)$, 
where

$$
\begin{aligned}
\beta= & -\frac{1}{2 M_{A}^{2} u_{x 0}}\left(\tan ^{2} \theta+\frac{(\mu+1)^{2}}{\mu}\right), \\
\gamma= & -u_{x 0}^{2}+\frac{u_{x 0}}{2 M_{A}^{2} u_{x 0}}\left(\frac{(\mu+1)^{2}}{\mu}-\tan ^{2} \theta\right) \\
& -\left(\frac{u_{e 0}^{2}+\mu^{2} u_{p 0}^{2}}{2 \mu}\right)+\frac{(\mu+1)^{2}}{2 \mu} \frac{\tan ^{2} \theta}{\left(M_{A}^{2} u_{x 0}\right)^{2}} .
\end{aligned}
$$

Alternatively, $R\left(u_{x}\right)$ can be written in the form:

$R\left(u_{x}\right)=\frac{2 \mu\left(M_{A}^{2} u_{x 0}\right) u_{x 0}^{2}}{(\mu+1)^{2}}\left(u_{x}-u_{x 0}\right)\left(u_{x}-u_{-}\right)\left(u_{x}-u_{+}\right)$,

where the equations:

$$
\begin{aligned}
u_{ \pm}= & \frac{1}{4 M_{A}^{2} u_{x 0}}\left(\tan ^{2} \theta+\frac{(\mu+1)^{2}}{\mu}\right) \pm \sqrt{\Delta} \\
\Delta= & {\left[u_{x 0}-\frac{1}{4 M_{A}^{2} u_{x 0}}\left(\frac{(\mu+1)^{2}}{\mu}-\tan ^{2} \theta\right)\right]^{2} } \\
& +\frac{\left(u_{e 0}-\mu u_{p 0}\right)^{2}}{4 \mu}
\end{aligned}
$$

give the roots $u_{ \pm}$of the quadratic equation $u_{x}^{2}+\beta u_{x}+\gamma=0$.

\subsubsection{Roots of $R\left(u_{x}\right)=0$}

In the case $u_{e 0}=\mu u_{p 0}$, the rotational integral $\mathcal{R}=u_{e 0}^{2}-\mu^{2} u_{p 0}^{2}=0, \Delta$ in Eq. (131) is a perfect square, and the roots $u_{ \pm}$of $R\left(u_{x}\right)=0$ have a simple algebraic form. The analysis of this class of solutions with $\mathcal{R}=0$ is carried out in Appendix D, where we show the connection between these solutions and the Hamiltonian for parallel propagating nonlinear whistler waves investigated by Webb et al. (2005). More general solutions of the longitudinal stucture equation in which $\mathcal{R} \neq 0$ in general, are discussed below.

\section{Proposition 5.6}

The condition for $R\left(u_{x}\right)$ to have a double root, i.e., $R\left(u_{x}\right)=0$ and $R^{\prime}\left(u_{x}\right)=0$ simultaneously, requires

$\left(u_{x}^{2}+\beta u_{x}+\gamma\right)\left(u_{x}-u_{x 0}\right)=0$,

$\left(u_{x}-u_{x 0}\right)\left(2 u_{x}+\beta\right)+u_{x}^{2}+\beta u_{x}+\gamma=0$.

Proof: There are two cases in which Eq. (132) are satisfied simultaneously:

The proof splits into two cases.

Case (i)

If $\Delta=0$ then $u_{+}=u_{-}$is a double root of $R\left(u_{x}\right)=0$. For this root $u_{x}^{2}+\beta u_{x}+\gamma=0$ and $2 u_{x}+\beta=0$, and hence Eq. (132) are satisfied. Note that $\Delta$ is a sum of two squares, and that $\Delta=0$ only if each of the two squares are zero. Thus, the condition $\Delta=0$ is satisfied if

$$
\begin{aligned}
& u_{e 0}=\mu u_{p 0}=\frac{b}{2} \equiv \frac{u_{x 0} \sin \theta \cos \theta}{2 M_{\mathrm{Ae} 0}^{2}}, \\
& M_{\mathrm{Ae} 0}^{2} \sec ^{2} \theta=\frac{\mu+1}{4 \mu}\left(1-\frac{\mu \tan ^{2} \theta}{(\mu+1)^{2}}\right) .
\end{aligned}
$$

The conditions Eqs. (133-134) coupled with the solutions Eq. (131) for $u_{ \pm}$imply

$u_{+}=u_{-}=u_{x 0}\left(1+\frac{\sin ^{2} \theta}{2(\mu+1) M_{\mathrm{Ae} 0}^{2}}\right)=\frac{H_{1}}{u_{x 0}}$.

where $H_{1}$ is the Hamiltonian integral Eq. (87). This solution case corresponds to an oscilliton solution, with zero rotational integral $\mathcal{R}=u_{e}^{2}-\mu^{2} u_{p}^{2}=0$.

Case (ii)

Equations (132) for a double root are also satisfied if:

$u_{x 0}^{2}+\beta u_{x 0}+\gamma=-\frac{D}{\mu}=0$,

where

$$
\begin{aligned}
& D=\mu\left(\frac{\tan ^{2} \theta}{M_{A}^{2}}-u_{e 0} u_{p 0}\right) \equiv \mu\left(\frac{u_{x 0}^{2} \sin ^{2} \theta}{(\mu+1) M_{\mathrm{Ae} 0}^{2}}-u_{e 0} u_{p 0}\right) \\
& \equiv u_{e 0}^{2}-\frac{u_{x 0} \sin \theta \cos \theta}{M_{\mathrm{Ae} 0}^{2}} u_{e 0}+\frac{\mu \sin ^{2} \theta}{(\mu+1) M_{\mathrm{Ae} 0}^{2}} u_{x 0}^{2} .
\end{aligned}
$$

Thus, in this case, the condition for $R\left(u_{x}\right)$ to have a double root, $D=0$, is equivalent to the condition that the integrable solution has a stationary critical point (see Proposition 5.5).

\section{Proposition 5.7}

The roots $u_{x}=u_{ \pm}$of $R\left(u_{x}\right)$ in Eq. (131) may be expressed in the form:

$u_{ \pm}=\frac{u_{*}^{2} \pm\left[\left(u_{*}^{2}-u_{x 0}^{2}\right)^{2}+D u_{x 0}^{2} / \mu\right]^{1 / 2}}{u_{x 0}}$,

where

$$
\begin{aligned}
u_{*}^{2} & =\frac{1}{4 M_{A}^{2}}\left(\tan ^{2} \theta+\frac{(\mu+1)^{2}}{\mu}\right) \\
& \equiv \frac{(\mu+1) u_{x 0}^{2}}{4 \mu M_{\mathrm{Ae} 0}^{2} \sec ^{2} \theta}\left(1+\frac{\mu \tan ^{2} \theta}{(\mu+1)^{2}}\right) .
\end{aligned}
$$

Proof: The proof of Eq. (138) follows from the identity:

$$
\begin{aligned}
& {\left[\frac{1}{4 M_{A}^{2} u_{x 0}}\left(\tan ^{2} \theta+\frac{(\mu+1)^{2}}{\mu}\right)-u_{x 0}\right]^{2}-\Delta} \\
& =u_{p 0} u_{e 0}-\frac{\tan ^{2} \theta}{M_{A}^{2}} \equiv-\frac{D}{\mu},
\end{aligned}
$$

and the formulae Eq. (131) for $u_{ \pm}$. 
Comment: 1

The parameters $\delta_{1}$ of Eq. (118) and $u_{*}$ of Eq.( 139) are related by the equations:

$$
\begin{aligned}
& u_{*}^{2}=u_{x 0}^{2}+\frac{(\mu+1) u_{x 0}^{2}}{4 M_{\mathrm{Ae} 0}^{2} \mu \sec ^{2} \theta}\left(\delta_{1}+\frac{\mu \tan ^{2} \theta}{(\mu+1)^{2}}\right), \\
& \delta_{1}=\frac{u_{*}^{2}-u_{x 0}^{2}\left(1+\mu \tan ^{2} \theta /(\mu+1)^{2}\right)}{u_{*}^{2}} .
\end{aligned}
$$

If $\delta_{1}>0$ (i.e. $\left.M_{\mathrm{Ae} 0}^{2} \sec ^{2} \theta<(\mu+1) / 4 \mu\right)$ then the roots $u_{e 0}=u_{e 0}^{ \pm}$of $D=0$ are real.

Comment: 2

If $D=0$ and $u_{*}^{2}>u_{x 0}^{2}$ then $u_{-}=u_{x 0}$ is a double root of $R\left(u_{x}\right)=0$. If $u_{*}^{2}<u_{x 0}^{2}$ then $u_{+}=u_{x 0}$. However, this latter case does not lead to physical solutions, because real solutions for $u_{e 0}$ do not exist in this case.

The physically allowed solutions for $u_{x}$ as a function of $x$ in Eq. (126) requires that $R\left(u_{x}\right) \geq 0$, and that the solution pass through $u_{x}=u_{x 0}$. In addition for integrable solutions, $u_{e 0}$ and $u_{p 0}$ must satisfy Eq. (89), and $u_{e 0}$ and $u_{p 0}$ must be real and non-negative. The nature of the integrable solutions for $u_{x}$ depends on the initial data for $u_{x 0}, u_{e}$ and $u_{p 0}$, which in turn depend on whether (i) $D>0$, (ii) $D<0$ or (iii) $D=0$, where $D\left(u_{e 0}\right)$ is given by Eq. (137).

\section{Proposition 5.8}

The physically allowed solutions for $u_{x}$ versus $x$, for given initial data for $u_{x 0}, u_{e 0}$ and $u_{p 0}$ requires $R\left(u_{x}\right) \geq 0$, which depends on whether (i) $D>0$, (ii) $D<0$ or (iii) $D=0$. The different possible solution cases are:

(i) Case $D>0$

In this case $R\left(u_{x}\right) \geq 0$ for $u_{x}$ in the range $u_{-} \leq u_{x} \leq u_{x 0}<u_{+}$. If $\delta_{1}>0$, then $u_{e 0}$ must be chosen to lie in the range:

$$
\left\{0 \leq u_{e 0} \leq u_{e 0}^{-}\right\} \cup\left\{u_{e 0}^{+} \leq u_{e 0} \leq \frac{u_{x 0} \sin \theta \cos \theta}{M_{\mathrm{Ae} 0}^{2}}\right\}
$$

If $\delta_{1}<0$ then $u_{e 0}$ must be chosen in the range:

$$
\left\{0 \leq u_{e 0} \leq \frac{u_{x 0} \sin \theta \cos \theta}{M_{\mathrm{Ae} 0}^{2}}\right\} \text {. }
$$

$u_{p 0}$ is obtained by using the integrability constraint Eq. (89):

$\mu u_{p 0}=\frac{u_{x 0} \sin \theta \cos \theta}{M_{\mathrm{Ae} 0}^{2}}-u_{e 0}$.

(ii) Case $D<0$

In this case, the relative location of the roots depends on whether $u_{*}^{2}>u_{x 0}^{2}$ or $u_{*}^{2}<u_{x 0}^{2}$. If $u_{*}^{2}>u_{x 0}^{2}$ and $\delta_{1}>0$ then $R\left(u_{x}\right) \geq 0$ for $u_{x}$ in the range: $u_{x 0} \leq u_{x} \leq u_{-}<u_{+}$and $u_{e}$ must be chosen in the range

$u_{e 0}^{-} \leq u_{e 0} \leq u_{e 0}^{+}$, and $\mu u_{p 0}$ is given by Eq. (144). Note that $\delta_{1}>0$, using Eq. (141) implies $u_{*}^{2}>u_{x 0}^{2}$. If $u_{*}^{2}<u_{x 0}^{2}$, then $\delta_{1}<-\mu \tan ^{2} \theta /(\mu+1)^{2}$ in Eq. (141), which leads to the contradiction $D>0$. Hence, there are no exact solutions if $u_{*}^{2}<u_{x 0}^{2}$.

(iii) Case $D=0$

In this case the roots Eq. (138) for $u_{ \pm}$reduce to:

$u_{ \pm}=\frac{u_{*}^{2} \pm\left|u_{*}^{2}-u_{x 0}^{2}\right|}{u_{x 0}}$

If $u_{*}^{2}>u_{x 0}^{2}$ then $u_{x}=u_{-}=u_{x 0}$ is a double root of $R\left(u_{x}\right)$ and

$u_{+}=\frac{2 u_{*}^{2}-u_{x 0}^{2}}{u_{x 0}}>u_{x 0}$.

A sketch of $R\left(u_{x}\right)$ versus $u_{x}$ reveals that the only solution possible is the isolated point $u_{x}=u_{x 0}$. We also require $\delta_{1} \geq 0$ in order that $u_{e 0}$ and $u_{p 0}$ are real. In fact

$u_{e 0}=u_{e 0}^{ \pm}$,

are the only values of $u_{e 0}$ that are allowed, and $\mu u_{p 0}$ is given in terms of $u_{e 0}$ by Eq. (144). If $u_{*}^{2}<u_{x 0}^{2}$ then $\delta_{1}<0, u_{e 0}^{ \pm}$are complex, and hence there are no solutions in this case.

Comment:

In addition to the above constraints on $u_{x}$, we require $u_{e}^{2}>0$, which from Eq. (103) requires:

$u_{x}^{2} \leq u_{x 0}^{2}+\frac{u_{e 0}^{2}}{\mu}$

in order that $u_{e}$ is real.

\subsubsection{Integration of the longitudinal structure equation}

The longitudinal structure Eq. (126) can be integrated to give $x$ as a function of $u_{x}$ in the wave as:

$x=-\frac{\mu}{\sqrt{2 \mu M_{A}^{2} u_{x 0}} u_{x 0}^{2} \cos \theta} \int_{u_{1}}^{u_{x}} \frac{\sigma u_{x}^{\prime}}{\sqrt{Q\left(u_{x}^{\prime}\right)}} d u_{x}^{\prime}$,

where

$Q\left(u_{x}\right)=\left(u_{x}-u_{1}\right)\left(u_{x}-u_{2}\right)\left(u_{x}-u_{3}\right)$.

where $u_{1}<u_{2}<u_{3}$ are the roots of $R\left(u_{x}\right)=0$ in increasing order. From Eqs. (130-131):

$u_{1}=\min \left(u_{x 0}, u_{-}\right), \quad u_{2}=\max \left(u_{x 0}, u_{-}\right), \quad u_{3}=u_{+}$.

Thus, for Case (i) $u_{1}=u_{-}$and $u_{2}=u_{x 0}$, but for Case (ii), $u_{1}=u_{x 0}$ and $u_{2}=u_{-}$. In Eq. (150) we have chosen the integration constant so that $x=0$ corresponds to the point where $u_{x}=u_{1}$. Note we require $u_{1} \leq u_{x} \leq u_{2}$ in order that $Q\left(u_{x}\right)>0$ for a physical real solution for $x$. 
Using the change of integration variable:

$z=\left(\frac{u_{x}^{\prime}-u_{1}}{u_{2}-u_{1}}\right)^{1 / 2}$

the integral Eq. (150) can be reduced to a combination of elliptic integrals of the form:

$$
\begin{gathered}
x=-\frac{\sigma \sqrt{2 \mu}}{u_{x 0}^{2} \cos \theta \sqrt{M_{A}^{2} u_{x 0}} \sqrt{u_{3}-u_{1}}} \\
\left(u_{3} U-\left(u_{3}-u_{1}\right) E(U \mid v)\right),
\end{gathered}
$$

where

$$
\begin{aligned}
U & =\int_{0}^{y} \frac{d z}{\sqrt{\left(1-z^{2}\right)\left(1-v z^{2}\right)}} \\
& \equiv \int_{0}^{\varphi} \frac{d \varphi^{\prime}}{\sqrt{1-v \sin ^{2} \varphi^{\prime}}}, \\
E(U \mid v) & =\int_{0}^{y}\left(\frac{1-v z^{2}}{1-z^{2}}\right)^{1 / 2} d z \\
& \equiv \int_{0}^{\varphi} \sqrt{1-v \sin ^{2} \varphi^{\prime}} d \varphi^{\prime}, \\
y & =\left(\frac{u_{x}-u_{1}}{u_{2}-u_{1}}\right)^{1 / 2}=\operatorname{sn}(U \mid v)=\sin (\varphi), \\
v & =\frac{u_{2}-u_{1}}{u_{3}-u_{1}} .
\end{aligned}
$$

Here $U=\mathrm{sn}^{-1}(y \mid v)$ and $E(U \mid v)$ are standard elliptic integrals of the first and second kind (Abramowitz and Stegun, 1965, Ch. 17, p. 589). The incomplete elliptic integral of the second kind $E(U \mid v)$ can be expressed in a variety of different forms (e.g. Abramowitz and Stegun, 1965, Ch. 17).

Note that Eq. (131) pertains to a range of $u_{x}$ where $\sigma=\operatorname{sgn}(\sin \phi)$ is constant. It is straightforward to modify Eq. (154) to account for the change of sign of $\sin \phi$ throughout the wave.

By noting that $y=0$ when $u_{x}=u_{1}$ and $y=1$ when $u_{x}=u_{2}$ it follows from Eq. (150) that the spatial period of the wave, $L$, is given by

$$
\begin{gathered}
L=\frac{2 \sqrt{2 \mu}}{u_{x 0}^{2} \cos \theta \sqrt{M_{A}^{2} u_{x 0}} \sqrt{u_{3}-u_{1}}} \\
{\left[u_{3} K-\left(u_{3}-u_{1}\right) E\right],} \\
K=K(v)=\int_{0}^{1} \frac{d z}{\sqrt{\left(1-z^{2}\right)\left(1-v z^{2}\right)}}, \\
E=E(K \mid v)=\int_{0}^{1}\left(\frac{1-v z^{2}}{1-z^{2}}\right)^{1 / 2} d z,
\end{gathered}
$$

where $K(v)$ and $E(K \mid v)$ are complete elliptic integrals of the first and second kind.

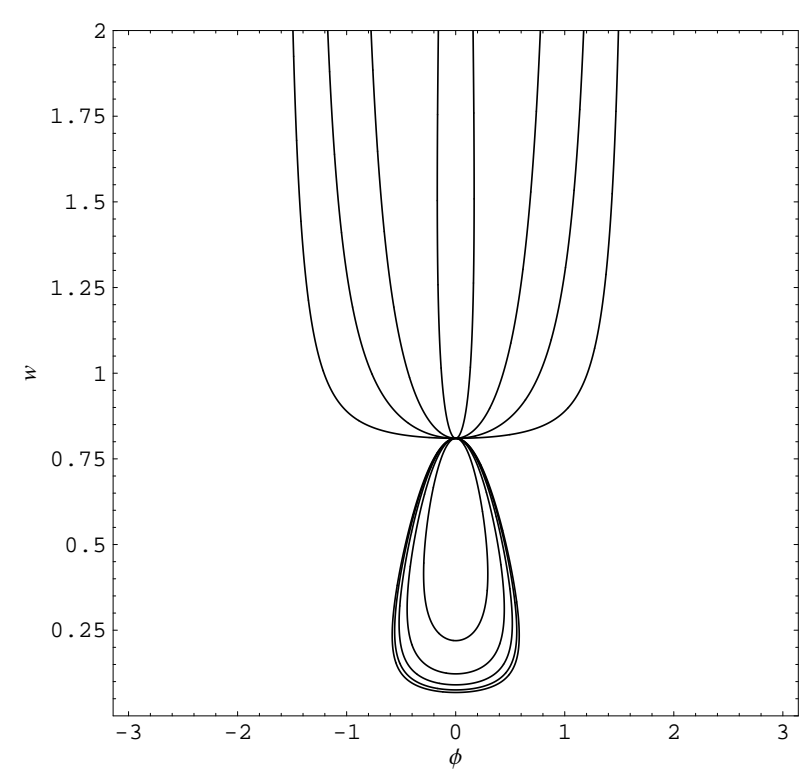

Fig. 3. Contours of the Hamiltonian $H=H_{1}$ (Eqs. 81 and 87) for $u_{e 0}=0.9$ and a range of $M_{\mathrm{Ae} 0}$ : $0.1<M_{\mathrm{Ae} 0}<\left(u_{x 0} \sin \theta \cos \theta / u_{e 0}\right)^{1 / 2} . \theta=30^{\circ}, u_{x 0}=1$ and $\mu=1836$. There are two different classes of solutions: one with $u_{e 0}^{-}<u_{e 0}$ (contours in the lower part of the figure where $u_{e}<u_{e 0}=0.9$ ) and another with $u_{e 0}<u_{e 0}^{-}$(contours in the upper part of the figure where $\left.u_{e}>u_{e 0}=0.9\right)$. The upper contours are closed curves whose apexes extend to large values of $u_{e}^{2}$ (not shown).

\subsection{Examples}

In the cold plasma, travelling wave examples below, we use, in the main, the following fixed parameters: $\theta=30^{\circ}$, $\mu=1836$ and $u_{x 0}=1$. The solutions are obtained by integrating the transverse electron and proton momentum Eqs. (6) with $\delta_{0}=0$, and by using the rotational integral $\mathcal{R}$ and energy integral in the dHT frame $\varepsilon_{H T}$, to express $u_{x}$ in terms of $u_{e}$, i.e.,

$u_{x}=\left[u_{x 0}^{2}+\left(u_{e 0}^{2}-u_{e}^{2}\right) / \mu\right]^{1 / 2}$,

Note that the constant term in Eq. (161) can be expressed in terms of $\mathcal{R}$ and $\varepsilon_{H T}$ (see Eqs. 71 and 72). The initial conditions used in the numerical integration of Eq. (6) are those in Eq. (89), i.e.,

$\phi_{e 0}=\phi_{p 0}=\pi / 2, \quad u_{e 0}+\mu u_{p 0}=b \equiv \frac{u_{x 0} \sin \theta \cos \theta}{M_{\mathrm{Ae} 0}^{2}}$,

which ensures that the total, transverse momentum fluxes in the dHT frame are zero (i.e. $\delta_{0}=0$ ). The initial data arise from imposing a particular choice of the initial phases, namely $\phi_{e 0}=\phi_{p 0}=\pi / 2$ in the more general integrability conditions Eq. (86). More general solutions of Eq. (86) (i.e., the 

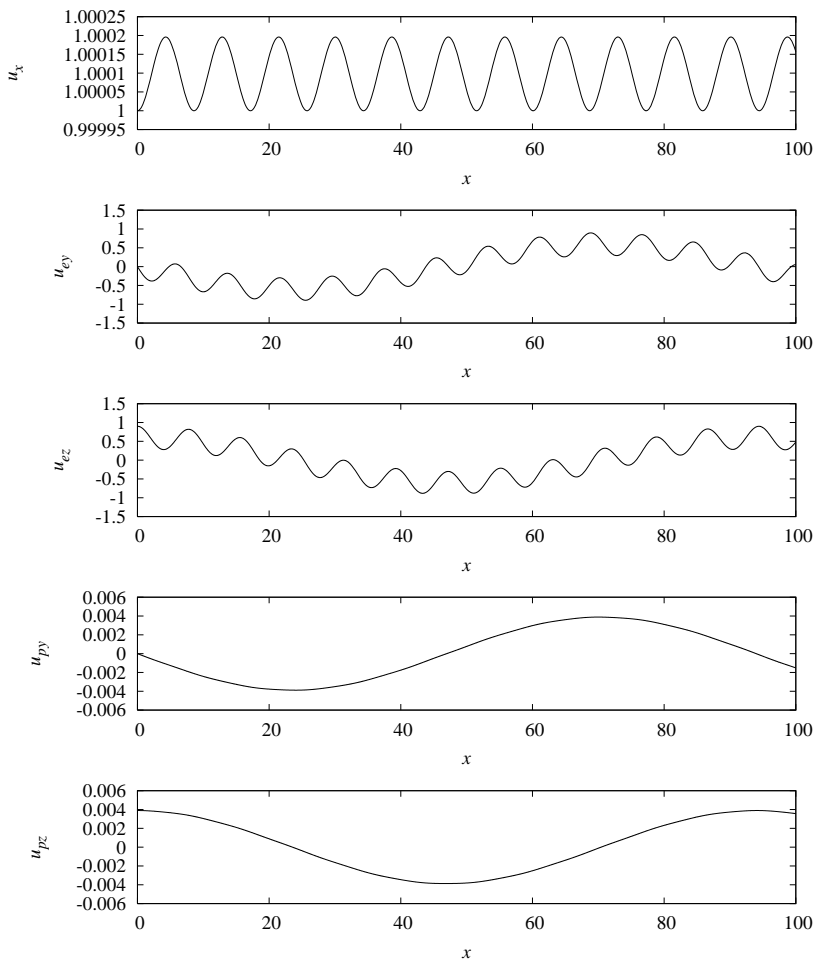

Fig. 4. Velocity components as a function of $x$ for the $H_{1}$-level curve with $M_{\mathrm{Ae} 0}=0.231918$ in Fig. 3 (all other parameters as given in Fig. 3). This is the third curve moving inwards in $w<0.81$ in that figure and it corresponds to a negative value of the rotational integral $\mathcal{R}=-50.32$.

Eqs. $\left.\delta_{0}=0\right)$ are discussed in Appendix A. Note that the initial data Eq. (162) imply that $u_{e y 0}=u_{p y 0}=0$ and that $u_{e z 0} \equiv u_{e 0}$ and $u_{p z 0} \equiv u_{p 0}$ are both non-negative. From Eq. (162) it follows that

$0 \leq u_{e 0} \leq b \quad$ and $\quad 0 \leq \mu u_{p 0} \leq b$.

The choice Eq. (162) does allow for the possibility of parallel propagating waves, but only for the case $u_{e 0}=u_{p 0}=0$. In other words, it rules out a large number of physical solutions that are possible in this case (see Appendix A for other possible cases). The condition Eq. (163) for $u_{e 0}$ implies the restriction to oblique travelling waves with:

$\theta \geq \theta_{c}=\frac{1}{2} \arcsin \left(2 M_{\mathrm{Ae} 0}^{2} \frac{u_{e 0}}{u_{x 0}}\right)$,

(note $u_{e 0}$ can also depend on $\theta$ ). One can investigate the case of quasi-parallel propagating waves with $0<\theta<\theta_{c}$ by using other initial data described in Appendix A (e.g. the solutions of $\delta_{0}=0$ given in Eq. A11 and A12). However, in the present paper, we restrict our attention to solutions satisfying the initial data Eq. (162). The numerical accuracy of the solutions was monitored by checking the constancy of the known integrals $\varepsilon_{H T}, \mathcal{R}$ and $H_{1}$ during the computations.

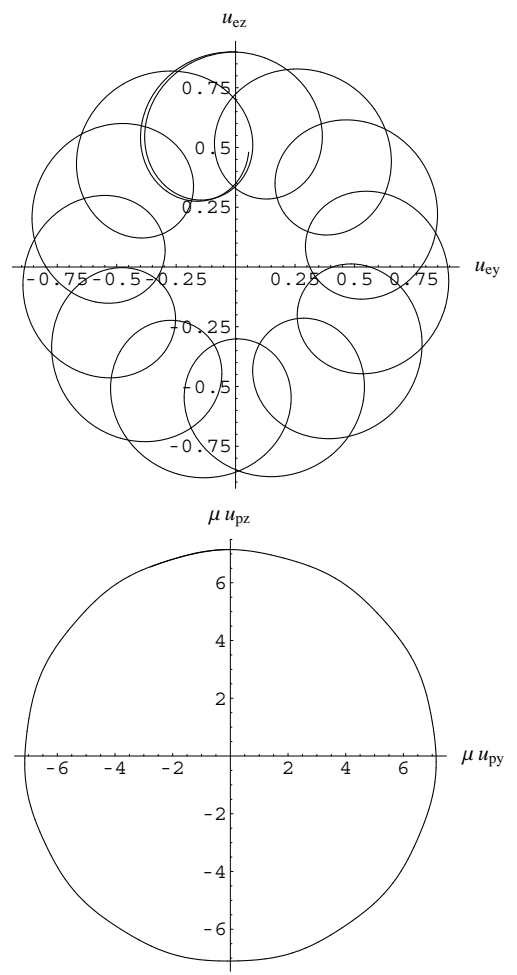

Fig. 5. Electron (upper panel) and proton (lower panel) perpendicular velocity "hodographs" for the $H_{1}$-level curve with $M_{\mathrm{Ae} 0}=0.231918$ in Fig. 3 (all other parameters as given in Fig. 3). This is the third curve moving inwards in that figure $(w<0.81)$ corresponding to a rotational integral $\mathcal{R}=-50.32$. Same parameter values as in Fig. 4.

Figure 3 shows the $(\phi, w)$ phase space contours $H=H_{1}$, of a sequence of different Hamiltonians, obtained by varying $M_{\mathrm{Ae} 0}$, and with $u_{e 0}=0.9, \theta=30^{\circ}, u_{x 0}=1$ and $\mu=1836$ fixed. The contours represent two distinct types of solution, distinguished by whether $u_{e 0}<u_{e 0}^{-}$or $u_{e 0}>u_{e 0}^{-}$, where $u_{e 0}^{-}$is the smaller root of $D\left(u_{e 0}\right)=0$. Note that $u_{e 0}^{-}$varies as $M_{\mathrm{Ae} 0}$ changes. For the lower, tear shaped contours in $w<0.81$, $D\left(u_{e 0}\right)<0$ and it is necessary that $u_{e 0}$ lie in the range $u_{e 0}^{-}<u_{e 0}<u_{e 0}^{+}$and $u_{x 0}<u_{x}<u_{-}$for the physically allowed solutions. For the upper sequence of contours, $D\left(u_{e 0}\right)>0$. If $D\left(u_{e 0}\right)>0$ then it is necessary that either $u_{e 0}<u_{e 0}^{-}$or $u_{e 0}>u_{e 0}^{+}$if $\delta_{1}>0\left(\delta_{1}=0\right.$ when $M_{\mathrm{Ae} 0}=0.4333$ in the present example). However, if $\delta_{1}<0$, then $u_{e 0}$ must lie in the range $0<u_{e 0}<b$. The values of $u_{x}$ lie in the range $u_{-}<u_{x}<u_{x 0}$ for the upper contours. For the examples in Fig. 3, $u_{e 0}<u_{e 0}^{-}$ for the upper contours. For the isolated centre critical point $\left(\phi_{0}, w_{0}\right)=(0,0.81), D\left(u_{e 0}\right)=0$ and $u_{x 0}=u_{-}$is a double root of $R\left(u_{x}\right)=0$. The transition from the lower $(w<0.81)$ to the upper curves $w>u_{e 0}^{2}=0.81$ occurs when $D\left(u_{e 0}\right)=0$ (in fact when $\left.u_{e 0}=u_{e 0}^{-}\right)$. Using Eq. (115) for $D\left(u_{e 0}\right)$, and setting $D\left(u_{e 0}\right)=0$, we obtain an equation for $M_{\mathrm{Ae} 0}^{2}$ where the curves transit from the lower to the upper sequence of contours, 

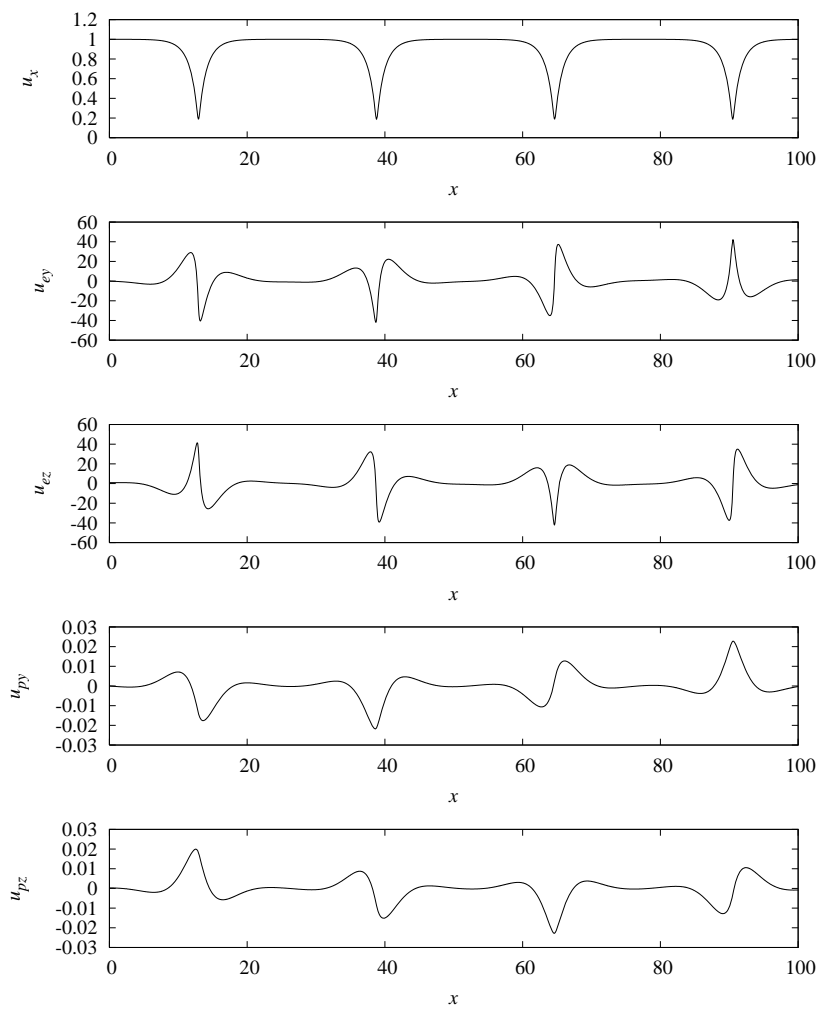

Fig. 6. Velocity components as a function of $x$ for the $H_{1}$-level curve with $M_{\mathrm{Ae} 0}=0.561714$ in Fig. 3 (all other parameters as given in Fig. 3). This is the second curve moving inwards in the $w>0.81$ part of that figure and it corresponds to a positive value of the rotational integral $\mathcal{R}=0.586868$.

namely when

$M_{\mathrm{Ae} 0}^{2}=\frac{u_{x 0} \sin \theta}{u_{e 0}^{2}}\left(u_{e 0} \cos \theta-\frac{\mu \sin \theta}{\mu+1} u_{x 0}\right)$.

This corresponds to $M_{\mathrm{Ae} 0}^{2}=0.17247$ or $M_{\mathrm{Ae} 0}=0.415294$. At this value of $M_{\mathrm{Ae} 0}, u_{-}=u_{x} 0$ is a double root of $R\left(u_{x}\right)=0$, and the contour consists of the centre critical point $\left(\phi_{0}, w_{0}\right)=(0,0.81)$. On the upper branch $w>0.81, \delta_{1}>0$ for low Mach numbers: $0.415294<M_{\mathrm{Ae} 0}<0.4333, \delta_{1}=0$ when $M_{\mathrm{Ae} 0}=0.4333$, and $\delta_{1}<0$ for larger values of $M_{\mathrm{Ae} 0}$. The largest value of $M_{\mathrm{Ae} 0}$ allowed occurs when $u_{e 0}=b$, which from Eq. (162) occurs when

$M_{\mathrm{Ae} 0}^{2}=\frac{u_{x 0} \sin \theta \cos \theta}{u_{e 0}}=0.4811 \quad$ or $\quad M_{\mathrm{Ae} 0}=0.6936$.

It should be noted that only a portion of the upper contours is shown, in order to emphasize the contour structure in the vicinity of $\left(\phi_{0}, w_{0}\right)=(0,0.81)$. The upper contours form closed curves, which begin at the right hand side of the figure and return on the left hand side. The top of the curves
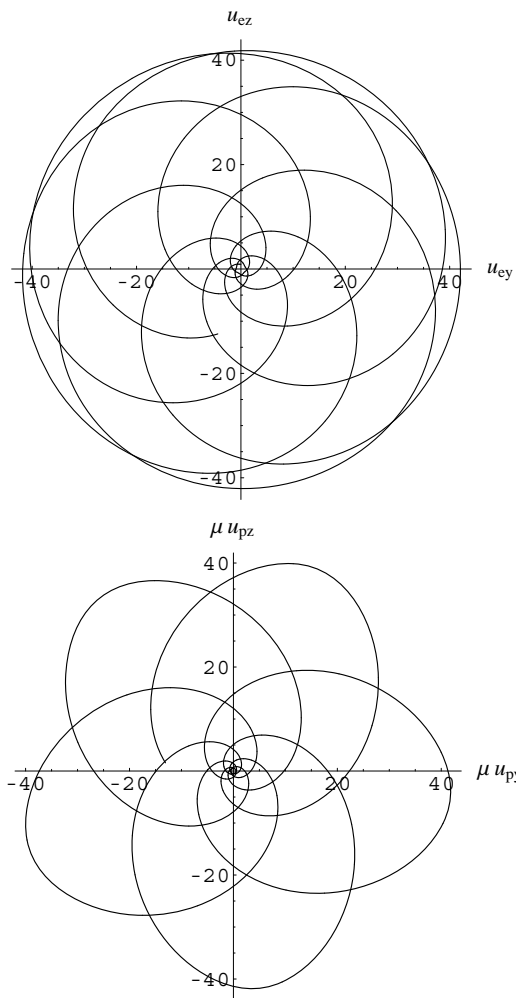

Fig. 7. Electron (upper panel) and proton (lower panel) perpendicular velocity "hodographs" for the $H_{1}$-level curve with $M_{\mathrm{Ae} 0}=0.561714$ in Fig. 3 (all other parameters as given in Fig. 3). This is the second curve moving inwards in the $w>0.81$ part of that figure, corresponding to a rotational integral $\mathcal{R}=0.586868$.

in $w>0.81$ occur when $u_{x}=u_{-}$. For the lower contours ( $w<0.81$ ), the bottom of the loops correspond to $u_{x}=u_{-}$.

The behaviour of the velocity components of the electrons and protons for representative cases are shown in Figs. 45 and Figs. 6-7. Figures 4-5 show an example for which $w<0.81$ and $M_{\mathrm{Ae} 0}=0.231918$ (it corresponds to the third $H_{1}$ level curve moving inwards in Fig. 3). For this solution $u_{e 0}>u_{e 0}^{-}$, and the rotational integral $\mathcal{R}=-50.32$. The fluid velocity variations in this example are predominantly in the transverse directions, with very little variation in the longitudinal velocity $u_{x}$ (this is reminiscent of the Alfvén wave in MHD, which is an incompressible mode, in which there are no variations in $u_{x}$ : e.g. Chen, 1984, p. 136 et seq., Webb et al., 1996). The transverse velocities of the electrons consist of a long scale variation, on which are superimposed finer scale variations, whilst the protons exhibit only a long scale variation. This difference in the behaviour of the protons and electrons, is more vividly illustrated in Fig. 5 which show hodograph type plots of the transverse velocity components of the electrons (top panel) and the protons (lower panel). It is seen that the protons execute an almost circular, smooth rotational motion in the $\left(\mu u_{p y}, \mu u_{p z}\right)$ plane, but the electrons 


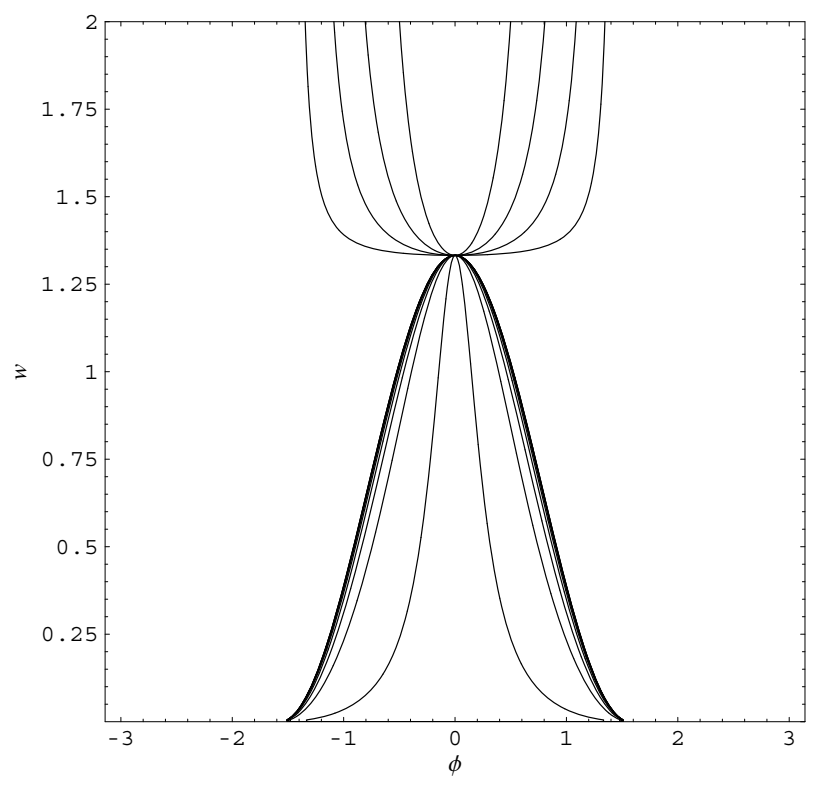

Fig. 8. Contours of the Hamiltonian $H=H_{1}$ for fixed $u_{e 0}=2 u_{x 0}(\mu+1) \tan \theta /\left((\mu+1)^{2} / \mu-\tan ^{2} \theta\right)$ and a sequence of $M_{\mathrm{Ae} 0}$ values beginning at 0.1 and ending at $\left(u_{x 0} \sin \theta \cos \theta / u_{e 0}\right)^{1 / 2}$ (note that this represents a sequence of different Hamiltonian functions). $\theta=30^{\circ}, u_{x 0}=1$ and $\mu=1836$. The lower sequence with $u_{e 0}^{-}<u_{e 0}$ correspond to separatrix-type solutions.

execute a rosette type motion, in which the orbit precesses in a circular type pattern. It turns out that the type of motion of the protons and the electrons in the transverse plane, depends very much on the value of the rotational integral $\mathcal{R}$ (in this example, $\mathcal{R}$ is large and negative).

Figures 6 and 7 show the electron and proton velocities for a travelling wave with $M_{\mathrm{Ae} 0}=0.56174$. This example corresponds to the second $H_{1}$ contour, moving inward, in the region $w>0.81$ of the $(\phi, w)$ phase-plane in Fig. 3 . The rotational integral $\mathcal{R}=0.586868$ in this example. The profile for $u_{x}$ shows a long flat section with $u_{x} \approx 1$, followed by a fast deceleration and acceleration phase. The transverse electron and proton fluid velocities $\left(u_{j y}, u_{j z}\right)(j=e, p)$ also show a long flat section, where $u_{e} \approx 0$ and $u_{p} \approx 0$, followed by a fast spatial evolutionary phase. Thus, the wave exhibits both a slowly varying phase, followed by a rapid evolutionary phase. Figure 7 shows the transverse electron and proton velocity hodographs: $u_{e z}$ versus $u_{e y}$ (upper panel) and $\mu u_{p z}$ versus $\mu u_{p y}$ (bottom panel). Both the protons and the electrons exhibit complex rosette patterns, that resemble precessing ellipses. The slow and fast evolutionary phases are reflected in the hodographs in Fig. 7, where the electrons spend a large part of their time in the vicinity of $u_{e} \approx 0$. This is followed by a fast acceleration to larger $u_{e}$, followed by a deceleration back to $u_{e} \approx 0$, which appears as a loop on the hodograph plot. A similar pattern occurs for the protons.
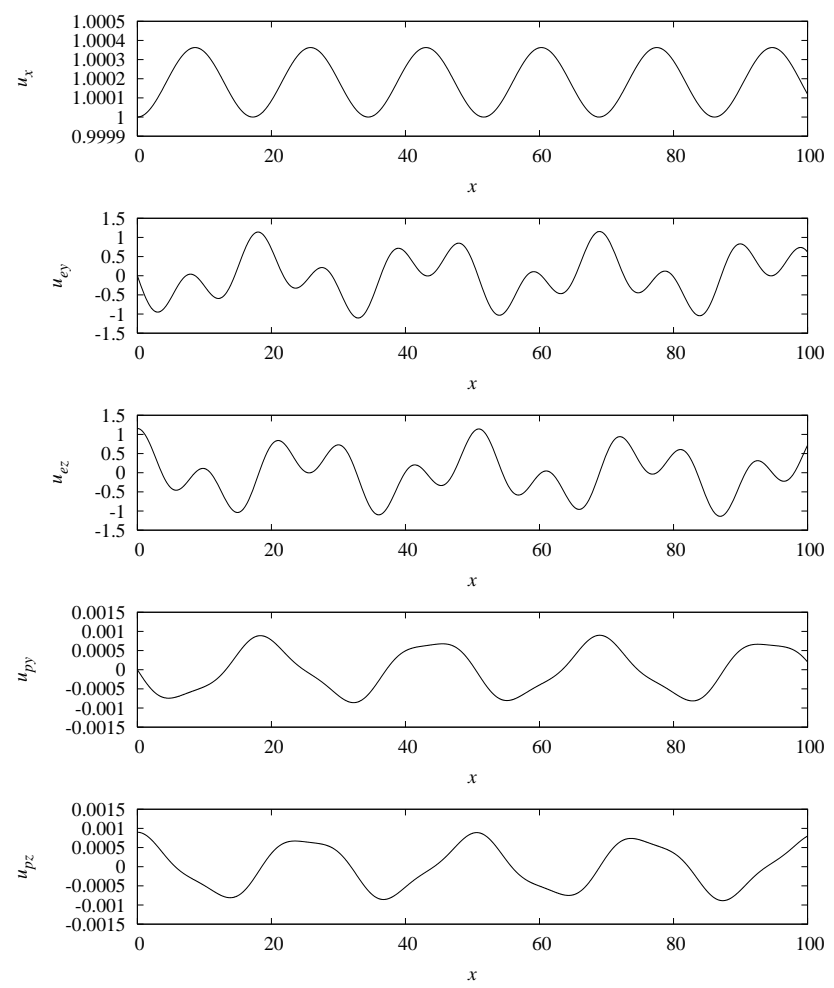

Fig. 9. Velocity components as a function of $x$ for the $H_{1}$-level curve with $M_{\mathrm{Ae} 0}=0.392848$ in Fig. 8 . This is the second curve moving outwards in the lower part of that figure and it corresponds to a rotational integral of $\mathcal{R}=-1.39505$.

Figure 8 shows contours of the Hamiltonian $H=H_{1}$ in the $(\phi, w)$ phase plane similar to the plots in Fig. 3, except that $u_{e 0}$ is now determined from the condition:

$u_{e 0}=\frac{2 u_{x 0}(\mu+1) \tan \theta}{\left[(\mu+1)^{2} / \mu-\tan ^{2} \theta\right]}$,

which gives separatrix type solutions if $D\left(u_{e 0}\right)<0$ and $u_{e 0}^{-}<u_{e 0}<u_{e 0}^{+}$. These solutions pass through the nonstandard critical points at $(\phi, w)=( \pm \pi / 2,0)$. As $M_{\mathrm{Ae} 0}$ increases, $u_{e 0}^{-}$increases and approaches $u_{e 0}$ until $u_{e 0}^{-}=u_{e 0}$, at which point $D\left(u_{e 0}\right) \equiv D\left(u_{e 0}^{-}\right)=0$. At this value of $M_{\mathrm{Ae} 0}$, $M_{\mathrm{Ae} 0}=M_{c}$, the contour consist of a single point, the centre critical point. For larger $M_{\mathrm{Ae} 0}$, there are no separatrices, and the contours form loops in the upper portion of the $(\phi, w)$ plane where $w>u_{e 0}^{2}$. By differentiation of $D\left(u_{e 0}\right)=0$ with respect to $M_{\mathrm{Ae} 0}^{2}$, we obtain

$\frac{d u_{e 0}}{d M_{\mathrm{Ae} 0}^{2}}=\frac{u_{e 0}^{2}}{b \sqrt{\delta_{1}} M_{\mathrm{Ae} 0}^{2}}$,

which shows that $u_{e 0}^{ \pm}$both increase with increasing $M_{\mathrm{Ae} 0}^{2}$. In Fig. 8, $M_{\mathrm{Ae} 0}$ varies from 0.1 up to $\left(u_{x 0} \sin \theta \cos \theta / u_{e 0}\right)^{1 / 2}$.

Figure 9 shows the fluid velocity components of the electrons and protons for a separatrix type solution with $M_{\mathrm{Ae} 0}=$ 

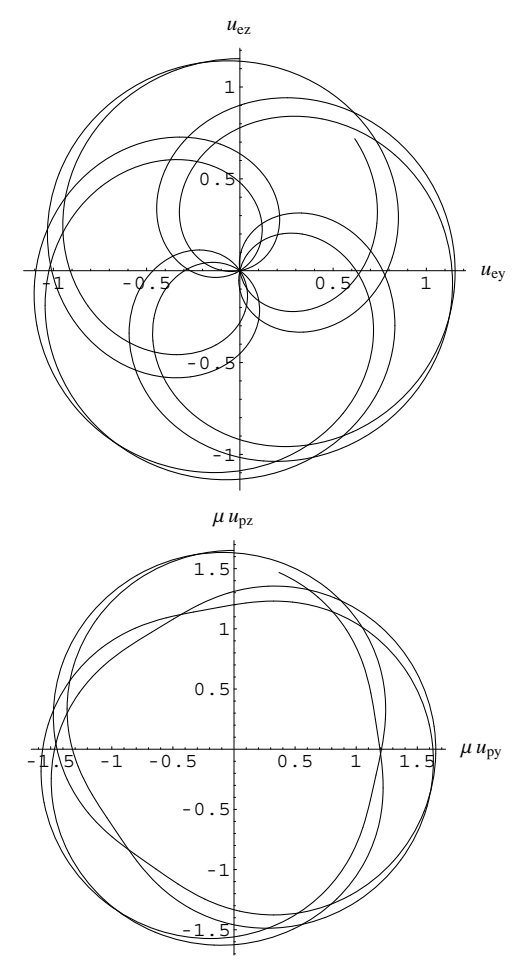

Fig. 10. Electron (upper panel) and proton (lower panel) perpendicular velocity "hodographs" for the $H_{1}$-level curve with $M_{\mathrm{Ae} 0}=0.392848$ in Fig. 8. This is the second curve moving outwards in the lower part of that figure, and corresponds to the plots in Fig. 9. The rotational integral $\mathcal{R}=-1.39505$.

0.392848 , corresponding to the second curve moving outward in the lower portion of Fig. 8. The dynamics are dominated by the transverse velocity variations, and there is only a very small change in $u_{x}$ (note $1<u_{x}<1.0004$ ). The hodographs of the transverse electron and proton velocities in Fig. 10 again show complex rosette patterns. Noteworthy is the fact that the perpendicular electron speed $u_{e}=\left(u_{e y}^{2}+u_{e z}^{2}\right)^{1 / 2}$ periodically passes through zero. At this point, there is a very fast change in $\phi=\phi_{p}-\phi_{e}$, without a significant change in $u_{e}$. Note that this is consistent with the separatrix solution plots in the $(\phi, w)$ phase plane (Fig. 8), because the separatrix near $u_{e} \approx 0$ joins the two non-standard critical points $\left(\phi_{2}, w_{2}\right)=(-\pi / 2,0)$ and $\left(\phi_{3}, w_{3}\right)=(\pi / 2,0)$ where there is a change of $\delta \phi=\pi$ in the value of $\phi$, without any significant change in $u_{e}$.

Comment: To understand the behaviour of the travelling wave solutions illustrated in Figs. 4-10, it is useful to have at hand, the zeros of the function $R\left(u_{x}\right)$ in the longitudinal structure Eq. (126) and to know the zeros of $D\left(u_{e 0}\right)$. Figure 11 illustrates $R\left(u_{x}\right)$ (top panel) and $D\left(u_{e 0}\right)$ (lower panel) corresponding to the solutions in Figs. 4 and 5. The $u_{x}$ variation in the solution is confined to the region $u_{x 0}<u_{x}<u_{-}$ where $R\left(u_{x}\right)>0$. The solution varies periodically between
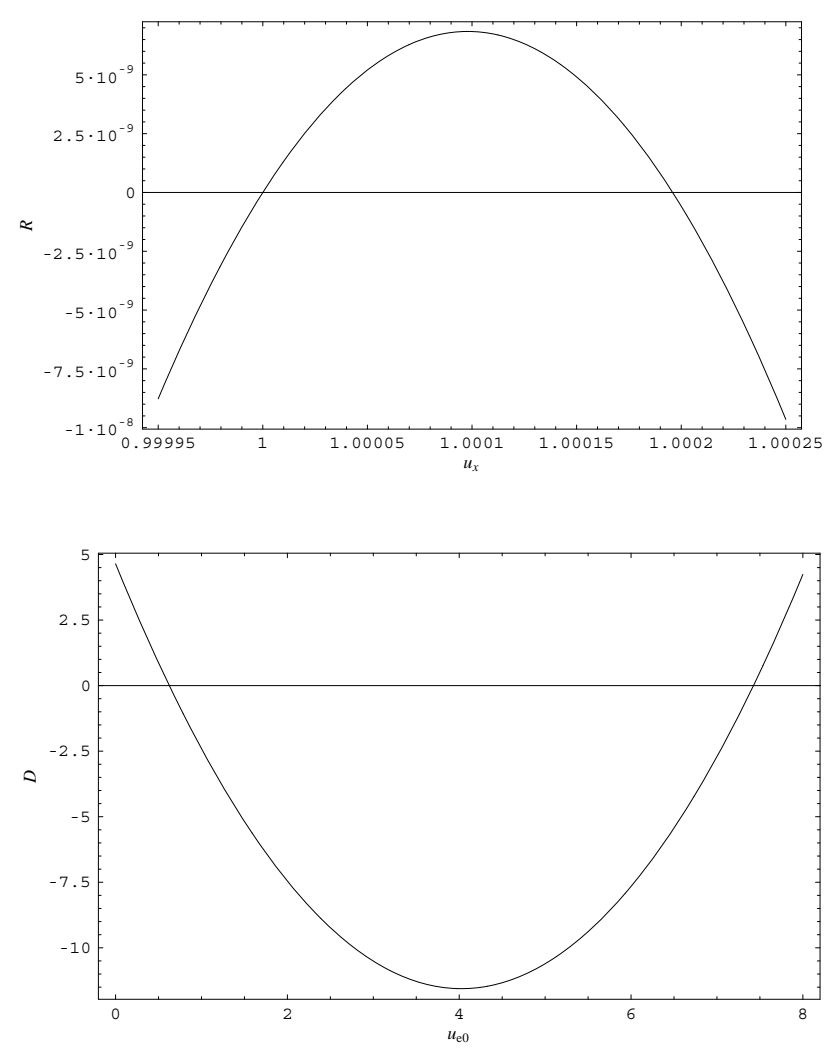

Fig. 11. The functions $R\left(u_{x}\right)$ and $D\left(u_{e 0}\right)$ for the solution shown in Figs. 4 and 5 . The velocity component $u_{x}$ oscillates periodically between its initial value $u_{x 0}=1$ and the root $u-=1.0002$ (see upper panel). The initial value of $u_{e 0}$ is equal to 0.9 , which corresponds to a negative value of $D$ (lower panel).

its initial value $u_{x 0}=1$ and the root $u_{-}=1.0002$. In the numerical solutions, $u_{e 0}=0.9$ and hence $D\left(u_{e 0}\right)<0$. Note the extremely small range of $u_{x}$ for the solution: $1<u_{x}<1.0002$, which implies the need for both accurate computations and rigorous algebraic analysis in order to explore the interesting regions of phase space.

Figures 12 and 13 illustrate a solution example in which $R\left(u_{x}\right)=0$ has a double root $u_{x}=u_{x 0}=u_{-}$, for which $\left(\phi_{0}, w_{0}\right)=\left(0, u_{e 0}^{2}\right)$ is a centre critical point in the $(\phi, w)$ phase plane. The parameters used were $u_{x 0}=0.5$, $M_{\mathrm{Ae} 0}=0.255, \theta=30^{\circ}$ and $u_{e 0}=3.01049$. Note $u_{x}=u_{x 0}=0.5$ does not vary throughout the wave. The electron and proton fluids rotate in phase with a finite wave period in $x$, in which $\phi=\phi_{p}-\phi_{e}=0$ and $u_{x}=u_{x 0}$ throughout the wave. The electron and proton hodographs in Fig. 13 show that the transverse velocities $\left(u_{e y}, u_{e z}\right)$ for the electrons and $\left(u_{p y}, u_{p z}\right)$ for the protons simply rotate in circle with constant radius $u_{e}$ for the electrons (top panel) and radius $u_{p}$ for the protons (lower panel), whilst maintaining a constant phase difference $\phi=\phi_{p}-\phi_{e}=0$ throughout the wave. 

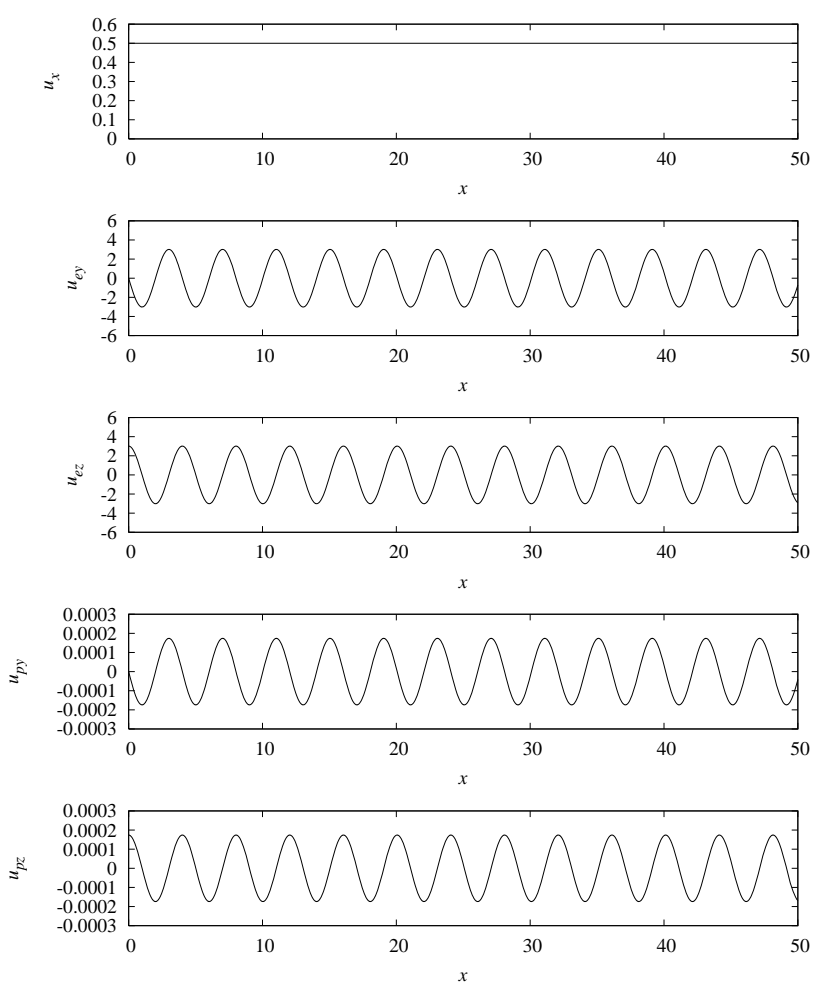

Fig. 12. Velocity components for the case when $R\left(u_{x}\right)$ has a double root. The parameters used were: $u_{x 0}=0.5, M_{\mathrm{Ae} 0}=0.255, \theta=30^{\circ}$ and $u_{e 0}=3.01049$. The fluids simply rotate in phase, maintaining $\phi=0$ and $u_{x}=u_{x 0}$ throughout.

Another double root case for $R\left(u_{x}\right)=0$ occurs if $u_{-}=u_{+}$ (see Proposition 5.6, Eqs. 136-138 et seq.). This occurs when the rotational integral $\mathcal{R}=0$ and the Alfvén Mach number $M_{\mathrm{Ae} 0}$ is given by the equation:

$M_{\mathrm{Ae} 0}^{2} \sec ^{2} \theta=\frac{1}{4}\left(\frac{\mu+1}{\mu}-\frac{\tan ^{2} \theta}{\mu+1}\right)$.

The values

$u_{e 0}=\mu u_{p 0}=\frac{u_{x 0} \sin \theta \cos \theta}{2 M_{\mathrm{Ae} 0}^{2}} \equiv \frac{b}{2}$,

ensure that both the rotational integral $\mathcal{R}=u_{e 0}^{2}-\mu^{2} u_{p 0}^{2}=0$ and the integrability condition $u_{e 0}+\mu u_{p 0}=b$ in Eq. (89) are both satisfied. For this solution,

$u_{+}=u_{-}=u_{x 0}\left(1+\frac{\sin ^{2} \theta}{2(\mu+1) M_{\mathrm{Ae} 0}^{2}}\right) \equiv \frac{H_{1}}{u_{x 0}}$,

are the two equal roots $u_{ \pm}$of $R\left(u_{x}\right)=0$.

Figure 14 shows a plot of $R\left(u_{x}\right)$ versus $u_{x}$ for a case where $u_{-}=u_{+}$is a double root of $R\left(u_{x}\right)$. The parameters are $u_{x 0}=1, \theta=30^{\circ}, \mu=1836$. The parameters $M_{\mathrm{Ae} 0}, u_{e 0}$, $\mu u_{p 0}$, and $u_{ \pm}$are determined from Eqs. (169-171). The

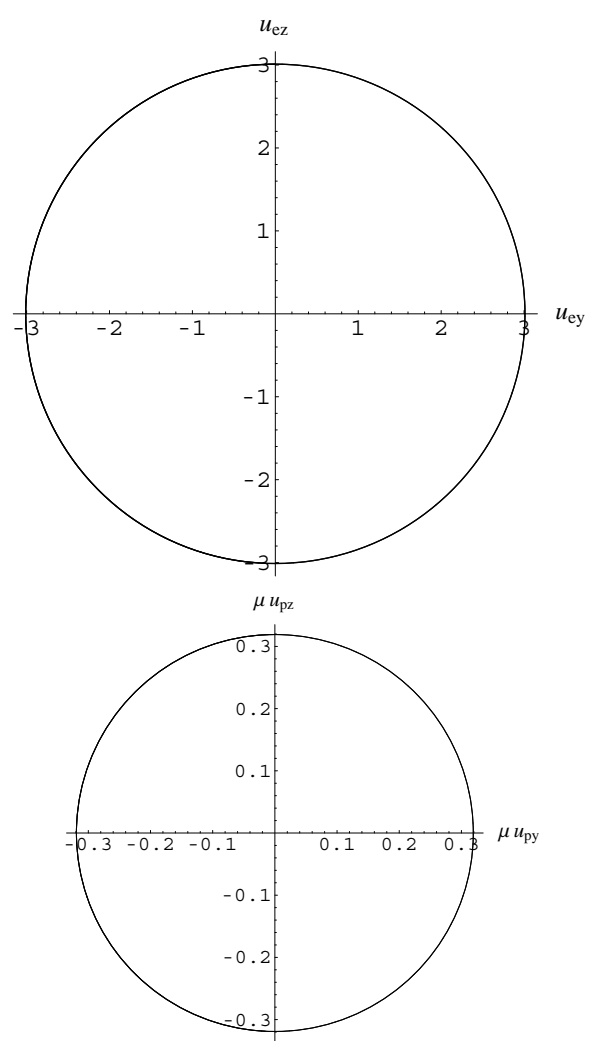

Fig. 13. Perpendicular electron and proton "hodographs" for the case when $R\left(u_{x}\right)$ has a double root. The parameters are the same as in Fig. 12. The fluids simply rotate in phase, maintaining $\phi=0$ and $u_{x}=u_{x 0}$ throughout.

travelling wave solution corresponding to the $R\left(u_{x}\right)$ plot in Fig. 14 has a simple zero $u_{x}=u_{x 0}=1$ and a double zero at $u_{x}=u_{+}=u_{-}=1.00036$. The physical solution is restricted to the range $u_{x 0} \leq u_{x} \leq u_{+}$where $R\left(u_{x}\right) \geq 0$. The point $u_{x}=u_{x 0}$ corresponds to the centre of the wave and the double zero at $u_{x}=u_{+}$corresponds to $x \rightarrow \pm \infty$ where $u_{e} \rightarrow 0$ and $u_{p} \rightarrow 0$. The spatial profiles of the electron and proton transverse fluid velocities and the longitudinal speed $u_{x}$ for the above parameters are displayed in Fig. 15. The wave is an oblique, whistler oscilliton of the type investigated by Sauer et al. (2001, 2002), Dubinin et al. (2003), McKenzie et al. (2004), and Webb et al. (2005). Figure 15 shows only half of the wave in the region $x>0$. The wave is symmetric about $x=0$. Thus, the wave envelope for the transverse velocities, stretching from $x=-\infty$ to $x=+\infty$ has a bell shape profile reminiscent of that of a classical soliton, on which there is superimposed a high wave number oscillating component. The present analysis shows that the obliquely propagating oscilliton is an integrable solution, and that there are very stringent conditions required to obtain an oscilliton in the oblique propagation case. Figure 16 shows hodographs of the transverse electron and proton velocities, which have a distinctive 


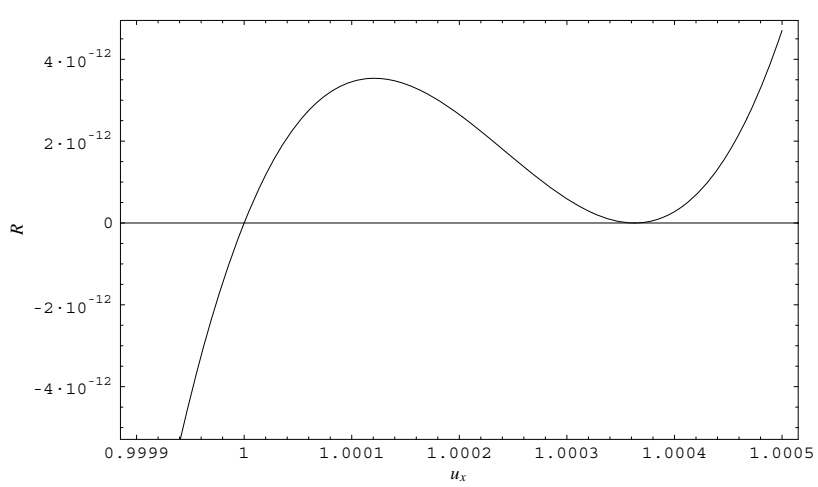

Fig. 14. Plot of $R\left(u_{x}\right)$ for the special case where $\mathcal{R}=0$. The parameters are $u_{x 0}=1, \theta=30^{\circ}, \mu=1836 . M_{\mathrm{Ae} 0}, u_{e 0}$, and $\mu u_{p 0}$ are determined from Eqs. (169-171). This case corresponds to an oscilliton solution.

spiral structure, in which the maximum amplitudes of $u_{e}$ and $\mu u_{p}$ occur at the centre of the wave and with $u_{e} \rightarrow 0$ and $\mu u_{p} \rightarrow 0$ as $x \rightarrow \pm \infty$.

\subsubsection{Rosette winding patterns}

In this section we discuss the results of C. M. Ko (private communication) on the winding pattern of the transverse velocity hodographs of the electron and proton fluids, as illustrated in Figs. 5 and 7. Figure 7 shows clearly that in general, both the electron and proton fluids exhibit rosette patterns in the the $\left(u_{e y}, u_{e z}\right)$ and $\left(u_{p y}, u_{p z}\right)$ hodograph planes. First, it is useful to note that the Hamiltonian contours in the $(\phi, w)$ phase plane in Figs. 1 and 2 split into two different cases depending on whether the contours of $H$ are bounded $\left(\mathcal{C}_{b}\right)$ or unbounded $\left(\mathcal{C}_{u}\right)$ in $\phi$. The bound orbits are restricted to the a region $|\phi(x)| \leq \phi_{m}<\pi$, whereas the unbound orbits assume all values $|\phi(x)| \leq \pi$ (note the contours are $2 \pi$ periodic in $\phi)$. The function $u_{x}(x)$ is a periodic function of $x$ with period $L$, i.e. $u_{x}(x+L)=u_{x}(x)$ where the period $L$ is given by Eq. (158). The conditions for the transverse electron and proton fluid velocity hodographs to consist of a closed pattern of $\mathrm{M}$ large leaves (cloves) are discussed below.

A natural question to ask is: given that the electrons rotate through $N_{e}$ complete orbits in $\phi_{e}$, in which the change of $\phi_{e}, \Delta \phi_{e}=2 \pi N_{e}$ in the $\left(u_{e y}, u_{e z}\right)$ hodograph plane, corresponding to $M$ orbits in the $(\phi, w)$ phase plane, then what are the conditions for the hodograph orbits to consist of closed orbits with $M$ large leaves? Note that one large leaf in the $\left(u_{e y}, u_{e z}\right)$ plane in general corresponds to $\Delta \phi_{e}>2 \pi$ in that plane (i.e. portions of the orbit at small $u_{e}$ can be convoluted and contribute substantially to $\Delta \phi_{e}$ ). A similar question can be posed for the protons. One finds that for bound closed orbits in the $(\phi, w)$ phase plane, the rosette patterns in the $\left(u_{e y}, u_{e z}\right)$ plane with $\Delta \phi_{e}=2 \pi N_{e}$ and in the $\left(u_{p y}, u_{p z}\right)$ plane with $\Delta \phi_{p}=2 \pi N_{p}$ consist of closed orbits with $M$ large
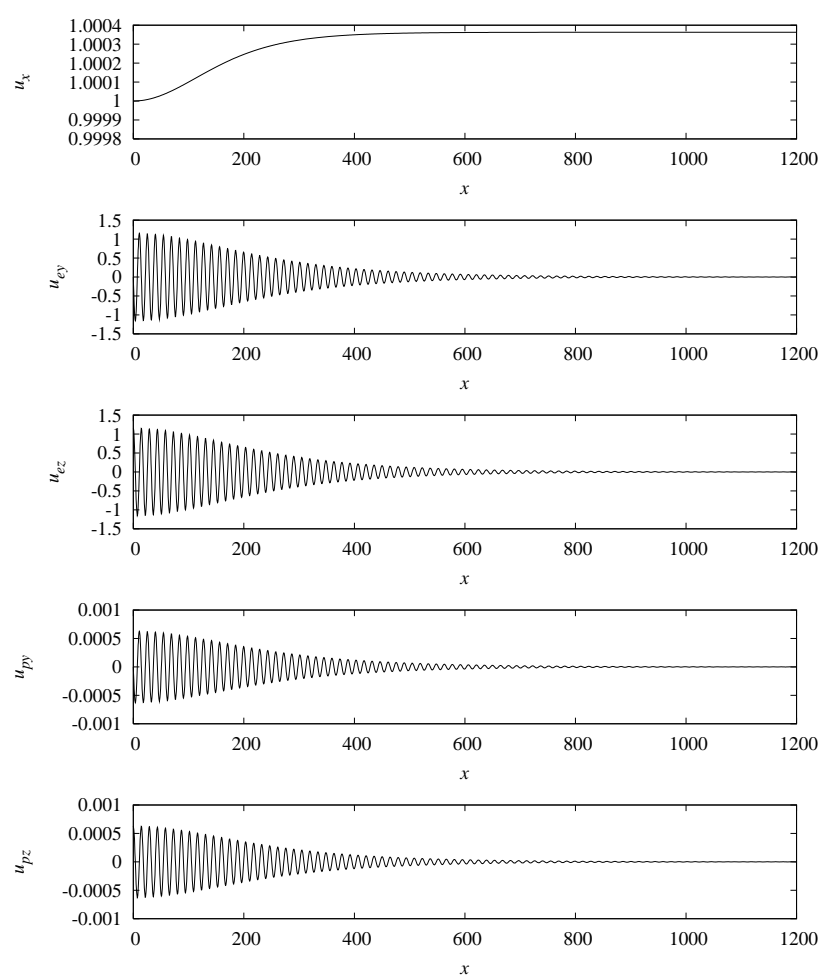

Fig. 15. Velocity components as a function of $x$ for the solution corresponding to the case given in Fig. 14. This is an oscillitontype solution. Only half of the wave is shown. The parameters are $u_{x 0}=1, \theta=30^{\circ}, \mu=1836$, and $M_{\mathrm{Ae} 0}, u_{e 0}$ and $\mu u_{p 0}$ are determined from Eqs. (169-171).

leaves requires that $N_{e}=N_{p}$. However, for the unbound orbits $\left(\mathcal{C}_{u}\right)$ in the $(\phi, w)$ plane, the orbits consist of $M$ large leaves in the hodograph planes only if $N_{p}-N_{e}=M$. The conditions for closed orbits in the hodograph planes also requires $\Delta \tilde{\phi} \equiv[\tilde{\phi}(x+L)-\tilde{\phi}(x)]=4 \pi p / q$ where $p / q$ is a rational number. We refer to $N_{e}$ and $N_{p}$ as the electron and proton winding numbers.

To prove the above assertions, first notice that $d \tilde{\phi} / d x$ is a periodic function of $x$ with period $L$, and hence

$\tilde{\phi}(x)=\left\langle\tilde{\phi}_{x}\right\rangle x+\int_{0}^{x} \delta \tilde{\phi}_{x} d x+\tilde{\phi}(0)$,

gives the variation of $\tilde{\phi}(x)$ with $x$, where $\delta \tilde{\phi}_{x}=\tilde{\phi}_{x}-\left\langle\tilde{\phi}_{x}\right\rangle$ has zero mean over one period $L$ in $x$ and the angle brackets in $\left\langle\tilde{\phi}_{x}\right\rangle$ denote an average over one period in $x$. Setting $x=L$ in Eq. (172) we obtain:

$\left\langle\tilde{\phi}_{x}\right\rangle=\frac{\tilde{\phi}(L)-\tilde{\phi}(0)}{L}=\frac{\Delta \tilde{\phi}}{L}$,

(note $\tilde{\phi}=\phi_{e}+\phi_{p}$ ). Similarly, the variation of $\phi(x)=\phi_{p}-\phi_{e}$ for unbound orbits in the $(\phi, w)$ phase-plane may be written 


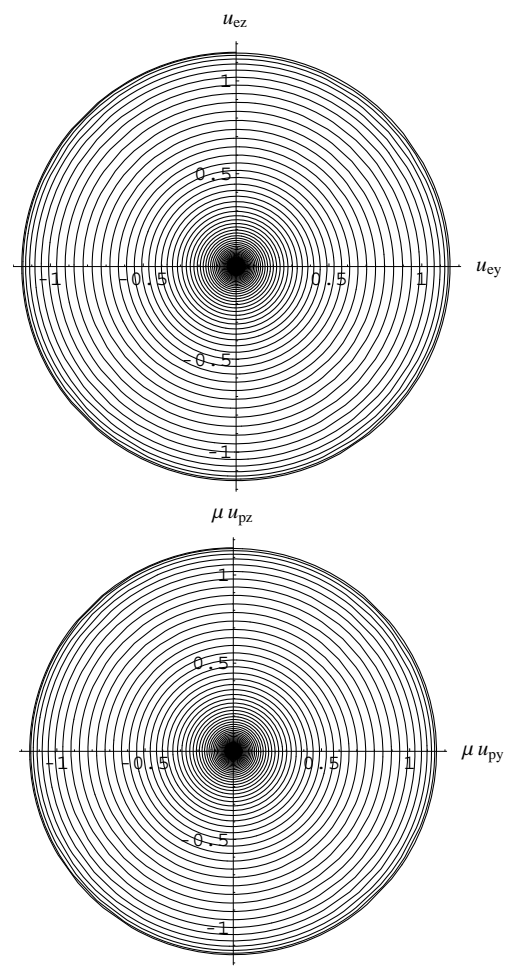

Fig. 16. The perpendicular electron (upper panel) and proton (lower panel) velocity components corresponding to the oscilliton solution of Figs. 14 and 15.

as:

$$
\begin{aligned}
\phi_{u}(x) & =\left\langle\phi_{x}\right\rangle x+\phi(0)+\delta \phi(x) \bmod (2 \pi) \\
& \equiv \frac{2 \pi x}{L}+\delta \phi(x) \bmod (2 \pi),
\end{aligned}
$$

where $\delta \phi=\phi-\langle\phi\rangle$. In our case $\phi(0)=0$ because $\phi_{p 0}=\phi_{e 0}=\pi / 2$, and $\delta \phi(x)=\phi$ where $|\phi| \leq \pi$. Similarly for the bound orbits $\mathcal{C}_{b}$ we obtain:

$\phi_{b}(x)=\delta \phi(x) \bmod (2 \pi)$,

Using Eqs. (172-175) we obtain:

$$
\begin{aligned}
\phi_{p}(x)= & \frac{1}{2}\left(\frac{\left(\Delta \tilde{\phi}+2 \pi \delta_{k u}\right) x}{L}+\tilde{\phi}(0)\right. \\
& \left.+\int_{0}^{x} \delta \tilde{\phi}_{x} d x+\delta \phi(x) \bmod (2 \pi)\right), \\
\phi_{e}(x)= & \frac{1}{2}\left(\frac{\left(\Delta \tilde{\phi}-2 \pi \delta_{k u}\right) x}{L}+\tilde{\phi}(0)\right. \\
& \left.+\int_{0}^{x} \delta \tilde{\phi}_{x} d x-\delta \phi(x) \bmod (2 \pi)\right),
\end{aligned}
$$

where $k=u, b$ refer to unbound and bound orbits in the $(\phi, w)$ plane respectively.
The conditions that the $\phi_{j}(j=e, p)$ undergo a change of $2 \pi N_{j}$ in phase during $M$ periods of $u_{x}(x)$ to give closed orbits of $M$ large leaves in the hodograph planes may be expressed in the form:

$\phi_{j}(x+M L)-\phi_{j}(x)=2 \pi N_{j}, \quad j=e, p$.

Using Eqs. (176-177) for $\phi_{p}$ and $\phi_{e}$, the conditions Eq. (178) reduce to:

$$
\begin{array}{ll}
\frac{N_{p}}{M}=\frac{\Delta \tilde{\phi}}{4 \pi}+\frac{\delta_{k u}}{2}, & (k=u, b), \\
\frac{N_{e}}{M}=\frac{\Delta \tilde{\phi}}{4 \pi}-\frac{\delta_{k u}}{2}, & (k=u, b) .
\end{array}
$$

Thus, if $\Delta \tilde{\phi} /(4 \pi)=p / q$ is a rational number, then the hodograph orbits in the $\left(u_{p y}, u_{p z}\right)$ plane will consist of $M$ large leaves corresponding to a winding number $N_{p}\left(N_{p}\right.$ integer) of the proton orbits. Similarly, for $\Delta \tilde{\phi} /(4 \pi)=p / q$ a rational number, the electron orbits will consist of $M$ leaves in the $\left(u_{e y}, u_{e z}\right)$ plane corresponding to an electron winding number of $N_{e}$. Using Eqs. (179-180) the bound orbits in the $(\phi, w)$ plane, correspond to closed orbits with $M$ large leaves in the transverse hodograph planes if $N_{p}=N_{e}$. Similarly, using Eqs. (179-180), the unbound orbits in the $(\phi, w)$ plane, will correspond to closed orbits of $M$ large leaves in the hodograph planes only if $N_{p}-N_{e}=M$ and $\Delta \tilde{\phi} /(4 \pi)=p / q$ is a rational number. This completes the discussion of the rosette winding patterns of the electron and proton transverse velocities in their respective hodograph planes.

\section{Summary and concluding remarks}

High resolution satellite observations of a variety of wave forms in the near-Earth plasma environment by FAST, Polar, Geotail and Cluster spacecraft (e.g. Cattel et al., 2002, Mozer et al., 1997, Ergun et al., 1998, and Pickett et al., 2003, Dubinin et al., 2007) has stimulated the development of theoretical models to explain the observations based on travelling waves in multi-fluid plasmas (e.g. Sauer et al., 2001, 2002, 2003, Dubinin et al., 2003, McKenzie et al., 2004, Verheest et al., 2004, Webb et al., 2005, 2007, Dubinin et al., 2007).

Analysis of travelling waves in multi-fluid plasmas by Webb et al. $(2005,2007)$ and McKenzie, Mace and Doyle (2007) revealed that many of the travelling wave models are Hamiltonian systems. Webb et al. (2007) showed that the quasi-charge-neutral two-fluid model used in the present paper has a dual Hamiltonian structure.

We used one of the Hamiltonian formulations of Webb et al. (2007), in which the Hamiltonian is identified with the total conserved, longitudinal momentum integral of the system, $P_{x}$, in which the energy integral $\varepsilon=$ const. is regarded as a constraint, and $d / d x$ is the Hamiltonian evolution operator where $x$ denotes position in the travelling wave frame. Hamilton's equations for the system reduce to the transverse 
momentum Eqs. (6) for the electron and proton fluids in the dHT frame, where

$$
\left(q_{1}, p_{1}\right)=\left(u_{e}^{+}, u_{e}^{-}\right) \quad \text { and } \quad\left(q_{2}, p_{2}\right)=\left(u_{p}^{+},-\mu^{2} u_{p}^{-}\right)
$$

are the canonical variables, $u_{e}^{ \pm}=u_{e y} \pm i u_{e z}, u_{p}^{ \pm}=u_{p y} \pm$ $i u_{p z}$ are the complex, transverse electron and proton fluid velocities in the dHT frame, and $\mu=m_{p} / m_{e}$ is the ratio of the proton and electron masses. The transverse electron and proton momentum Eqs. (6) are exactly integrable if the total transverse momentum integrals are zero in the dHT frame (i.e. $\delta_{0}=0$ in Eq. 1). If the initial values for $\left(u_{e y}, u_{e z}\right)$ and $\left(u_{p y}, u_{p z}\right)$ are specified at $x=x_{0}$ in the dHT frame, then $\delta_{0}=0$ if:

$u_{e y 0}+\mu u_{p y 0}=0, \quad u_{e z 0}+\mu u_{p z 0}=\frac{u_{x 0} \sin \theta \cos \theta}{M_{\mathrm{Ae} 0}^{2}} \equiv b$,

where $M_{\mathrm{Ae} 0}=u_{x 0} / V_{A e}$ is the electron Alfvén Mach number of the flow at $x=x_{0}$. If conditions Eq. (182) are satisfied, then Eq. (6) admit an extra integral, namely the rotational integral $\mathcal{R}=u_{e}^{2}-\mu^{2} u_{p}^{2}=$ const., which combined with the other integrals of the system, allows the integration of the system in terms of quadratures over the longitudinal flow speed $u_{x}$. The phase space of the system reduces from a four dimensional space to a two dimensional space in the integrable case (Sect. 4) (this is technically known as Hamiltonian reduction: e.g. Marsden and Ratiu, 1994).

In the integrable cases Eq. (182), the Hamiltonian can be expressed in terms of the canonical variables $(q, p)=(\phi, w)$ where $\phi=\phi_{p}-\phi_{e}$ is the difference between the proton and electron phases for the complex transverse velocities: $u_{j}^{+}=u_{j y}+i u_{j z}=u_{j} \exp \left(i \phi_{j}\right)(j=e, p)$ and $w=u_{e}^{2}$. It turns out, that the conditions Eq. (182) for the system to be integrable force the Hamiltonian $H(\phi, w)$ to take only one value $H=H_{1}$ (see Appendix A). In other words, the system trajectories in the phase space satisfy the equations $H(\phi(x), w(x))=H_{1}$, which essentially fixes the solutions to lie on a particular invariant torus (i.e., only one value of the Hamiltonian contours, namely $H(\phi, w)=H_{1}$ is allowed in the $(\phi, w)$ phase plane: see Eq. (81) et seq.). The integrable solution examples in Sect. 5, were restricted to satisfy a special case of the initial conditions Eq. (182) in which the electron and proton phases have the values:

$\phi_{e 0}=\phi_{p 0}=\pi / 2$ and $u_{e 0}+\mu u_{p 0}=b$,

(more general solutions of Eq. (182) are discussed in Appendix A). Despite this restriction, one obtains a rich variety of travelling wave solutions depending on the initial Alfvén Mach number $M_{\mathrm{Ae} 0}$ and rotational integral $\mathcal{R}=u_{e 0}^{2}-\mu^{2} u_{p 0}^{2}$ of the wave.

The examples of travelling waves for cold plasmas investigated in Sect. 5, depend on the roots of the cubic $R\left(u_{x}\right)$ appearing in the longitudinal structure Eq. (126) for $d u_{x} / d x$.
$R\left(u_{x}\right)=0$ has roots $u=u_{x 0}$ and $u_{x}=u_{ \pm}$, where $u_{ \pm}$are given by Eq. (131). In the double root case $u_{-}=u_{+}$and $u_{x 0}<u_{-}$, one obtains an obliquely propagating whistler oscilliton solution analogous to the parallel propagating whistler oscilliton investigated by Sauer et al. (2001, 2002), Dubinin et al. (2003), McKenzie et al. (2004) and Webb et al. (2005). The hodograph of the transverse fluid velocities in Fig. 16 consist of inward spirals with maximum radius at the centre of the wave at $x=0$ and decrease monotonically to zero as $x \rightarrow \pm \infty$. The double root solution case, in which $u_{x 0}=u_{-}$ and $u_{+}>u_{x 0}$ corresponds to a centre critical point in the $(\phi, w)$ phase plane. For this solution $u_{x}=u_{x 0}$ is constant throughout the wave, and the transverse velocities of the electrons and the protons simply rotate in phase, maintaining $\phi=\phi_{p}-\phi_{e}=0$ throughout the wave. The velocity hodographs in Fig. 13 are circles.

Other solution examples in Sect. 5 correspond to cases where $R\left(u_{x}\right)=0$ has simple roots (i.e. $u_{x 0}, u_{+}$and $u_{-}$are distinct). In these cases, the transverse velocity hodographs show complex rosette type patterns.

The family of travelling wave solutions with rotational integral $\mathcal{R}=0$ is described in detail in Appendix D. This class of solutions is relatively simple to analyse, and the Hamiltonian in this case can be related to the Hamiltonian for parallel propagating whistler waves investigated by Webb et al. (2005).

An analysis of the rosette patterns for the transverse velocities of the electrons $\left(u_{e y}, u_{e z}\right)$ and protons $\left(u_{p y}, u_{p z}\right)$ respectively in Sect. 5.4.1 revealed that for bound orbits in the $(\phi, w)$ Hamiltonian phase plane to correspond to closed orbits with $M$ large leaves required that $N_{e}=N_{p}$ where $N_{e}$ and $N_{p}$ are the winding numbers for the electrons and protons, whereas for unbound orbits in the $(\phi, w)$ phase plane, the condition for the orbits to consist of closed orbits with $M$ large leaves in the hodograph planes requires $N_{p}-N_{e}=M$. The conditions for closed orbits with $M$ large leaves in the hodograph planes also requires $\Delta \tilde{\phi} \equiv[\tilde{\phi}(x+$ $L)-\tilde{\phi}(x)]=4 \pi p / q$ where $p / q$ is a rational number.

The solutions in Figs. 1-16 are a representative class of the integrable cold plasma, travelling wave solutions. However, there are many other solutions, both for the integrable $\left(\delta_{0}=0\right)$ and non-integrable $\left(\delta_{0} \neq 0\right)$ cases that are are not covered by our analysis (e.g., the hot plasma solutions). It remains an open question as to the nature of the non-integrable solutions with $\delta_{0} \neq 0$, and in particular the role of KAM theory in the near-integrable cases (e.g. Zaslavsky et al., 1991), as well as the role of chaos in the non-integrable cases. These problems lie beyond the scope of the present paper, and remain to be explored in other investigations.

\section{Appendix A}

In this appendix we discuss the general solution of the integrability condition $\delta_{0}=0$. The cases for which $\sin \phi_{0}=0$ 
are singular. We first discuss the non-singular case with $\sin \phi_{0} \neq 0$, followed by a discussion of the singular case $\sin \phi_{0}=0$. We show that for $\sin \phi_{0} \neq 0,\left(u_{e 0}, \mu u_{p 0}\right)$ for a fixed $\phi_{0}$ must lie on an ellipse. The condition $\delta_{0}=0$ is equivalent to the two momentum balance Eqs. (14) in the dHT frame. Using

$u_{j y 0}=u_{j 0} \cos \phi_{j 0}, \quad u_{j z 0}=u_{j 0} \sin \phi_{j 0}, \quad j=e, p$,

Equation (14) can be written in the matrix form:

$\mathbf{A}\left(\begin{array}{c}u_{e 0} \\ \mu u_{p 0}\end{array}\right)=\left(\begin{array}{cc}\cos \phi_{e 0} & \cos \phi_{p 0} \\ \sin \phi_{e 0} & \sin \phi_{p 0}\end{array}\right)\left(\begin{array}{c}u_{e 0} \\ \mu u_{p 0}\end{array}\right)=\left(\begin{array}{l}0 \\ b\end{array}\right)$,

The determinant of the matrix $\mathbf{A}, \operatorname{det} \mathbf{A}=\sin \phi_{0}$, in Eq. (A2). Assuming that $\sin \phi_{0} \neq 0$, Eq. (A2) has the general solution:

$\left(\begin{array}{c}u_{e 0} \\ \mu u_{p 0}\end{array}\right)=\frac{b}{\sin \phi_{0}}\left(\begin{array}{c}-\cos \phi_{p 0} \\ \cos \phi_{e 0}\end{array}\right)$.

Note that we require $u_{e 0}$ and $u_{p 0}$ must both be non-negative.

Writing $\left(u_{e 0}, \mu u_{p 0}\right)=(\xi, \eta)$, Eq. (A3) can be combined to give the equations:

$\xi+\eta \cos \phi_{0}=b \sin \phi_{e 0}$,

$\eta \sin \phi_{0}=b \cos \phi_{e 0}$.

Squaring and adding the two equations in Eq. (A4) gives the equation:

$\xi^{2}+\eta^{2}+2 \xi \eta \cos \phi_{0}=b^{2}$.

Equation (A5) is that of an ellipse. To show this, introduce new coordinates $\left(\xi^{\prime}, \eta^{\prime}\right)$ corresponding to a rotation of coordinates through an angle $\Omega$ :

$$
\left(\begin{array}{l}
\xi \\
\eta
\end{array}\right)=\left(\begin{array}{cc}
\cos \Omega & -\sin \Omega \\
\sin \Omega & \cos \Omega
\end{array}\right)\left(\begin{array}{l}
\xi^{\prime} \\
\eta^{\prime}
\end{array}\right),
$$

and choosing $\Omega=\pi / 4$ corresponding to a rotation of $45^{\circ}$, Eq. (A5) reduces to the standard equation for an ellipse:

$$
\frac{\xi^{\prime 2}}{\alpha^{2}}+\frac{\eta^{\prime 2}}{\beta^{2}}=1
$$

where

$$
\alpha=\frac{b}{\sqrt{2}\left|\cos \left(\phi_{0} / 2\right)\right|} \quad \text { and } \quad \beta=\frac{b}{\sqrt{2}\left|\sin \left(\phi_{0} / 2\right)\right|} .
$$

If $\phi_{0}<\pi / 2$, then $\beta$ and $\alpha$ correspond to the semi-major and semi-minor axes of the ellipse respectively. The semi-minor axis $\xi^{\prime}$ lies along the line $\eta=\xi$ and the semi-major axis $\eta^{\prime}$ lies along the line $\eta=-\xi$ corresponding to a $45^{\circ}$ rotation of the $\xi$ and $\eta$ axes. If $\pi / 2<\phi_{0}<\pi$ then $\xi^{\prime}$ is the major axis and $\eta^{\prime}$ is the minor axis. Again note that $\xi=u_{e 0}$ and $\eta=\mu u_{p 0}$ must be non-negative for a physical solution.
In the limit as $\phi_{0} \rightarrow 0$ Eq. (A5) reduces to

$u_{e 0}+\mu u_{p 0}=b$.

The solution Eq. (A9) corresponds to the initial data

$\phi_{0}=0, \quad \phi_{p 0}=\phi_{e 0}=\frac{\pi}{2}$,

of Eq. (81), investigated in detail in Sect. 5. If $0<u_{e 0}<b / 2$ then the rotational integral $\mathcal{R}<0$, but if $b / 2<u_{e 0}<b, \mathcal{R}>0$ and $\mathcal{R}=0$ if $u_{e 0}=b / 2$.

The solution case

$\phi_{0}=\pi, \quad \phi_{p 0}=\frac{\pi}{2}, \quad \phi_{e 0}=-\frac{\pi}{2}, \quad \mu u_{p 0}=u_{e 0}+b$,

and the case

$\phi_{0}=-\pi, \quad \phi_{p 0}=-\frac{\pi}{2}, \quad \phi_{e 0}=\frac{\pi}{2}, \quad u_{e 0}=\mu u_{p 0}+b$,

can also be thought of as degenerate ellipses in the limit as $\phi_{0} \rightarrow \pm \pi$. For Eq. (A11) $\mathcal{R}<0$ and for Eq. (A12) $\mathcal{R}>0$. In the case Eq. (A9), the semi-major axis $\beta=b /\left|\sqrt{2} \sin \left(\phi_{0} / 2\right)\right| \rightarrow \infty$ as $\phi_{0} \rightarrow 0$. Similarly in cases Eq. (A11) and Eq. (A12) the semi-major axis $\beta=$ $b /\left(\sqrt{2}\left|\cos \left(\phi_{0} / 2\right)\right|\right) \rightarrow \infty$ as $\phi_{0} \rightarrow \pm \pi$.

The value of the Hamiltonian $H=H_{1}$ at the initial point $x=x_{0}$ from Eq. (81) is given by:

$H_{1}=u_{x 0}^{2}+\frac{M_{A 0}^{2}}{2(\mu+1)^{2}}\left(u_{e 0}^{2}+\mu^{2} u_{p 0}^{2}+2 \mu u_{p 0} u_{e 0} \cos \phi_{0}\right)$.

Using Eq. (A5) with $\xi=u_{e 0}$ and $\eta=\mu u_{p 0}$, Eq. (A13) reduces to:

$H_{1}=u_{x 0}^{2}+\frac{M_{A 0}^{2} b^{2}}{2(\mu+1)^{2}}$.

Using the expression Eq. (13) for $b$ in Eq. (A14) we obtain:

$H_{1}=u_{x 0}^{2}\left(1+\frac{\tan ^{2} \theta}{2 M_{A 0}^{2}}\right) \equiv u_{x 0}^{2}\left(1+\frac{\sin ^{2} \theta}{2(\mu+1) M_{\mathrm{Ae} 0}^{2}}\right)$,

which is the result Eq. (87) for $H_{1}$.

\section{Appendix B}

In this appendix we discuss the critical points of the Hamiltonian $H_{0}=2(\mu+1) \cos \theta H$ for the travelling wave solutions. We show that the point $\left(\phi_{1}, w_{1}\right)=\left(0, w_{c}\right)$ on the line $\phi=0$ in the $(\phi, w)$ plane where $d \phi / d x=0$ is a centre critical 
point. At this point $d \phi / d x=f\left(u_{x}\right)=0$ and $d w / d x=0$. The points $\left(\phi_{2}, w_{2}\right)=(-\pi / 2,0)$ and $\left(\phi_{3}, w_{3}\right)=(\pi / 2,0)$ could be thought of as critical points in the sense that $H_{0 w} \rightarrow 0$ and $H_{0 \phi} \rightarrow 0$ along the ray direction $\delta \phi / \delta u_{e}=\alpha_{2}$ in Eq. (109). However, along the ray $\delta \phi=0, d \phi / d x=H_{0 w}$ becomes unbounded as one approaches the critical point. In other words, the points $( \pm \pi / 2,0)$ are non-standard critical points.

Below we study Hamilton's equations in the vicinity of these points. Linearizing Hamilton's Eqs. (61) and (62) about the centre critical point $\left(\phi_{1}, w_{1}\right)$ we obtain the matrix equation system:

$$
\frac{d}{d x}\left(\begin{array}{c}
\delta \phi \\
\delta w
\end{array}\right)=\mathbf{A}\left(\begin{array}{c}
\delta \phi \\
\delta w
\end{array}\right), \quad \mathbf{A}=\left(\begin{array}{cc}
H_{0, w \phi} & H_{0, w w} \\
-H_{0, \phi \phi} & -H_{0, \phi w}
\end{array}\right)
$$

Searching for linearized solutions of the linearized Hamilton's equations $(\mathrm{C} 1)$ of the form:

$\left(\begin{array}{l}\delta \phi \\ \delta w\end{array}\right)=\mathbf{r} \exp (\lambda x)$

results in the eigen-equations:

$(\mathbf{A}-\lambda \mathbf{I}) \mathbf{r}=0 \quad$ where $\quad \operatorname{det}(\mathbf{A}-\lambda \mathbf{I})=0$.

Evaluation of the determinantal equation $\operatorname{det}(\mathbf{A}-\lambda \mathbf{I})=0$ in Eq. (B3) gives the eigenvalues $\lambda$ in the form:

$\lambda^{2}=H_{0, w \phi}^{2}-H_{0, \phi \phi} H_{0, w w} \equiv G=-\operatorname{det}(\mathbf{A})$.

The nature of the critical point depends on whether $G>0$ or $G<0$. For $G>0$ the critical point is a saddle, but if $G<0$ the point is a centre.

Using the Hamiltonian Eq. (63) for the cold gas case with $M_{A}=1$, we find:

$$
\begin{aligned}
H_{0, \phi \phi}= & -\frac{2 M_{A}^{2} u_{x 0} \cos \theta}{\mu+1} \mu u_{p} u_{e} \cos \phi, \\
H_{0, \phi w}= & -\frac{M_{A}^{2} u_{x 0} \cos \theta}{\mu+1} \frac{\left(u_{e}^{2}+\mu^{2} u_{p}^{2}\right) \sin \phi}{\mu u_{p} u_{e}} \\
H_{0, w w}= & -\frac{M_{A}^{2} u_{x 0} \cos \theta}{\mu+1} \frac{\left(u_{e}^{2}-\mu^{2} u_{p}^{2}\right)^{2} \cos \phi}{2 \mu^{3} u_{p}^{3} u_{e}^{3}} \\
& -\frac{(\mu+1) \cos \theta}{2 \mu^{2} u_{x}^{3}} .
\end{aligned}
$$

Using Eq. (B5) in Eq. (B4), we find that at the critical point $\left(\phi_{1}, w_{1}\right)=\left(0, w_{1}\right)$ that

$$
\lambda^{2}=-\frac{M_{A}^{4} u_{x 0}^{2} \cos ^{2} \theta \mu u_{p} u_{e}}{(\mu+1)^{2}}\left(\frac{\left(u_{e}^{2}-\mu^{2} u_{p}^{2}\right)^{2}}{\mu^{3} u_{p}^{3} u_{e}^{3}}+\frac{(\mu+1)^{2}}{\mu u_{x}^{3}}\right) .
$$

Because $\lambda^{2}<0$ this implies $\left(\phi_{1}, w_{1}\right)$ is a centre. Note that $\lambda= \pm i|\lambda|$ is pure imaginary in Eq. (B6). In the neighborhood of $\left(\phi_{2}, w_{2}\right)$ and $\left(\phi_{3}, w_{3}\right)$, one can show that $G>0$, indicating saddle type behaviour. However $G \rightarrow \infty$ in the neighborhood of the points $( \pm \pi / 2,0)$, so that the usual procedure of linearizing about these points becomes questionable.

\section{Appendix C}

In this appendix we discuss the conditions for the Hamiltonian contour $H=H_{1}$ to correspond to a separatrix in the $(\phi, w)$ phase-plane where $w=u_{e}^{2}$. There are two cases: either (i) the contour passes through the points $\left(\phi, u_{e}\right)=( \pm \pi / 2,0)$ or (ii) the contour passes through the points where $\phi= \pm \pi / 2$ and $u_{p}=0$. In Case (i) the rotational integral $\mathcal{R}=u_{e}^{2}-$ $\mu^{2} u_{p}^{2}<0$ but in Case (ii) $\mathcal{R}>0$.

\section{Proposition C1}

Consider the integrability constraint Eq. (89) for which $\delta_{0}=0$, i.e.

$\phi_{e 0}=\phi_{p 0}=\frac{\pi}{2}, \quad \phi_{0}=0, \quad u_{e 0}+\mu u_{p 0}=b$.

A condition for the contour $H=H_{1}$ of the Hamiltonian Eq. (75) for the cold plasma case to be a separatrix passing through the points $(\phi, w)=( \pm \pi / 2,0)$ in the $(\phi, w)$ phase plane, where $w=u_{e}^{2}$, is that either

$u_{e 0}=0$,

or

$u_{e 0}=\frac{2(\mu+1) u_{x 0} \tan \theta}{(\mu+1)^{2} / \mu-\tan ^{2} \theta}$.

In the case Eq. (C3)

$$
\begin{aligned}
\mu u_{p 0} & =b-u_{e 0} \\
& \equiv \frac{u_{x 0} \sin \theta \cos \theta}{M_{\mathrm{Ae} 0}^{2}}\left(1-\frac{2(\mu+1) M_{\mathrm{Ae} 0}^{2} \sec ^{2} \theta}{(\mu+1)^{2} / \mu-\tan ^{2} \theta}\right) .
\end{aligned}
$$

In order that $u_{e 0}$ and $\mu u_{p 0}$ in Eqs. (C3) and (C4) are nonnegative requires

$$
\begin{aligned}
& \tan ^{2} \theta<\left((\mu+1)^{2} / \mu,\right. \\
& M_{\mathrm{Ae} 0}^{2} \sec ^{2} \theta<\frac{\mu+1}{2 \mu}\left(1-\frac{\mu \tan ^{2} \theta}{(\mu+1)^{2}}\right) .
\end{aligned}
$$

The rotational integral $\mathcal{R}<0$ if the separatrix passes through $(\phi, w)=( \pm \pi / 2,0)$.

From Eq. (94) $u_{e}=0$ when

$u_{x}^{2}=u_{x 1}^{2}=u_{x 0}^{2}+u_{e 0}^{2} / \mu$.

From Eq. (C1) the rotational integral

$$
\mathcal{R}=u_{e 0}^{2}-\mu^{2} u_{p 0}^{2}=b\left(b-2 \mu u_{p 0}\right)=-\mu^{2} u_{p s}^{2},
$$

where $u_{p}=u_{p s}$ at $u_{x}=u_{x 1}$ and $u_{e}=0$. Using Eq. (75) the value of $H$ passing through the initial point $x=x_{0}$ where $u_{e}=u_{e 0}, u_{p}=u_{p 0}$ and $\phi=\phi_{0}$ for the cold plasma case is:

$$
H=H_{1}=u_{x 0}^{2}+\frac{M_{A}^{2} u_{x 0}^{2} b^{2}}{2(\mu+1)^{2}} .
$$


If the $H=H_{1}$ contour corresponds to a separatrix, then

$$
H=H_{s}=u_{x 0}\left(u_{x 0}^{2}+\frac{u_{e 0}^{2}}{\mu}\right)^{1 / 2}+\frac{M_{A}^{2} u_{x 0}^{2}}{2(\mu+1)^{2}} b\left(b-2 u_{e 0}\right) .
$$

Equating the expressions for $H$ in Eqs. (C9) and (C10) and noting that

$M_{A}^{2} b=\frac{(\mu+1) \tan \theta}{u_{x 0}}$,

gives the equation:

$u_{e 0}\left[\left(\frac{(\mu+1)^{2}}{\mu}-\tan ^{2} \theta\right) u_{e 0}-2(\mu+1) u_{x 0} \tan \theta\right]=0$.

Equation (C12) implies that $u_{e 0}$ is given by either (C2) or (C3). This completes the proof.

\section{Proposition C2}

If the integrability constraint Eq. (C1) is enforced, the contour $H=H_{1}$ of the Hamiltonian Eq. (75) for a cold plasma, will be a separatrix passing through the points $\phi= \pm \pi / 2$ and $u_{p}=0$, if either:

$u_{p 0}=0$

or

$\mu u_{p 0}=\frac{2(\mu+1) u_{x 0} \tan \theta}{(\mu+1)^{2} / \mu-\tan ^{2} \theta}$.

The value of $u_{e 0}$ is $u_{e 0}=b$ in case Eq. (C13). In case Eq. (C14):

$$
\begin{aligned}
u_{e 0} & =b-\mu u_{p 0} \\
& \equiv \frac{u_{x 0} \sin \theta \cos \theta}{M_{\mathrm{Ae} 0}^{2}}\left(1-\frac{2(\mu+1) M_{\mathrm{Ae} 0}^{2} \sec ^{2} \theta}{(\mu+1)^{2} / \mu-\tan ^{2} \theta}\right) .
\end{aligned}
$$

Proof: The method of proof is essentially the same as in Proposition $\mathrm{C} 1$, but the details are different.

\section{Appendix D}

In this appendix we consider the class of travelling waves in which the rotational integral $\mathcal{R}=0$. This is instructive on two accounts. First, the case $\mathcal{R}=0$ can be related to the Hamiltonian formulation for parallel travelling whistler waves investigated by Webb et al. (2005). Secondly, the formulae for the roots of the cubic equation $R\left(u_{x}\right)=0$ occurring in the longitudinal structure Eq. (126), namely $u_{x}=u_{x 0}$ and $u_{x}=u_{ \pm}$are relatively simple in the case $\mathcal{R}=0$. This allows one to obtain a picture of the relative movement of the roots of $R\left(u_{x}\right)$, $u_{x}=u_{x 0}, u_{x}=u_{ \pm}$with increasing electron Alfvén Mach number $M=M_{\mathrm{Ae} 0} \sec \theta$, and hence to classify the different travelling wave types that occur for the case $\mathcal{R}=0$.

Setting the rotational integral $\mathcal{R}=u_{e 0}^{2}-\mu^{2} u_{p 0}^{2}=0$, in Eqs. (84-85), the formulae for $u_{p}(w)$ and $u_{x}(w)$ become:

$\mu u_{p}(w)=w^{1 / 2}, \quad u_{x}(w)=\left(u_{x 0}^{2}+\left(u_{e 0}^{2}-w\right) / \mu\right)^{1 / 2}$,

and the Hamiltonian Eq. (84) reduces to:

$$
H=u_{x 0}\left(u_{x 0}^{2}+\frac{u_{e 0}^{2}-w}{\mu}\right)^{1 / 2}+\frac{M_{\mathrm{Ae} 0}^{2} \sec ^{2} \theta}{\mu+1} w(1+\cos \phi) .
$$

Setting $u_{x 0}=1$ and $u_{e 0}=0$ in Eq. (D2) we obtain the Hamiltonian

$H=\left(1-\frac{w}{\mu}\right)^{1 / 2}+\frac{M_{\mathrm{Ae} 0}^{2} \sec ^{2} \theta}{\mu+1} w(1+\cos \phi)$,

which is the Hamiltonian used by Webb et al. (2005) (except for a scaling factor of $2 \mu$ ) in describing parallel propagating whistler waves.

Next consider the roots of the cubic polynomial $R\left(u_{x}\right)$ given in Eq. (130) and in the longitudinal structure Eq. (126), in the special case where $\mathcal{R}=0$. Note that $\mathcal{R}=0$ if $u_{e 0}=\mu u_{p 0}$, and that for this case $\Delta$ in Eq. (131) is a perfect square. In this case we obtain:

$u_{ \pm}=\frac{u_{*}^{2} \pm\left|u_{*}^{2}-H_{1}\right|}{u_{x 0}}$,

where

$$
\begin{aligned}
& u_{*}^{2}=\frac{u_{x 0}^{2}}{4 M_{\mathrm{Ae} 0}^{2}(\mu+1)}\left(\sin ^{2} \theta+\frac{(\mu+1)^{2}}{\mu} \cos ^{2} \theta\right) . \\
& H_{1}=u_{x 0}^{2}\left(1+\frac{\sin ^{2} \theta}{2 M_{\mathrm{Ae} 0}^{2}(\mu+1)}\right) .
\end{aligned}
$$

Here $H_{1}$ is the value of the Hamiltonian integral Eq. (87) and $u_{*}^{2}$ is defined in Eq. (139). There are two cases to consider, listed below.

Case

(i) $u_{*}^{2} \geq H_{1}$

In this case, we obtain from Eq. (D4) the expressions:

$$
\begin{aligned}
& u_{+}=\frac{u_{x 0}}{M_{\mathrm{Ae} 0}^{2} \sec ^{2} \theta}\left(\frac{\mu+1}{2 \mu}-M_{\mathrm{Ae} 0}^{2} \sec ^{2} \theta\right), \\
& u_{-}=u_{x 0}\left(1+\frac{\sin ^{2} \theta}{2 M_{\mathrm{Ae} 0}^{2}(\mu+1)}\right) \equiv \frac{H_{1}}{u_{x 0}},
\end{aligned}
$$

for the roots $u_{ \pm}$. The condition $u_{*}^{2} \geq H_{1}$ is equivalent to

$M_{\text {Ae } 0}^{2} \sec ^{2} \theta \leq \frac{1}{4}\left(\frac{\mu+1}{\mu}-\frac{\tan ^{2} \theta}{\mu+1}\right)$. 
The roots of $R\left(u_{x}\right)=0$ are ordered so that $u_{x 0}<u_{-}<u_{+}$. Case (ii) $u_{*}^{2} \leq H_{1}$

From Eq. (D4) we obtain:

$$
\begin{aligned}
& u_{+}=u_{x 0}\left(1+\frac{\sin ^{2} \theta}{2 M_{\mathrm{Ae} 0}^{2}(\mu+1)}\right) \equiv \frac{H_{1}}{u_{x 0}}, \\
& u_{-}=\frac{u_{x 0}}{M_{\mathrm{Ae} 0}^{2} \sec ^{2} \theta}\left(\frac{\mu+1}{2 \mu}-M_{\mathrm{Ae} 0}^{2} \sec ^{2} \theta\right),
\end{aligned}
$$

The condition $u_{*}^{2} \leq H_{1}$ is equivalent to

$M_{\mathrm{Ae} 0}^{2} \sec ^{2} \theta \geq \frac{1}{4}\left(\frac{\mu+1}{\mu}-\frac{\tan ^{2} \theta}{\mu+1}\right)$.

We require:

$\frac{1}{4}\left(\frac{\mu+1}{\mu}-\frac{\tan ^{2} \theta}{\mu+1}\right) \leq M_{\mathrm{Ae} 0}^{2} \sec ^{2} \theta \leq \frac{\mu+1}{2 \mu}$,

to ensure that $u_{-} \geq 0$. Here $u_{-}=0$ at the upper limit on $M_{\mathrm{Ae} 0}^{2} \sec ^{2} \theta$ in Eq. (D13).

Based on the formulae Eqs. (D7-D13) we obtain the following classification for the roots of $R\left(u_{x}\right)=0$. Using the notation

$$
\begin{aligned}
& M^{2}=M_{\mathrm{Ae} 0}^{2} \sec ^{2} \theta, \quad M_{1}^{2}=\frac{1}{4}\left(\frac{\mu+1}{\mu}-\frac{\tan ^{2} \theta}{\mu+1}\right), \\
& M_{2}^{2}=\frac{\mu+1}{4 \mu}, \quad M_{3}^{2}=\frac{\mu+1}{2 \mu},
\end{aligned}
$$

we find for increasing $M$ the following scheme:

(i). If $M^{2}<M_{1}^{2}$ then $u_{x 0}<u_{-}<u_{+}$.

(ii). If $M^{2}=M_{1}^{2}$, then $u_{-}=u_{+}>u_{x 0}$, and $u_{-}=u_{+}$is a double root. This case corresponds to an oscilliton solution.

(iii). If $M_{1}^{2}<M^{2}<M_{2}^{2}$, then $u_{x 0}<u_{-}<u_{+}$is the order of the roots of $R\left(u_{x}\right)$. This is the same order for the roots in (i).

(iv). If $M^{2}=M_{2}^{2}$, then $u_{-}=u_{x 0}<u_{+}$. This is a double root case. $u_{x}=u_{x 0}$ is a centre critical point and $u_{x}=u_{x 0}$ is constant throughout the wave. Rotation of the transverse fluid velocity components $u_{j y}$ and $u_{j z}$ of the electron and proton fluids $(j=e, p)$ as well as rotation of the transverse magnetic field occurs.

(v). If $M_{2}^{2}<M^{2}<M_{3}^{2}$ then $u_{-}<u_{x 0}<u_{+}$. Note that the order of the roots $u_{-}$and $u_{x 0}$ is reversed compared to (i).

(vi). If $M^{2}=M_{3}^{2}$, then $u_{-}=0$. The order of the roots is the same as in (v). For $M^{2}>M_{3}^{2}, u_{-}<0$. Solutions exist for $u_{-}<0$, but they must involve a shock, because $u_{x}>0$ is required for physical solutions.

Using the expression Eq. (123) for $D\left(u_{e 0}\right)$ in terms of $\mathcal{R}$ and $\delta_{1}$, with $\mathcal{R}=0$, we obtain:

$D=-\frac{b^{2} \delta_{1}}{4}$ where $\delta_{1}$ is given by Eq. (118). We find that $D<0$ if $M<M_{2}$ (i.e. $\left.\delta_{1}>0\right)$ and $D>0$ if $M>M_{2}$. If $D<0$ then $u_{x 0}<u_{-}<u_{+}$, and if $D>0$, then $u_{-}<u_{x 0}<u_{+}$as discussed in Eq. (142) et seq.

Acknowledgements. The work of GMW and GPZ was supported in part by NASA grant NN05GG83G and NSF grant nos. ATM-03-17509 and ATM-04-28880.The work of C. M. Ko was carried out for the most part, during sabbatical leave at the IGPP at the University of California Riverside in 2006 and 2007. C. M. Ko was supported by the Taiwan National science council grant NSC95-2112-M-008-006. C. M. Ko and J. F. McKenzie acknowledge support from the IGPP at the University of California Riverside, and the hospitality of the director of the IGPP, G. P. Z.

Edited by: R. Grimshaw

Reviewed by: G. Belmont and another anonymous referee

\section{References}

Abramowitz, M. and Stegun, I. A.: Handbook of Mathematical Functions, (Dover: New York), 589 pp., 1965.

Bridges, T. J.: Spatial Hamiltonian structure, energy flux and the water wave problem, Proc. Roy. Soc. Lond. A, 439, 297-315, 1992.

Bridges, T. J.: Multi-symplectic structures and wave propagation, Math. Proc. Camb. Philos. Soc., 121, 147-190, 1997a.

Bridges, T. J.: A geometric formulation of the conservation of wave action and its implications for signature and the classification of instabilities, Proc. Roy. Soc. A, 453, 1365-1395, 1997b.

Cattel, C., Johnson, L., Bergman, R., Klumpar, D., Carlson, C., McFadden, J., Strangeway, R., Ergun, R., Sigsbee, K., and Pfaff, R.: FAST observations of discrete electrostatic waves in association with down-going ion beams in the auroral zone, J. Geophys. Res., 107, A9, 1238, doi:0.1.029/2001JA000254, 2002.

Chen, F. F.: Introduction to plasma physics and controlled fusion, (New York: Plenum Press), 136 pp., 1984.

Cotter, C. J., Holm, D. D. and Hydon, P. E.: Multi-symplectic formulation of fluid dynamics using the inverse map, Proc. Roy. Soc. Lond. A, 463, 2617-2687, 2007.

de Hoffmann, F. and Teller, E.: Magnetohydrodynamic shocks, Phys. Rev., 80, 4, 692-703, 1950.

Dubinin, E., Sauer, K. and McKenzie, J. F.: Nonlinear stationary whistler waves and whistler solitons (oscillitons). Exact solutions, J. Plasma Phys., 69, 305-330, 2003.

Dubinin, E. M., Maksimovic, M., Cornilleau-Werhlin, N., Fontaine, D., Travnicek, P., Mangeney, A., Alexandovna, O., Sauer, K., Fraenz, M., Dandouras, I., Lucek, E., Fazakerley, A., Balogh, A., and Andre, M.: Coherent whistler emissions in the magnetosphere-Cluster observations, Ann. Geophys., 25, $303-$ 315, 2007, http://www.ann-geophys.net/25/303/2007/.

Drury, L. O.'C.: An introduction to diffusive shock acceleration of energetic charged particles in tenuous plasmas, Rept. Progress Phys., 46, 973-1027, 1983.

Elsässer, K.: Lagrangian stability of fluids and multifluid plasmas, Phys. Plasmas, 1, 3161-3173, 1994.

Ergun, R., Carlson, C. W., McFadden, J. P., Mozer, F. S., Delory, G. T., Peria, W., Chaston, C. C., Temerin, M., Roth, I., Muschetti, L., Elphic, R., Strangeway, R., Pfaff, R., Cattel, C. A., Klumpar, 
D., Shelley, E., Peterson, W., Möbius, E., and Kistler, L.: FAST satellite observations of large-amplitude solitary structures, Geophys. Res. Lett., 25, 12, 2041-2044, 1998.

Holm, D. D. and Kupershmidt, B. A.: Poisson brackets and Clebsch representations for magnetohydrodynamics, multi-fluid plasmas and elasticity, Physica D, 6, 347-363, 1983.

Hydon, P. E.: Multi-symplectic conservation laws for differential and differential-difference equations, Proc. Roy. Soc. A, 461, 1627-1637, 2005.

Mace, R. L., McKenzie, J. F., and Webb, G. M.: Conservation laws for steady flow and solitons in a multi-fluid plasma re-visited, Phys. Plasmas, 14, 012310, doi:10.1063/1.2423250, 2007.

Magri, F.: A simple model of the integrable Hamiltonian equation, J. Math. Phys., 19, 5, 1156-1162, 1978.

Marsden, J. E. and Ratiu, T. S.: Introduction to Mechanics and Symmetry, New York: Springer, pp. II, 308, 1994.

Matsumoto, H., Kojima, H., Miyatake, T., et al.: Electrostatic solitary waves (ESW) in the magnetotail: BEN wave forms observed by Geotail, Geophys. Res. Lett., 21, 2915-2918, 1994.

McKenzie, J. F. and Doyle, T. B.: Oblique solitons in a cold magnetized plasma, Phys. Plasmas, 8, 4367-4374, 2001.

McKenzie, J. F. and Doyle, T. B.: A unified view of acousticelectrostatic solitons in complex plasmas, New J. Phys., 5, 26.126.10, 2003.

McKenzie, J. F., Dubinin, E., Sauer, K., and Doyle, T. B.: The application of the constants of the motion to nonlinear stationary waves in complex plasmas: a unified fluid dynamic viewpoint, $\mathbf{J}$. Plasma Phys., 70, 431-462, 2004.

McKenzie, J. F., Dubinin, E. M., and Sauer, K.: Relativistic whistler oscillitons-do they exist?, Nonl. Processes Geophys., 12, 425431, 2005.

McKenzie, J. F., Mace, R. L., and Doyle, T. B.: Nonlinear Hall MHD and electrostatic ion-cyclotron waves: a Hamiltoniangeometric viewpoint, J. Plasma Phys., 73, 5, 687-700, 2007.

Mozer, F. S., Ergun, R., Temerin, M., Cattel, C., Dombeck, J., and Wygant, J.: New features in time domain electric field structures in the auroral acceleration region, Phys. Rev. Lett., 79, 12811284, 1997.

Olver, P. J.: Applications of Lie Groups to Differential Equations, 2nd Edition, New York: Springer, 412 pp., 1993.

Pickett, J. S., Menietti, J. D., Gurnett, D. A., Tsurutani, B., Kintner, P. M., Klatt, E., and Balogh, A.: Solitary potential structures observed in the magnetosheath by the Cluster spacecraft, Nonlin. Processes. Geophys., 10, 3-11, 2003.

Pottelette, R., Treuman, R., and Berthomier, M.: Auroral plasma turbulence and the cause of AKR fine structure, J. Geophys. Res., 106, 8465, doi:10.1029/200JA000098, 8645-8476, 2001.
Ramani, A., Dorizzi, B., and Grammaticos, G.: Painlevé conjecture revisited, Phys. Rev. Lett., 49, 1539-1541, 1982.

Sahraoui, F., Belmont, G., and Rezeau, L.: Hamiltonian canonical formulation of Hall-magnetohydrodynamics: toward an application to weak turbulence theory, Phys. Plasmas, 10, 5, 1325-1337, 2003.

Sauer, K., Dubinin, E., and McKenzie, J. F.: New type of soliton in bi-ion plasmas and possible implications, Geophys. Res. Lett., 28, 3589-3592, 2001.

Sauer, K., Dubinin, E., and McKenzie, J. F.: Coherent wave emission by whistler oscillitons: application to lion roars, Geophys. Res. Lett., 29, 2226-2229, 2002.

Sauer, K., Dubinin, E., and McKenzie, J. F.: Solitons and oscillitons in multi-ion plasmas, Nonlin. Processes. Geophys., 10, 121-130, 2003.

Spencer, R. G.: The Hamiltonian structure of multi-species fluid electrodynamics, in: "Mathematical methods in hydrodynamics and integrability in dynamical systems", edited by: Tabor, $\mathrm{M}$ and Treve, Y. M., AIP Proc. Conf., 88, 121-126, 1982.

Spencer, R. G. and Kaufman, A. N.: Hamiltonian structure of twofluid plasma dynamics, Phys. Rev. A, 25, 4, 2437-2439, 1982a.

Steeb, W. H. and Euler, N.: Nonlinear evolution equations and Painlevé test, World Scientific, Singapore, 9, 1988.

Verheest, F., Cattaert, T., Dubinin, E., Sauer, K., and McKenzie, J. F.: Whistler oscillitons revisited: the role of charge neutrality?, Nonlin. Processes. Geophys., 11, 447-452, 2004.

Webb, G. M., Axford, W. I., and Terasawa, T.: On the drift mechanism for energetic charged particles at shocks, Ap. J., 270, 537553, 1983.

Webb, G. M., McKenzie, J. F., Dubinin, E. M., and Sauer, K.: Hamiltonian formulation of nonlinear travelling whistler waves, Nonlinear Processes. Geophys., 12, 643-660, 2005.

Webb, G. M., McKenzie, J. F., Mace, R. L., Ko, C. M., and Zank, G. P.: Dual variational principles for nonlinear traveling waves in multifluid plasmas, Phys. Plasmas, 14, 082318, 1-17, 2007.

Webb, G. M., Ratkiewicz, R., Brio, M., and Zank, G. P.: Multidimensional MHD simple waves, Proc. Solar Wind 8, AIP Proc. Conf., 382, (Eds. D. Winterhalter, J. T. Gosling, S. R. Habbal, W. S. Kurth, M. Neugebauer), 335-338, 1996.

Zakharov, V. E.: Hamiltonian formalism of hydrodynamic plasma models, Sov. Phys. JETP, 33, 927-933, 1971.

Zakharov, V. E.: What is Integrability, Ed. Zakharov, V. E., v-viii pp. Springer, 1991.

Zaslavsky, G .M., Sagdeev, R. Z., Usikov, D. A., and Chernikov, A. A.: Weak Chaos and Quasi-Regular Patterns, Cambridge University Press, Cambridge, UK, 28-35, 1991. 